Supporting Information

\title{
Closely Related Benzylene-Linked Diamidophosphine Scaffolds and Their Zirconium and Hafnium Complexes: How Small Changes of the Ligand Result in Different Complex Stabilities and Reactivities
}

\author{
Sonja Batke, Malte Sietzen, Lukas Merz, Hubert Wadepohl, Joachim Ballmann*
}

Anorganisch-Chemisches Institut, Universität Heidelberg, Im Neuenheimer Feld 276, 69120 Heidelberg, Germany.

\section{Table of Contents}

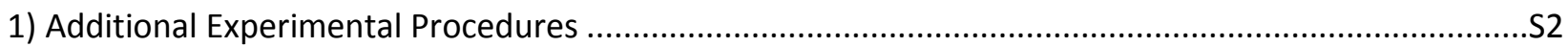

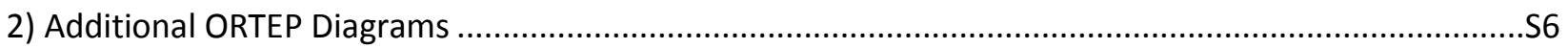

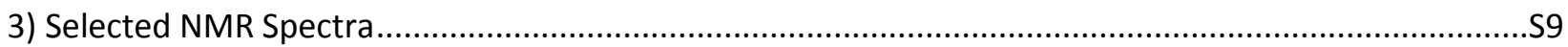

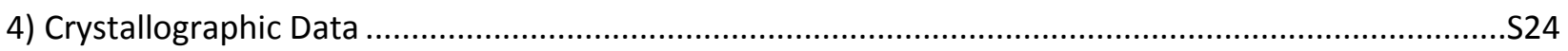

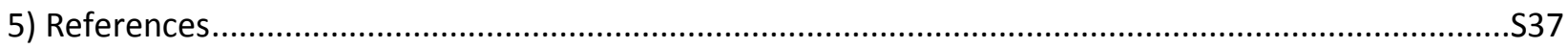




\section{1) Additional Experimental Details}

2-((dimethylamino)methyl)- $N$-phenylaniline (2). $N, N$-dimethyl-2-(phenylamino)benzamide ${ }^{1}(\mathbf{1}, 21.6 \mathrm{~g}$, $90 \mathrm{mmol}, 1.00 \mathrm{eq})$ was added in small portions to a cooled $\left(0^{\circ} \mathrm{C}\right)$, vigorously stirred suspension of $\mathrm{LiAlH}_{4}$ $(11.5 \mathrm{~g}, 302 \mathrm{mmol}, 3.20 \mathrm{eq})$ in THF ( $300 \mathrm{ml})$ within 20 minutes. The ice bath was removed and the mixture was stirred at room temperature for one hour then at $50{ }^{\circ} \mathrm{C}$ for $12 \mathrm{~h}$. The reaction mixture was chilled to $0{ }^{\circ} \mathrm{C}$ and water (approximately $40 \mathrm{ml}$ ) was added carefully until the gas evolution ceased. $\mathrm{Et}_{2} \mathrm{O}(500 \mathrm{ml})$ was added and the mixture was filtered through a plug of Celite and $\mathrm{Na}_{2} \mathrm{SO}_{4}$. The filtrate was condensed under reduced pressure and the crude product subjected to bulb to bulb distillation $\left(10^{-2} \mathrm{mbar}, \mathrm{T}_{\text {oilbath }}=\right.$ $160^{\circ} \mathrm{C}$ ). The product was obtained as a pale yellow oil, which solidified upon standing for several days at room temperature $(14.8 \mathrm{~g}, 65.5 \mathrm{mmol}, 73 \%)$. Analytical data were found in accordance with the ones previously reported. ${ }^{2}{ }^{1} \mathrm{H}$ NMR $\left(600 \mathrm{MHz}, \mathrm{C}_{6} \mathrm{D}_{6}\right): \delta[\mathrm{ppm}]=8.67(\mathrm{bs}, 1 \mathrm{H}, \mathrm{NH}), 7.50(\mathrm{~d}, J=8.1 \mathrm{~Hz}, 1 \mathrm{H}, \operatorname{ArH})$, $7.38(\mathrm{t}, J=7.7 \mathrm{~Hz}, 2 \mathrm{H}, \mathrm{ArH}), 7.30(\mathrm{dd}, J=4.6 \mathrm{~Hz}, J=12.4 \mathrm{~Hz}, 1 \mathrm{H}, \mathrm{ArH}), 7.22(\mathrm{dd}, J=8.0 \mathrm{~Hz}, J=11.7 \mathrm{~Hz}, 3 \mathrm{H}$, $\operatorname{ArH}), 7.01(\mathrm{t}, J=7.3 \mathrm{~Hz}, 1 \mathrm{H}, \mathrm{ArH}), 6.92(\mathrm{t}, J=7.4 \mathrm{~Hz}, \mathrm{ArH}, 1 \mathrm{H}), 3.58\left(\mathrm{~s}, 2 \mathrm{H}, \mathrm{ArCH}_{2}\right), 2.36\left(\mathrm{~s}, 6 \mathrm{H}, \mathrm{NMe}_{2}\right)$; Elemental analysis calcd. for $\mathrm{C}_{15} \mathrm{H}_{18} \mathrm{~N}_{2}$ : C 79.61, H 8.02, N 12.38; found C 79.65, H 7.83, N 12.31.

[2-((trimethylammonium)methyl)- $\mathbf{N}$-phenylaniline] iodide (3). To a solution of 2 (5.50 g, $24.0 \mathrm{mmol}$, $1.00 \mathrm{eq})$ in $\mathrm{Et}_{2} \mathrm{O}(80 \mathrm{ml})$, methyl iodide $(5.50 \mathrm{ml}, 88.0 \mathrm{mmol}, 3.67 \mathrm{eq})$ was added slowly and the resulting solution was refluxed for two days. Over the course of the reaction, the crude product precipitated from the yellow reaction mixture. The resulting suspension was allowed to cool to room temperature and the precipitate collected on a sinter glass frit and washed with $\mathrm{Et}_{2} \mathrm{O}(100 \mathrm{ml})$. In addition to the desired iodide salt, a considerable amount of the triiodide was formed as well. This bi-product was extracted from the crude product with boiling THF $(100 \mathrm{ml})$ to afford the desired pure iodide as a white solid (3.04 $\mathrm{g}$, $8.26 \mathrm{mmol}, 34 \%)$. Note that the iodide and triiodide cannot be distinguished by NMR spectroscopy. Each batch should be checked for the presence of the triiodide by elemental analysis or iodometric titration as high triiodide contents lead to phosphine oxidation in the next step. Nevertheless, the values obtained in a typical EA run are still lower than expected, indicative of minor amounts of the triiodide, e.g. calcd. for $\mathrm{C}_{16} \mathrm{H}_{21} \mathrm{IN}_{2}$ : C 52.18, H $5.75 \mathrm{~N} 7.61$; found C 50.35, H 5.48, N 7.18; ${ }^{1} \mathrm{H}$ NMR $\left(600 \mathrm{MHz}, \mathrm{C}_{6} \mathrm{D}_{6}\right): \delta[p p m]=7.68$ (bs, $1 \mathrm{H}, \mathrm{NH}), 7.48\left(\mathrm{dd},{ }^{4} J_{\mathrm{H}, \mathrm{H}}=1.3 \mathrm{~Hz},{ }^{3} J_{\mathrm{H}, \mathrm{H}}=7.7 \mathrm{~Hz}, 1 \mathrm{H}, 3-\mathrm{ArH}\right), 7.34\left(\mathrm{dd},{ }^{4} J_{\mathrm{H}, \mathrm{H}}=0.9 \mathrm{~Hz},{ }^{3} J_{\mathrm{H}, \mathrm{H}}=8.3 \mathrm{~Hz}, 1 \mathrm{H}, 6-\right.$ ArH), $7.29-7.25(\mathrm{~m}, 1 \mathrm{H}, 5-\mathrm{ArH}), 7.24\left(\mathrm{dd},{ }^{4} \mathrm{~J}_{\mathrm{H}, \mathrm{H}}==1.0 \mathrm{~Hz},{ }^{3} J_{\mathrm{H}, \mathrm{H}}=8.5 \mathrm{~Hz}, 2 \mathrm{H}, o-\mathrm{NPh}\right), 7.22-7.17(\mathrm{~m}, 2 \mathrm{H}, m-$ $\mathrm{NPh}), 6.91-6.90(\mathrm{~m}, 1 \mathrm{H}, 4-\mathrm{Ar}-\mathrm{H}), 6.88\left(\mathrm{~d},{ }^{3} \mathrm{H}_{\mathrm{H}, \mathrm{H}}=7.4 \mathrm{~Hz}, 1 \mathrm{H}, p-\mathrm{NPh}\right), 5.35\left(\mathrm{~s}, 2 \mathrm{H}, \operatorname{ArCH}_{2}\right), 3.26(\mathrm{~s}, 9 \mathrm{H}$, $\left.\mathrm{NMe}_{3}\right) ;{ }^{13} \mathrm{C}\left\{{ }^{1} \mathrm{H}\right\}$ NMR $\left(151 \mathrm{MHz}, \mathrm{C}_{6} \mathrm{D}_{6}\right): \delta$ [ppm] = 145.3 (s, 1-ArC), 142.7 (s, ipso-NPh), 135.8 (s, 3-ArC), 132.2 (s, 5-ArC), 129.4 (s, m-NPh), 121.7 (s 4-ArC), 121.5 (s, p-NPh), 120.3 (s, 6-ArC), 118.7 (s, m-NPh), 117.0 (s, 1-ArC), 65.2 (s, $\mathrm{ArCH}_{2}$ ), 53.1 (s, $\mathrm{NMe}_{3}$ ); HR-MS (FAB ${ }^{+}$): observed $m / z=241.1721$ (94), calcd. $m / z$ for $\mathrm{C}_{16} \mathrm{H}_{21} \mathrm{~N}_{2}$ $\left([\mathrm{M}-\mathrm{I}]^{+}\right)=241.1699$, observed $m / z=182.0979(100)$, calcd. $m / z$ for $\mathrm{C}_{13} \mathrm{H}_{12} \mathrm{~N}\left(\left[\mathrm{M}-\mathrm{I}-\mathrm{NMe}_{3}\right]^{+}\right)=182.0970$.

$\mathbf{N}$-(2-bromobenzyl)-2,6-dimethylaniline (6). A stirred solution of 2-bromobenzaldehyde $(50 \mathrm{~g}, 262 \mathrm{mmol}$, $1.0 \mathrm{eq})$ and 3,5 dimethylaniline $(32 \mathrm{~g}, 33 \mathrm{ml}, 262 \mathrm{mmol}, 1.0 \mathrm{eq})$ in methanol $(300 \mathrm{ml})$ was heated to $90{ }^{\circ} \mathrm{C}$ for $2.5 \mathrm{~h}$. After the reaction mixture was allowed to cool to room temperature, sodium borohydride (13.7 g, $362 \mathrm{mmol}, 1.4 \mathrm{eq})$ was added and stirring continued at room temperature overnight. The reaction mixture was then heated to $70^{\circ} \mathrm{C}$ for $8 \mathrm{~h}$ and subsequently allowed to cool to room temperature. After all volatiles were removed, the residue was dissolved in toluene $(200 \mathrm{ml})$, washed with water $(100 \mathrm{ml})$ and the aqueous phase was extracted with toluene $(2 \times 100 \mathrm{ml})$. The combined organic phases were dried over $\mathrm{Na}_{2} \mathrm{SO}_{4}$ and concentrated under reduced pressure to afford the product as a viscous oil $(72 \mathrm{~g}, 247 \mathrm{mmol}$, 
$94 \%) .{ }^{1} \mathrm{H}$ NMR $\left(600 \mathrm{MHz}, \mathrm{C}_{6} \mathrm{D}_{6}\right): \delta[\mathrm{ppm}]=7.36\left(\mathrm{dd},{ }^{3} \mathrm{~J}_{\mathrm{H}, \mathrm{H}}=7.9 \mathrm{~Hz},{ }^{4} J_{\mathrm{H}, \mathrm{H}}=1.1 \mathrm{~Hz}, 1 \mathrm{H}, 6-\mathrm{ArH}\right), 7.23-7.20$ $(\mathrm{m}, 1 \mathrm{H}, 3-\mathrm{ArH}), 6.86\left(\mathrm{td},{ }^{3} \mathrm{H,H}=7.6 \mathrm{~Hz},{ }^{4} J_{\mathrm{H}, \mathrm{H}}=1.0 \mathrm{~Hz}, 1 \mathrm{H}, 5-\mathrm{ArH}\right), 6.67\left(\mathrm{td},{ }^{3} \mathrm{~J}_{\mathrm{H}, \mathrm{H}}=7.9 \mathrm{~Hz},{ }^{4} J_{\mathrm{H}, \mathrm{H}}=1.7 \mathrm{~Hz}, 1 \mathrm{H}\right.$, 4-ArH), 6.38 (s, $1 \mathrm{H}, p-\mathrm{Xyl}), 6.13(\mathrm{~s}, 2 \mathrm{H}, \mathrm{o}-\mathrm{Xyl}), 4.17\left(\mathrm{~s}, 2 \mathrm{H}, \mathrm{CH}_{2}\right), 3.41(\mathrm{~s}, 1 \mathrm{H}, \mathrm{NH}), 2.12\left(\mathrm{~s}, 6 \mathrm{H}, \mathrm{Xyl}-\mathrm{CH}_{3}\right)$; ${ }^{13} \mathrm{C}\left\{{ }^{1} \mathrm{H}\right\}$ NMR $\left(101 \mathrm{MHz}, \mathrm{C}_{6} \mathrm{D}_{6}\right): \delta[\mathrm{ppm}]=148.2$ (s, ipso-Xyl), 139.2 (s, m-Xyl), 138.8 (s, 1-ArC), 132.9 (s, 6$\operatorname{ArC}$ ), 129.3 (s, 3-ArC), 128.3 (s, 4-ArC), 127.7 (s, 5-ArC), 123.4 (s, 2-ArC), 120.2 (s, p-Xyl), 111.2 (s, o-Xyl), $48.5\left(\mathrm{~s}, \mathrm{CH}_{2}\right), 21.7\left(\mathrm{~s}, \mathrm{Xyl}-\mathrm{CH}_{3}\right)$; HR-MS (DART ${ }^{+}$): observed $\mathrm{m} / \mathrm{z}=290.05383$ and $292.05174\left(\mathrm{M}+\mathrm{H}^{+}\right.$for ${ }^{79} \mathrm{Br}$ and $\left.{ }^{81} \mathrm{Br}\right)$, calcd. $m / z=290.05389$ and $292.05184\left(\mathrm{C}_{15} \mathrm{H}_{16} \mathrm{~N}^{79} \mathrm{Br}+\mathrm{H}^{+}\right.$and $\left.\mathrm{C}_{15} \mathrm{H}_{16} \mathrm{~N}^{81} \mathrm{Br}+\mathrm{H}^{+}\right)$.

In the main text, it is mentioned that the $\left[{ }^{\mathrm{Ph}} \mathbf{A}\right]$ - and $[\mathbf{B}]$-coordinated titanium diiodides $\left[{ }^{\mathrm{Ph}} \mathbf{A}\right] \mathrm{Til}_{2}$ and $[\mathbf{B}] \mathrm{Til}_{2}$ have been prepared via the corresponding bis-dimethylamides $\left[{ }^{\mathrm{Ph}} \mathbf{A}\right]-\mathbf{1}-\mathrm{Ti}$ and $[\mathbf{B}]-\mathbf{1}-\mathbf{T i}$ and that a similar approach met with failure in the case of the $N, N^{\prime}$-bis(trimethylsilyl)-substituted ligand $\left[{ }^{5 i} A\right] H_{2}$. This observation is in line with our previous report on related $N, N^{\prime}, N^{\prime \prime}$-tris(trimethylsilyl)-substituted triamidophosphine ligands and their reactivity towards titanium: ${ }^{3}$ silyl amide bond cleavage seems to represent a major drawback in these silyl-substituted systems. Although the titanium complexes [ $\left.{ }^{\mathrm{Ph}} \mathbf{A}\right]-\mathbf{2}-$ $\mathrm{Ti}$ and [B]-2-Ti could be obtained, both species failed to react cleanly with alkylating reagents (PhLi, $\mathrm{Bn}_{2} \mathrm{Mg}(\text { thf })_{2}$ or $\mathrm{Me}_{3} \mathrm{SiCH}_{2} \mathrm{Li}$ ). In all cases, dark reaction mixtures were obtained, which contained reduced species (as judged by EPR spectroscopy) as well as NMR-active unidentified diamagnetic products. So far, these product mixtures could not be separated. The experimental procedures for the preparation of [B]1-Ti, [B]-2-Ti, [ $\left.{ }^{\mathrm{Ph}} \mathrm{A}\right]-\mathbf{1}-\mathrm{Ti}$ and $\left[{ }^{\mathrm{Ph}} \mathrm{A}\right]-\mathbf{2}-\mathrm{Ti}$ are provided below.

[B]Ti(NMe $)_{2}$ ([B]-1-Ti). A solution of Ti(NMe $)_{4}(233 \mathrm{mg}, 10.4 \mathrm{mmol}, 1.10 \mathrm{eq})$ in toluene $(5 \mathrm{ml})$ was added to a stirred solution of $[B] \mathrm{H}_{2}(500 \mathrm{mg}, 9.46 \mathrm{mmol}, 1.00 \mathrm{eq})$ in toluene $(10 \mathrm{ml})$ and the reaction mixture was heated to $110^{\circ} \mathrm{C}$ for $12 \mathrm{~h}$. After cooling to room temperature, all volatiles were removed under reduced pressure and the residue was washed with $n$-pentane $(2 \times 2 \mathrm{~mL})$. After drying in vacuum, the product was obtained as a dark red powder (558 mg, $8.42 \mathrm{mmol}, 89 \%) .{ }^{1} \mathrm{H} \mathrm{NMR}\left(600 \mathrm{MHz}, \mathrm{C}_{6} \mathrm{D}_{6}\right): \delta[\mathrm{ppm}]=7.23-7.18$

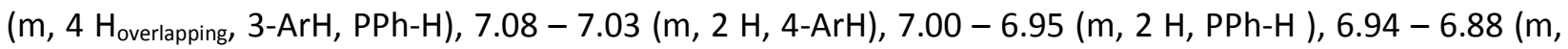
$\left.3 \mathrm{H}_{\text {overlapping, }} 6-\mathrm{ArH}, \mathrm{PPh}-\mathrm{H}\right), 6.80$ (td, $\left.J=1.0 \mathrm{~Hz}, J=7.5,2 \mathrm{H}, 5-\mathrm{ArH}\right), 6.65(\mathrm{~s}, 4 \mathrm{H}, o-\mathrm{Xyl}), 6.46$ (s, $2 \mathrm{H}, p$-Xyl), $5.41\left(\mathrm{~d}, J=15.5 \mathrm{~Hz}, 2 \mathrm{H}, \mathrm{CH}_{2}\right), 4.91\left(\mathrm{dd}, J=15.5 \mathrm{~Hz}, J=1.4 \mathrm{~Hz}, 2 \mathrm{H}, \mathrm{CH}_{2}\right), 3.27\left(\mathrm{~s}, 6 \mathrm{H}, \mathrm{NMe}_{2}\right), 3.18(\mathrm{~s}, 6 \mathrm{H}$, $\mathrm{NMe}_{2}$ ), 2.25 (s, $\left.12 \mathrm{H}, \mathrm{Xyl}-\mathrm{CH}_{3}\right) ;{ }^{13} \mathrm{C}\left\{{ }^{1} \mathrm{H}\right\} \operatorname{NMR}\left(151 \mathrm{MHz}, \mathrm{C}_{6} \mathrm{D}_{6}\right): \delta$ [ppm] = 154.3 (s, ipso-Xyl), 147.3 (d, $J=20.3 \mathrm{~Hz}, 1-\operatorname{ArC}$ ), 137.2 (s, $m-X y l), 136.6$ (d, $J=4.9 \mathrm{~Hz}, 2-\operatorname{ArC}), 135.4$ (d, J=2.9 Hz, 6-ArC), 132.7 (d, $J=14.0 \mathrm{~Hz}, \mathrm{PPh}-\mathrm{C}), 129.6$ (d, J = 8.1 Hz, 3-ArC), 129.4 (s, PPh-C), 128.1 (s, 4-ArC), 128.1 (s, PPh-C), 128.0 (d, $J=3.2 \mathrm{~Hz}, \mathrm{PPh}-\mathrm{C}$ ), 127.2 (d, J=2.7 Hz, 5-ArC), 120.8 (s, p-Xyl), 115.6 (s,o-Xyl), 53.2 (d, J=14.5 Hz, CH CH $48.3\left(\mathrm{~d}, J=5.6 \mathrm{~Hz}, \mathrm{NMe}_{2}\right), 46.5\left(\mathrm{~s}, \mathrm{NMe}_{2}\right), 21.7\left(\mathrm{~s}, \mathrm{Xyl}-\mathrm{CH}_{3}\right) ;{ }^{31} \mathrm{P}\left\{{ }^{1} \mathrm{H}\right\} \mathrm{NMR}\left(243 \mathrm{MHz}, \mathrm{C}_{6} \mathrm{D}_{6}\right): \delta[\mathrm{ppm}]=-29.3$ (s); Elemental analysis calcd. for $\mathrm{C}_{40} \mathrm{H}_{47} \mathrm{~N}_{4} \mathrm{PTT}$ : C 72.50, H 7.15, N 8.45; found: C 72.77, H 7.68, N 8.06.

[B]Til 2 ([B]-2-Ti). A solution of trimethylsilyl iodide $(0.17 \mathrm{ml}, 254 \mathrm{mg}, 1.27 \mathrm{mmol}, 2.10 \mathrm{eq})$ in toluene (5 mL) was added dropwise to a stirred solution of $16-\mathrm{Ti}(400 \mathrm{mg}, 0.60 \mathrm{mmol}, 1.00 \mathrm{eq})$ in toluene $(15 \mathrm{~mL})$ and the resulting reaction mixture was stirred for $30 \mathrm{~min}$ at room temperature. All volatiles were removed in 
vacuum and the residue was washed with a mixture (50 vol-\% each) of diethylether and pentane ( $2 \times 3 \mathrm{ml})$. After drying in vacuum, the title compound was obtained as a maroon solid (413 $\mathrm{mg}, 0.50 \mathrm{mmol}, 83 \%$ ).

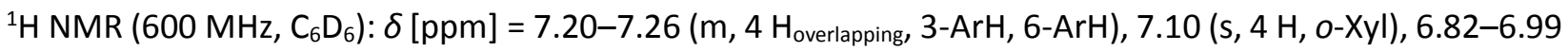

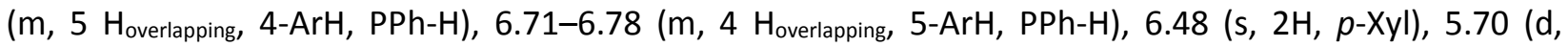
$\left.J=17.5 \mathrm{~Hz}, 2 \mathrm{H}, \mathrm{CH}_{2}\right), 4.76\left(\mathrm{~d}, J=17.5 \mathrm{~Hz}, 2 \mathrm{H}, \mathrm{CH}_{2}\right), 2.01\left(\mathrm{~s}, 12 \mathrm{H}, \mathrm{Xyl}-\mathrm{CH}_{3}\right) ;{ }^{13} \mathrm{C}\left\{{ }^{1} \mathrm{H}\right\} \mathrm{NMR}\left(151 \mathrm{MHz}, \mathrm{C}_{6} \mathrm{D}_{6}\right)$ : $\delta$ [ppm] = 158.2 (s, ipso-Xyl), 149.4 (d, $J=20.1 \mathrm{~Hz}, 1-\operatorname{ArC}), 141.7$ (s, $m$-Xyl), 133.1 (d, $J=2.4 \mathrm{~Hz}, 6-\operatorname{ArC}), 132.8$ (d, $J=9.5 \mathrm{~Hz}, \mathrm{PPh}-\mathrm{C}$ ), 130.4 (d, $J=7.6 \mathrm{~Hz}, 2-\operatorname{ArC}), 129.6$ (d, $J=1.8 \mathrm{~Hz}, 4-\operatorname{ArC}$ ), 128.6 (d, $J=2.1 \mathrm{~Hz}, 5-\operatorname{ArC}$ ), 127.4 (d, J = 9.2 Hz, 3-ArC), 126.8 (s, PPh-C), 126.4 (d, J = 13.2 Hz, PPh-C), 126.3 (d, J = 8.7 Hz, PPh-C), 126.1 (s, $p$-Xyl), $116.1(\mathrm{~s}, o-X y l), 52.2\left(\mathrm{~d}, J=8.8 \mathrm{~Hz}, \mathrm{CH}_{2}\right), 19.8\left(\mathrm{~s}, \mathrm{Xyl}-\mathrm{CH}_{3}\right) ;{ }^{1}{ }^{1}\left\{{ }^{1} \mathrm{H}\right\} \mathrm{NMR}\left(243 \mathrm{MHz}, \mathrm{C}_{6} \mathrm{D}_{6}\right): \delta$ [ppm] = 3.0 (s); Elemental analysis calcd. for $\mathrm{C}_{36} \mathrm{H}_{35} \mathrm{I}_{2} \mathrm{~N}_{2} \mathrm{PTi}$ : C 52.20, H 4.26, N 3.38; found: $\mathrm{C} 51.86, \mathrm{H} 4.15$, N 3.36 .

$\left[{ }^{\mathrm{Ph}} \mathrm{A}\right] \mathrm{Ti}\left(\mathrm{NMe}_{2}\right)_{2}\left(\left[{ }^{\mathrm{Ph}} \mathrm{A}\right]-1-\mathrm{Ti}\right)$. A solution of $\mathrm{Ti}\left(\mathrm{NMe}_{2}\right)_{4}(124 \mathrm{mg}, 555 \mu \mathrm{mol}, 1.04 \mathrm{eq})$ in toluene (10 ml) was added slowly to a stirred solution of $\left[{ }^{\mathrm{Ph}} \mathrm{A}\right] \mathrm{H}_{2}(250 \mathrm{mg}, 530 \mu \mathrm{mol}, 1.00 \mathrm{eq})$ in toluene $(10 \mathrm{ml})$. An immediately color change to red was observed and the solution was stirred for $7 \mathrm{~d}$ at $110^{\circ} \mathrm{C}$. Subsequently, all volatiles were removed in vacuum and the residue washed with $\mathrm{Et}_{2} \mathrm{O}(2 \times 5 \mathrm{ml})$. After recrystallization from toluene at $-40{ }^{\circ} \mathrm{C}$, the product was isolated as a red crystalline solid $(73.9 \mathrm{mg}, 122 \mu \mathrm{mol}, 23 \%) .{ }^{1} \mathrm{H}$ $\operatorname{NMR}\left(400 \mathrm{MHz}\right.$, Tol- $\left.\mathrm{d}_{8}\right): \delta[\mathrm{ppm}]=7.31\left(\mathrm{~d},{ }^{3} \mathrm{~J}_{\mathrm{H}, \mathrm{H}}=7.5 \mathrm{~Hz}, 2 \mathrm{H}, \mathrm{ArH}\right), 7.22\left(\mathrm{t},{ }^{3} \mathrm{~J}_{\mathrm{H}, \mathrm{H}}=7.1 \mathrm{~Hz}, 2 \mathrm{H}, \operatorname{ArH}\right), 7.16-$ $7.10(\mathrm{~m}, 2 \mathrm{H}, \mathrm{ArH}), 7.00-6.90(\mathrm{~m}, 9 \mathrm{H}, \mathrm{ArH}), 6.83\left(\mathrm{t},{ }^{3} \mathrm{~J}_{\mathrm{H}, \mathrm{H}}=7.1 \mathrm{~Hz}, 2 \mathrm{H}, \mathrm{ArH}\right), 6.62\left(\mathrm{t},{ }^{3} \mathrm{~J}_{\mathrm{H}, \mathrm{H}}=6.7 \mathrm{~Hz}, 2 \mathrm{H}, \operatorname{ArH}\right)$, $6.38\left(\mathrm{~d},{ }^{3} \mathrm{~J}_{\mathrm{H}, \mathrm{H}}=7.1 \mathrm{~Hz}, 4 \mathrm{H}, \mathrm{ArH}\right), 3.51\left(\mathrm{~s}, 6 \mathrm{H}, \mathrm{NMe}_{2}\right), 2.89-2.75\left(\mathrm{~m}, 8 \mathrm{H}_{\text {overlapping }} \mathrm{NMe}_{2}, \mathrm{PCH}_{2}\right), 2.52$ (dd, JP,H/H,H $\left.=8.5 \mathrm{~Hz}, J_{\mathrm{P}, \mathrm{H} / \mathrm{H}, \mathrm{H}}=13.5 \mathrm{~Hz}, 2 \mathrm{H}, \mathrm{PCH}_{2}\right) ;{ }^{13} \mathrm{C}$ NMR $\left(101 \mathrm{MHz}, \mathrm{Tol}_{-} \mathrm{d}_{8}\right): \delta[\mathrm{ppm}]=149.9(\mathrm{~s}, \operatorname{ArC}), 145.1(\mathrm{~s}, \operatorname{ArC})$, $132.0(\mathrm{~d}, J=2.0 \mathrm{~Hz}, \operatorname{ArC}$ ), 131.1 (s, $\operatorname{ArC}), 131.0$ (s, $\operatorname{ArC}$ ), 131.0 (d, J = 3.9 Hz, ArC), 129.4 (s, ArC), 129.3 (s, $\operatorname{ArC}), 129.0(\mathrm{~s}, \operatorname{ArC}), 129.0(\mathrm{~d}, J=2.1 \mathrm{~Hz}, \operatorname{ArC}), 127.3(\mathrm{~s}, \operatorname{ArC}) 126.5(\mathrm{~s}, \operatorname{ArC}), 118.5(\mathrm{~s}, \operatorname{ArC}), 115.8(\mathrm{~s}, \operatorname{ArC})$, 50.2 (s, $\mathrm{NMe}_{2}$ ), 48.1 (s, NMe $)$ ), 31.5 - $31.4\left(\mathrm{~m}, \mathrm{PCH}_{2}\right) ;{ }^{31} \mathrm{P} \mathrm{NMR}\left(162 \mathrm{MHz}, \mathrm{C}_{6} \mathrm{D}_{6}\right): \delta[\mathrm{ppm}]=10.1$ (s) Elemental analysis calcd. for $\mathrm{C}_{36} \mathrm{H}_{39} \mathrm{~N}_{4} \mathrm{PTi}$ : C 71.28, H $6.48 \mathrm{~N} 9.24$; found $\mathrm{C} 70.84, \mathrm{H} 6.48 \mathrm{~N} 9.34$. Note that the carbon value is not within the expected $\pm 0.4 \%$ range. As the compound appears to be pure by NMR spectroscopy, this difference might either be due to minor amounts of paramagnetic impurities ( $\mathrm{Ti}^{\mathrm{III}}$ ) or due to carbide formation upon combustion.

$\left[{ }^{\mathrm{Ph}} \mathrm{A}\right] \mathrm{Til}_{2}\left(\left[{ }^{\mathrm{Ph}} \mathrm{A}\right]-2-\mathrm{Ti}\right)$. Neat trimethylsilyl iodide $(17.3 \mathrm{mg}, 86.5 \mu \mathrm{mol}, 2.1 \mathrm{eq})$ was added dropwise to a solution of $\left[{ }^{\mathrm{Ph}} \mathrm{A}\right]-\mathbf{1}-\mathrm{Ti}(25.0 \mathrm{mg}, 41.2 \mu \mathrm{mol}, 1.00 \mathrm{eq})$ in toluene $(0.5 \mathrm{ml})$ and the reaction mixture left standing without stirring for $2 \mathrm{~d}$. The deep red crystals, which formed over this period were collected on a sinter glass frit, washed with $\mathrm{Et}_{2} \mathrm{O}(2 \mathrm{ml})$ and dried in vacuum to afford the title compound $(7.00 \mathrm{mg}$, $9.06 \mu \mathrm{mol})$ in $22 \%$ yield. ${ }^{1} \mathrm{H} \mathrm{NMR}\left(400 \mathrm{MHz}, \mathrm{CD}_{2} \mathrm{Cl}_{2}\right): \delta$ [ppm] = $7.46-7.34(\mathrm{~m}, 4 \mathrm{H}, \mathrm{ArH}), 7.33-7.31(\mathrm{~m}$, $2 \mathrm{H} \mathrm{ArH},), 7.29-7.21(\mathrm{~m}, 6 \mathrm{H}, \mathrm{ArH}), 7.20-7.12(\mathrm{~m}, 5 \mathrm{H}, \mathrm{ArH}), 7.07-6.98(\mathrm{~m}, 6 \mathrm{H}, \mathrm{ArH}), 3.32\left(\mathrm{dd}, J_{\mathrm{P}, \mathrm{H} / \mathrm{H}, \mathrm{H}}=\right.$ $\left.9.9 \mathrm{~Hz}, J_{\mathrm{P}, \mathrm{H} / \mathrm{H}, \mathrm{H}}=14.4 \mathrm{~Hz}, 2 \mathrm{H}, \mathrm{PCH}_{2}\right), 3.09\left(\mathrm{dd}, J_{\mathrm{P}, \mathrm{H} / \mathrm{H}, \mathrm{H}}=12.0 \mathrm{~Hz}, J_{\mathrm{P}, \mathrm{H} / \mathrm{H}, \mathrm{H}}=14.3 \mathrm{~Hz}, 2 \mathrm{H}, \mathrm{PCH}_{2}\right) ;{ }^{13} \mathrm{C} \mathrm{NMR}$ $\left(101 \mathrm{MHz}, \mathrm{CD}_{2} \mathrm{Cl}_{2}\right.$ ): $\delta[\mathrm{ppm}]=154.0(\mathrm{~d}, J=2.6 \mathrm{~Hz}, \operatorname{ArC}), 139.2$ (d, J = 7.9 Hz, ArC), $133.0(\mathrm{~s}, \operatorname{ArC}), 131.7(\mathrm{~d}, J$ = 22.4 Hz, ArC), 131.2 (s, ArC), 131.1 (s, ArC), 130.7 (d, J = 4.4 Hz, ArC), 130.4 (d, J = 5.8 Hz, ArC), 130.1 (d, $J=2.3 \mathrm{~Hz}, \operatorname{ArC}$ ), 129.9 (s, ArC), 129.1 (d, J = 8.8 Hz, ArC), 128.9 (s, ArC), 125.0 (s, ArC), 118.5 (s, ArC), 29.3 $\left(\mathrm{d}, J=10.9 \mathrm{~Hz}, \mathrm{PCH}_{2}\right) ;{ }^{31} \mathrm{P} \mathrm{NMR}\left(162 \mathrm{MHz}, \mathrm{CD}_{2} \mathrm{Cl}_{2}\right): \delta$ [ppm] = 2.15 (s); Elemental analysis calcd. for $\mathrm{C}_{32} \mathrm{H}_{27} \mathrm{I}_{2} \mathrm{~N}_{2}$ PTi: C 49.77, H 3.52, N 3.63; found C 49.06, H 3.74, N 3.43. Note that the carbon value is not within the expected $\pm 0.4 \%$ range. As the compound appears to be pure by NMR spectroscopy, this difference might either be due to minor amounts of paramagnetic impurities ( $\mathrm{Ti}^{\mathrm{III}}$ ) or due to carbide formation upon combustion. 
In case of $\left[{ }^{\mathrm{Ph}} \mathbf{A}\right] \mathrm{H}_{2}$, the zirconium and hafnium dichloro complexes $\left[{ }^{\mathrm{Ph}} \mathbf{A}\right] \mathrm{ZrCl}_{2}$ and $\left[{ }^{\mathrm{Ph}} \mathbf{A}\right] \mathrm{HfCl}_{2}\left(\mathrm{OEt} \mathrm{H}_{2}\right)$ were prepared via protonolysis of $\left(\mathrm{Me}_{3} \mathrm{SiCH}_{2}\right)_{2} \mathrm{ZrCl}_{2}\left(\mathrm{OEt}_{2}\right)_{2}$ and $\mathrm{Bn}_{2} \mathrm{HfCl}_{2}\left(\mathrm{OEt}_{2}\right)$, respectively. In comparison to the diiodides $\left[{ }^{\mathrm{Ph}} \mathrm{A}\right]-\mathbf{2}-\mathbf{Z r}$ and $\left[{ }^{\mathrm{Ph}} \mathrm{A}\right]-\mathbf{2}-\mathrm{Hf}$, identical reactivities were found for both chlorides. Given that the preparation of the starting materials $\left(\mathrm{Me}_{3} \mathrm{SiCH}_{2}\right)_{2} \mathrm{ZrCl}_{2}\left(\mathrm{OEt}_{2}\right)_{2}$ and $\mathrm{Bn}_{2} \mathrm{HfCl}_{2}\left(\mathrm{OEt}_{2}\right)$ is less convenient in comparison to the use of $\mathrm{Zr}\left(\mathrm{NMe}_{2}\right)_{4}$ and $\mathrm{Hf}\left(\mathrm{NMe}_{2}\right)_{4}$, followed by treatment with $\mathrm{Me}_{3} \mathrm{Sil}$, these compounds have not been employed routinely. The zirconium derivative could not be obtained in an analytically pure form (c.f. elemental analysis of $\left[{ }^{\mathrm{Ph}} \mathrm{A}\right] \mathrm{ZrCl}_{2}$ ). Experimental procedures for the preparation of $\left[{ }^{\mathrm{Ph}} \mathrm{A}\right] \mathrm{ZrCl}_{2}$ and $\left[{ }^{\mathrm{Ph}} \mathrm{A}\right] \mathrm{HfCl}_{2}\left(\mathrm{OEt}_{2}\right)$ are provided below.

[ $\left.{ }^{\mathrm{Ph}} \mathrm{A}\right] \mathrm{ZrCl}_{2}$. A solution of $\left(\mathrm{Me}_{3} \mathrm{SiCH}_{2}\right)_{2} \mathrm{ZrCl}_{2}\left(\mathrm{OEt}_{2}\right)_{2}(268 \mathrm{mg}, 555 \mu \mathrm{mol}, 1.05 \mathrm{eq}$.) in toluene $(10 \mathrm{ml})$ was added slowly to a stirred solution of [ $\left.{ }^{\mathrm{Ph}} \mathrm{A}\right] \mathrm{H}_{2}(250 \mathrm{mg}, 530 \mu \mathrm{mol}, 1.00 \mathrm{eq}$.) in toluene $(10 \mathrm{ml})$. An immediately color change to yellow was observed and the solution was stirred $5 \mathrm{~h}$ at $70^{\circ} \mathrm{C}$. Subsequently, all volatiles were removed in vacuum and the residue washed with $\mathrm{Et}_{2} \mathrm{O}(2 \times 5 \mathrm{ml})$. The product was obtained as a pale yellow solid (143 mg, $226 \mu \mathrm{mol}, 43 \%) .{ }^{1} \mathrm{H}$ NMR $\left(600 \mathrm{MHz}, \mathrm{CD}_{2} \mathrm{Cl}_{2}\right): \delta[\mathrm{ppm}]=7.47-7.40(\mathrm{~m}, 2 \mathrm{H}, \mathrm{ArH}), 7.38$ $\left(d,{ }^{3} J_{H, H}=5.9 \mathrm{~Hz}, 1 \mathrm{H}, \mathrm{ArH}\right), 7.33\left(\mathrm{t},{ }^{3} \mathrm{~J}_{\mathrm{H}, \mathrm{H}}=7.2 \mathrm{~Hz}, 2 \mathrm{H}, \mathrm{ArH}\right), 7.18\left(\mathrm{dd},{ }^{3} J_{\mathrm{H}, \mathrm{H}}=8.2 \mathrm{~Hz},{ }^{3} J_{\mathrm{H}, \mathrm{H}}=15.9 \mathrm{~Hz}, 4 \mathrm{H}, \mathrm{ArH}\right)$, $7.12-7.06(\mathrm{~m}, 4 \mathrm{H}, m-\mathrm{NPh}), 7.09-6.98(\mathrm{~m}, 4 \mathrm{H}, \mathrm{ArH}), 6.75\left(\mathrm{t}, 3^{3} \mathrm{H}_{\mathrm{H}}=7.3 \mathrm{~Hz}, 2 \mathrm{H}, p-\mathrm{NPh}\right), 6.59\left(\mathrm{~d}, 3^{3} \mathrm{H}_{\mathrm{H}}=\right.$ $8.0 \mathrm{~Hz}, 4 \mathrm{H}, o-\mathrm{NPh}), 3.05-3.00\left(\mathrm{~m}, 4 \mathrm{H}, \mathrm{PCH}_{2}\right) ;{ }^{13} \mathrm{C}\left\{{ }^{1} \mathrm{H}\right\} \mathrm{NMR}\left(151 \mathrm{MHz}, \mathrm{CD}_{2} \mathrm{Cl}_{2}\right): \delta[\mathrm{ppm}]=150.9(\mathrm{~s}, \operatorname{ArC})$, $138.7\left(\mathrm{~d}, J_{\mathrm{P}, \mathrm{C}}=7.6 \mathrm{~Hz}, \operatorname{ArC}\right.$ ), 136.5 (s, ArC), $133.3(\mathrm{~s}, \operatorname{ArC}), 132.4\left(\mathrm{~d}, J_{\mathrm{P}, \mathrm{C}}=11.2 \mathrm{~Hz}, \operatorname{ArC}\right), 131.73(\mathrm{~s}, \operatorname{ArC}), 131.6$ $\left(d, J_{P, C}=5.2 \mathrm{~Hz}, \operatorname{ArC}\right), 130.6\left(\mathrm{~d}, J_{\mathrm{P}, \mathrm{C}}=2.6 \mathrm{~Hz}, \operatorname{ArC}\right), 129.5(\mathrm{~s}, m-\mathrm{NPh}), 129.2(\mathrm{~s}, \operatorname{ArC}), 128.7(\mathrm{~s}, \operatorname{ArC}), 125.8(\mathrm{~s}$, $\operatorname{ArC}$ ), 122.1 (s, $p$-NPh), 117.5 (s, m-NPh), $30.1\left(\mathrm{~d},{ }^{1} \mathrm{~J}_{\mathrm{P}, \mathrm{C}}=9.8 \mathrm{~Hz}, \mathrm{PCH}_{2}\right) ;{ }^{31} \mathrm{P}\left\{{ }^{1} \mathrm{H}\right\} \mathrm{NMR}\left(243 \mathrm{MHz}, \mathrm{CD}_{2} \mathrm{Cl}_{2}\right): \delta$ [ppm] = 8.0 (s); In two attempts, no fitting elemental analysis was obtained (e.g. calcd. for $\mathrm{C}_{32} \mathrm{H}_{27} \mathrm{Cl}_{2} \mathrm{~N}_{2} \mathrm{PZr}$ : C 60.75, H 4.30, N 4.43; found C 59.61, H 5.02, N 3.90), not even for recrystallized samples (from toluene/ $\mathrm{Et}_{2} \mathrm{O}$ at $-40^{\circ} \mathrm{C}$ ). No further attempts were made in view of the above stated more convenient preparation of the corresponding diiodide $\left[{ }^{\mathrm{Ph}} \mathrm{A}\right]-\mathbf{2}-\mathrm{Zr}$.

$\left[{ }^{\mathrm{Ph}} \mathrm{A}\right] \mathrm{HfCl}_{2}\left(\mathrm{OEt}_{2}\right)$ A solution of $\mathrm{Bn}_{2} \mathrm{HfCl}_{2}\left(\mathrm{OEt}_{2}\right)(320 \mathrm{mg}, 556 \mu \mathrm{mol}, 1.05$ eq.) in toluene $(10 \mathrm{ml})$ was added slowly to a stirred solution of $\left[{ }^{\mathrm{Ph}} \mathrm{A}\right] \mathrm{H}_{2}(250 \mathrm{mg}, 530 \mu \mathrm{mol}, 1.00$ eq.) in toluene $(10 \mathrm{ml})$. An immediately color change to yellow was observed and the solution was stirred $24 \mathrm{~h}$ at room temperature. Subsequently, all volatiles were removed in vacuum and the residue washed with $\mathrm{Et}_{2} \mathrm{O}(2 \times 5 \mathrm{ml})$. The product was obtained as a pale yellow solid $(181 \mathrm{mg}, 228 \mu \mathrm{mol}, 43 \%) .{ }^{1} \mathrm{H} \mathrm{NMR}\left(600 \mathrm{MHz}, \mathrm{CD}_{2} \mathrm{Cl}_{2}\right): \delta[\mathrm{ppm}]=7.52-7.38(\mathrm{~m}, 5 \mathrm{H}$, ArH), $7.32-7.23(\mathrm{~m}, 4 \mathrm{H}, \mathrm{ArH}), 7.20-7.13(\mathrm{~m}, 4 \mathrm{H}, \mathrm{ArH}), 7.10\left(\mathrm{dd},{ }^{3} J_{\mathrm{H}, \mathrm{H}}=7.4 \mathrm{~Hz},{ }^{3} \mathrm{~J}_{\mathrm{H}, \mathrm{H}}=8.4 \mathrm{~Hz}, 4 \mathrm{H}, m-\mathrm{NPh}\right)$, $6.78\left(\mathrm{t}, 3^{3} \mathrm{~J}_{\mathrm{H}, \mathrm{H}}=7.3 \mathrm{~Hz}, 2 \mathrm{H}, p-\mathrm{NPh}\right), 6.66\left(\mathrm{~d},{ }^{3} \mathrm{~J}_{\mathrm{H}, \mathrm{H}}=7.8 \mathrm{~Hz}, 4 \mathrm{H}, \mathrm{o}-\mathrm{NPh}\right), 3.45\left(\mathrm{q},{ }^{3} \mathrm{~J}_{\mathrm{H}, \mathrm{H}}=7.0 \mathrm{~Hz}, 4 \mathrm{H}, \mathrm{OCH}_{2}\right), 3.28$ - $3.08\left(\mathrm{~m}, 4 \mathrm{H}, \mathrm{PCH}_{2}\right), 1.15\left(\mathrm{t},{ }^{3} \mathrm{~J}_{\mathrm{H}, \mathrm{H}}=7.0 \mathrm{~Hz}, 6 \mathrm{H}, \mathrm{CH}_{3} \mathrm{CH}_{2} \mathrm{O}\right) ;{ }^{13} \mathrm{C}\left\{{ }^{1} \mathrm{H}\right\} \mathrm{NMR}\left(151 \mathrm{MHz}, \mathrm{CD}_{2} \mathrm{Cl}_{2}\right): \delta[p p m]=151.4$ (s, $\operatorname{ArC}), 136.6(s, \operatorname{ArC}), 133.9\left(d, J_{P, C}=2.7 \mathrm{~Hz}, \operatorname{ArC}\right), 132.6\left(d, J_{P, C}=10.7 \mathrm{~Hz}, \operatorname{ArC}\right), 131.9\left(d, J_{P, C}=2.1 \mathrm{~Hz}, \operatorname{ArC}\right)$, $131.8\left(d, J_{P, C}=5.3 \mathrm{~Hz}, \operatorname{ArC}\right), 130.8\left(\mathrm{~d}, J_{\mathrm{P}, \mathrm{C}}=2.8 \mathrm{~Hz}, \operatorname{ArC}\right), 129.7$ (d, $J_{\mathrm{P}, \mathrm{C}}=5.3 \mathrm{~Hz}, \operatorname{ArC}$ ), 129.6 (s, ArC), 129.0 (d, $\left.J_{P, C}=1.5 \mathrm{~Hz}, \mathrm{ArC}\right), 128.9(\mathrm{~s}, m-\mathrm{NPh}), 126-0(\mathrm{~s}, \operatorname{ArC}), 121.6(\mathrm{~s}, p-\mathrm{NPh}), 117.8(\mathrm{~s}, m-\mathrm{NPh}), 30.2\left(\mathrm{~d},{ }^{1} J_{\mathrm{P}, \mathrm{C}}=11.9\right.$ $\mathrm{Hz}, \mathrm{PCH}_{2}$ ), $21.9\left(\mathrm{~s}, \mathrm{OCH}_{2}\right), 15.7\left(\mathrm{~s}, \mathrm{OCH}_{2} \mathrm{CH}_{3}\right) ;{ }^{31} \mathrm{P}\left\{{ }^{1} \mathrm{H}\right\} \mathrm{NMR}\left(243 \mathrm{MHz}, \mathrm{CD}_{2} \mathrm{Cl}_{2}\right.$ ): $\delta$ [ppm] = 14.4 (s); ); Elemental analysis calcd. for $\mathrm{C}_{36} \mathrm{H}_{37} \mathrm{Cl}_{2} \mathrm{HfN}_{2} \mathrm{OP}$ : $\mathrm{C} 54.45, \mathrm{H} 4.70, \mathrm{~N} 3.53$; found $\mathrm{C} 54.44, \mathrm{H} 4.43, \mathrm{~N} 3.58$. 


\section{2) Additional ORTEP Diagrams}

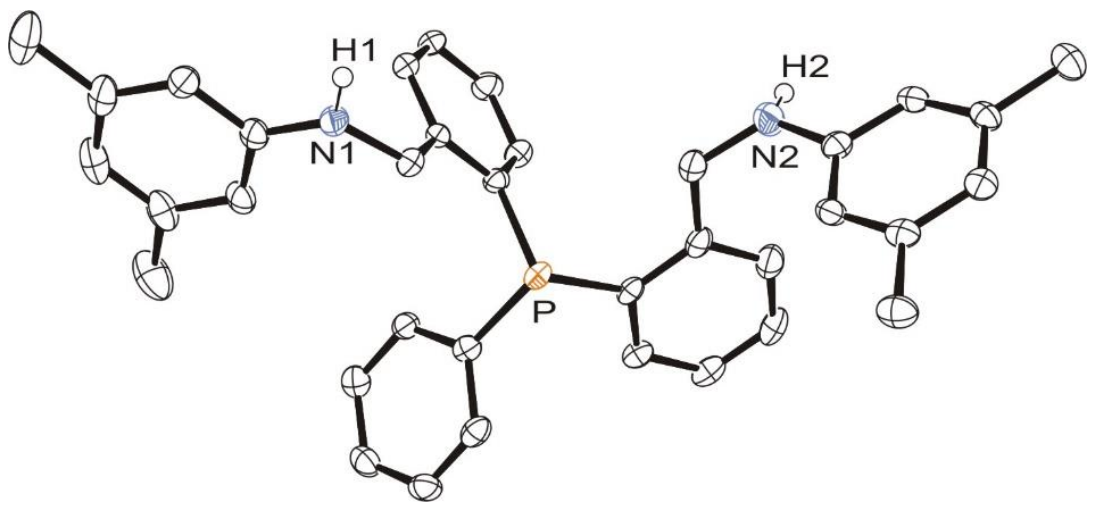

Fig. S1. ORTEP diagram of $[\mathrm{B}] \mathrm{H}_{2}$ (hydrogen atoms except $\mathrm{H} 1$ and $\mathrm{H} 2$ have been omitted for clarity, displacement ellipsoids set at $50 \%$ probability). Values in square brackets refer to the second independent molecule. Selected bond lengths $(\AA)$ and angles $\left({ }^{\circ}\right)$ : P-C 1.836(2), 1.837(2) and 1.831(2) [1.836(2), 1.838(2) and 1.830(2)], N1-H1 0.81(2) [0.80(2)], N2-H2 0.87(3) [0.87(3)], C-P-C 104.19(10), 100.43(10) and 103.75(10) [104.71(10), 102.83(11) and 100.91(10)].
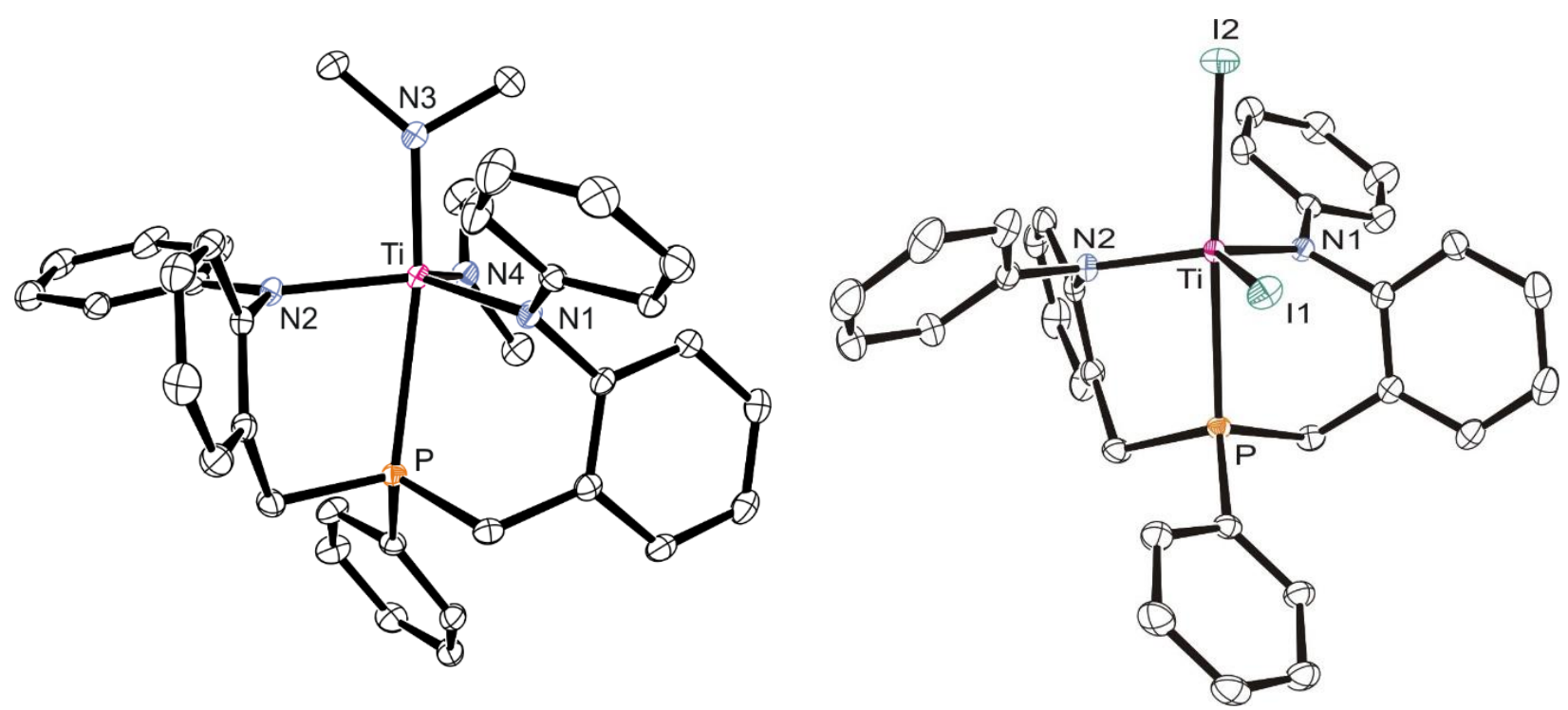

Fig. S2. ORTEP diagrams of $\left[{ }^{\mathrm{Ph}} \mathrm{A}\right]-\mathbf{- 1}-\mathrm{Ti}$ and $\left[{ }^{\mathrm{Ph}} \mathrm{A}\right]-2-\mathrm{Ti}$ (hydrogen atoms omitted for clarity, displacement ellipsoids set at $50 \%$ probability). Selected bond lengths $(\AA)$ and angles $\left({ }^{\circ}\right)$ of $\left[{ }^{\mathrm{Ph}} \mathrm{A}\right]-1-\mathrm{Ti}$ : Ti-P $2.7143(10)$, Ti-N2 2.0067(13), Ti-N1 2.0254(13), Ti-N3 1.9121(14), Ti-N4 1.9179(15), N2-Ti-N1 127.77(5), N3-Ti-P 166.67(4), N4-Ti-P 95.51(4). Selected bond lengths ( $(\AA)$ and angles $\left({ }^{\circ}\right)$ of [ $\left.{ }^{\mathrm{Ph}} \mathrm{A}\right]-2-\mathrm{Ti}:$ Ti-P 2.6182(5), Ti-N1 1.9371(13), Ti-N2 1.9244(13), Ti-I1 2.6841(3), Ti-I2 2.7130(3), I1-Ti-I2 94.420(9), P-Ti-I1 87.840(12), P-Ti-I2 175.591(14), N1-Ti-N2 118.37(6). 

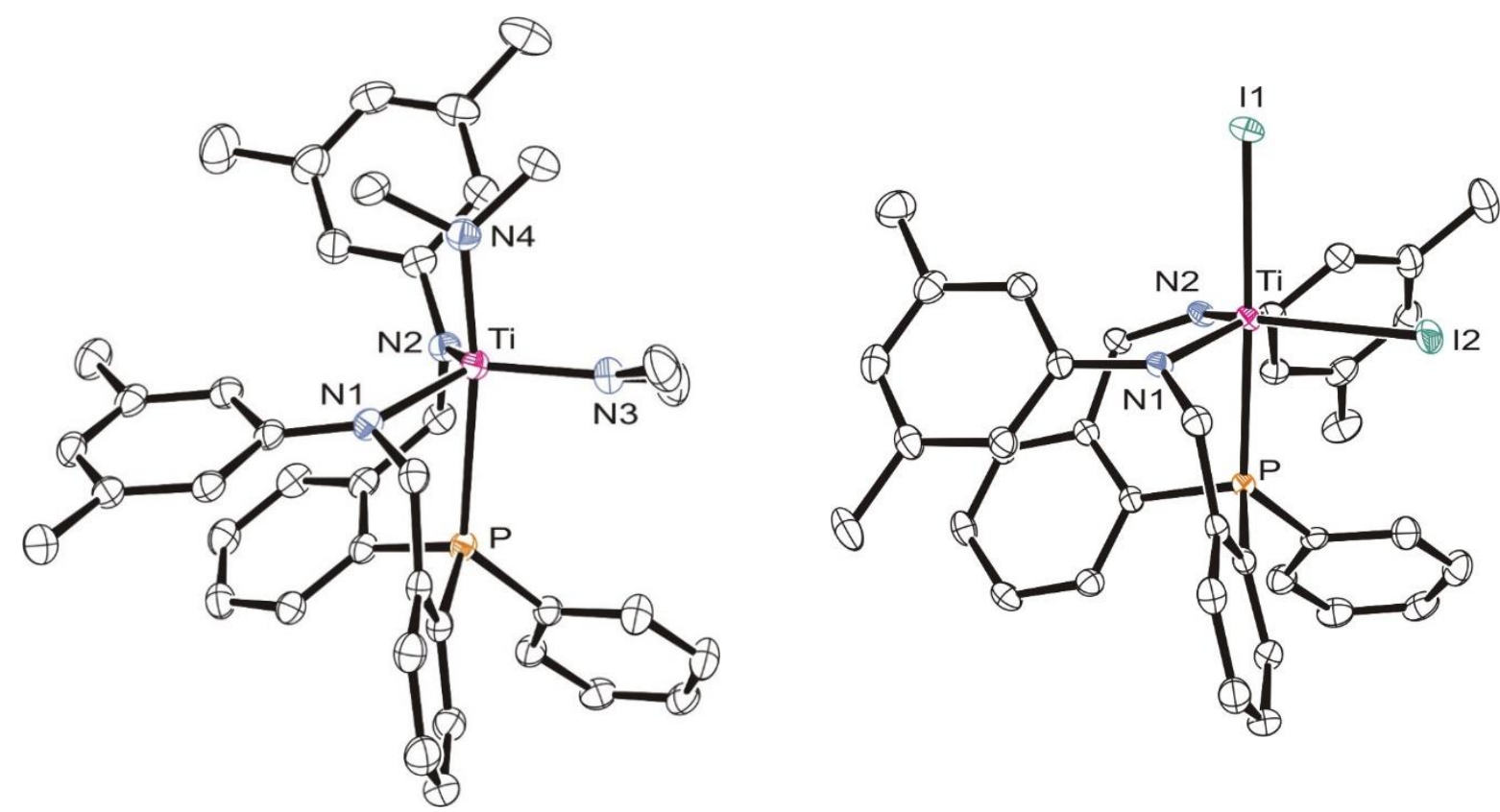

Fig. S3. ORTEP diagrams of [B]-1-Ti and [B]-2-Ti (hydrogen atoms omitted for clarity, displacement ellipsoids set at $50 \%$ probability). Selected bond lengths ( $(\AA)$ and angles $\left({ }^{\circ}\right)$ of [B]-1-Ti: Ti-P 2.6987(4), Ti-N1 2.0245(12), Ti-N2 1.9554(11), Ti-N3 1.9262(12), Ti-N4 1.9311(12), N2-Ti-N1 114.60(5), N3-Ti-P 91.28(4), N4-Ti-P 170.16(4). Selected bond lengths ( $\AA$ ) and angles ( $\left(^{\circ}\right)$ of [B]-2-Ti: Ti-P 2.5365(6), Ti-N1 1.9050(16), Ti-N2 1.9079(16), Ti-I1 2.7828(3), Ti-I2 2.7442(4), I1-Ti-I2 90.966(10), P-Ti-I1 177.480(18), P-Ti-I2 90.302(15), N1-Ti-N2 112.80(7).
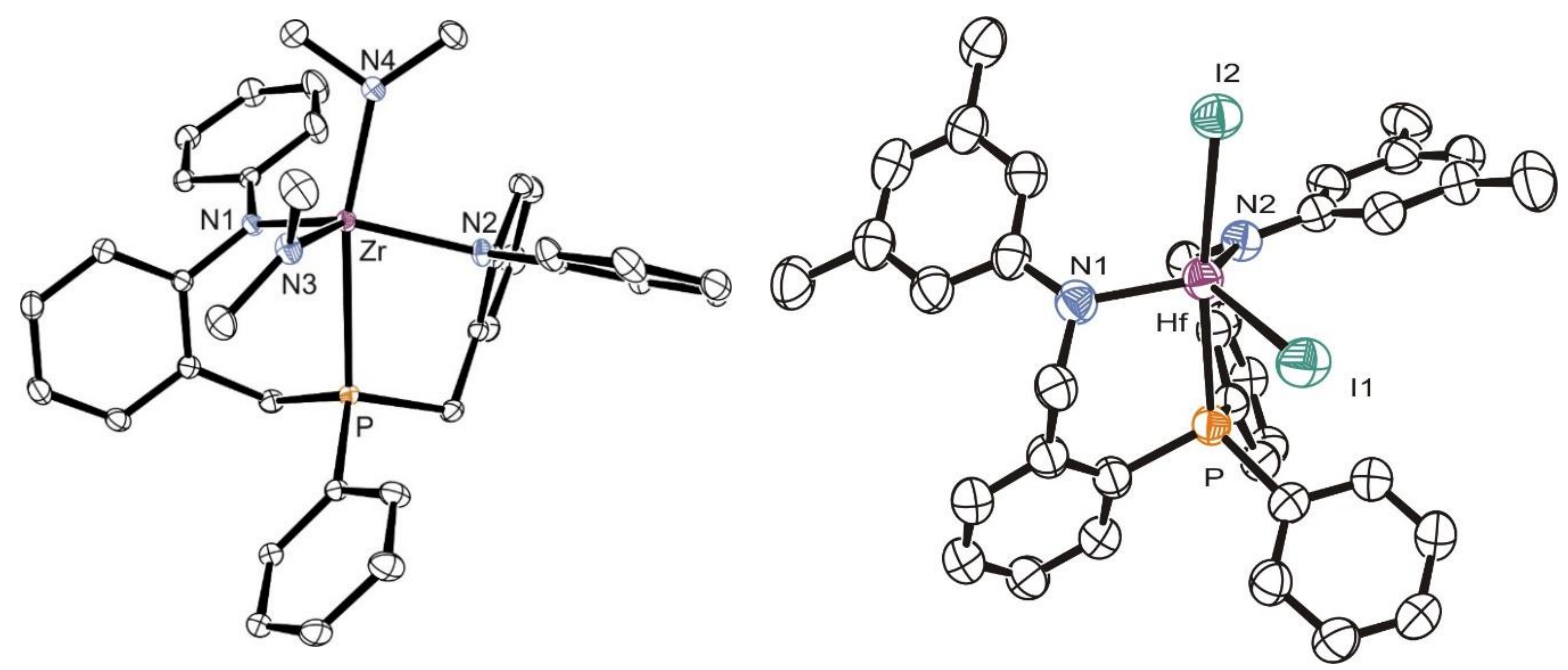

Fig. S4. ORTEP diagrams of $\left[{ }^{\mathrm{Ph}} \mathrm{A}\right]-\mathbf{1}-\mathrm{Zr}$ and [B]-2-Hf (hydrogen atoms omitted for clarity, displacement ellipsoids set at $50 \%$ probability). Selected bond lengths $(\AA)$ and angles $\left({ }^{\circ}\right)$ of $\left[{ }^{\mathrm{Ph}} \mathrm{A}\right]-1-\mathrm{Zr}$ : Zr-P 2.8305(9), Zr-N1 2.1601(11), Zr-N2 2.1442(10), Zr-N3 2.0488(13), Zr-N4 2.0430(11), N2-Zr-N1 125.31(4), N3-Zr-P 95.03(3), N4-Zr-P 163.85(3). Selected bond lengths ( $\AA$ ) and angles $\left({ }^{\circ}\right)$ of [B]-2-Hf: Hf-P 2.7448(11), Hf-N1 2.033(4), Hf-N2 2.027(3), Hf-I1 2.7859(4), Hf-I2 2.8080(3), I1-Hf-I2 91.198(11), P-Hf-I1 85.88(2), P-Hf-I2 172.64(2), N2-Hf-N1 110.38(15). 

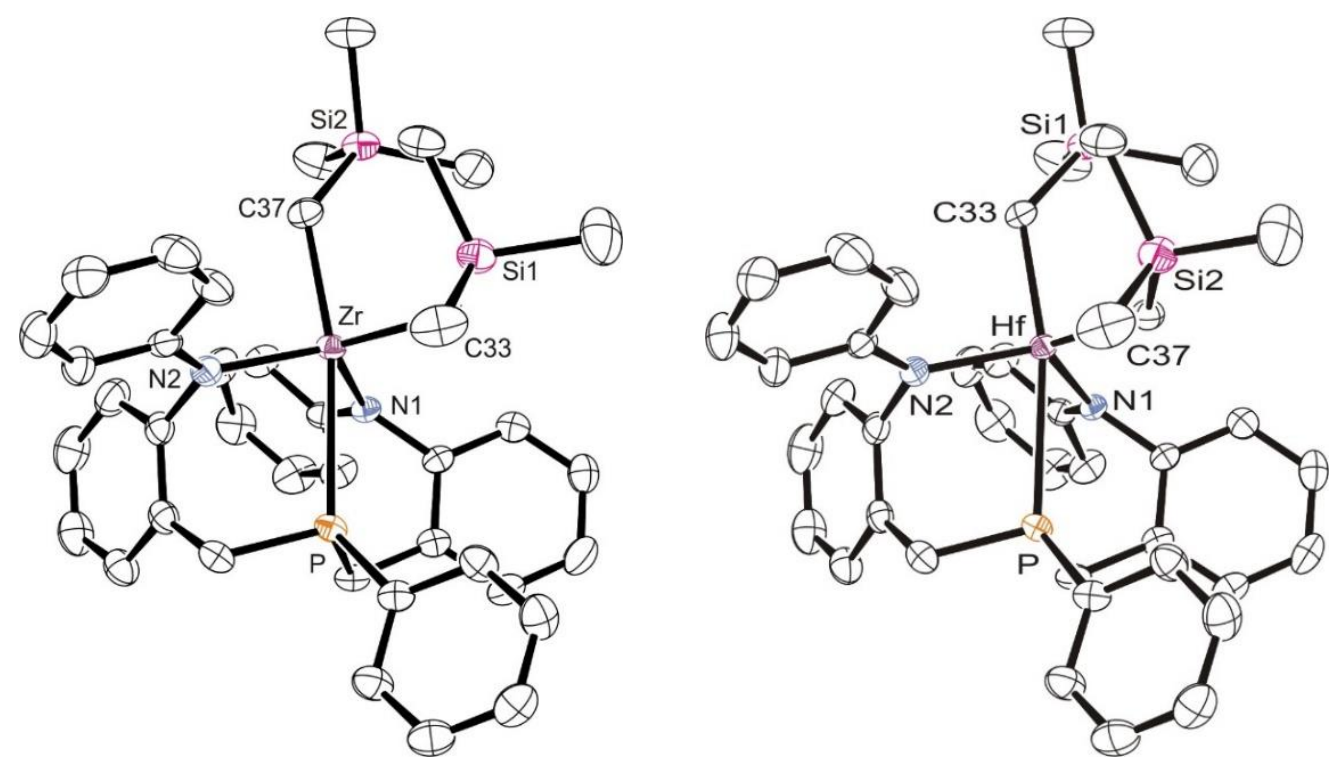

Fig. S5. ORTEP diagrams of $\left[{ }^{\mathrm{Ph}} \mathbf{A}\right]-\mathbf{3}-\mathrm{Zr}$ and $\left[{ }^{\mathrm{Ph}} \mathbf{A}\right]-\mathbf{3}-\mathrm{Hf}$ (hydrogen atoms omitted for clarity, displacement ellipsoids set at $50 \%$ probability). Selected bond lengths $(\AA \AA)$ and angles $\left({ }^{\circ}\right)$ of $\left[{ }^{\mathrm{Ph}} \mathrm{A}\right]-3-\mathrm{Zr}$ : $\mathrm{Zr}-\mathrm{P} 2.9485(6)$, $\mathrm{Zr}-\mathrm{N} 1$ 2.088(2), Zr-N2 2.109(2), Zr-C33 2.229(2), Zr-C37 2.254(2), N1-Zr-N2 117.08(8), C33-Zr-P 92.36(6), C37-Zr-P 169.08(7), C33-Zr-C37 94.55(9). Selected bond lengths (Å) and angles $\left({ }^{\circ}\right)$ of [ $\left.{ }^{\mathrm{Ph}} \mathrm{A}\right]-3-\mathrm{Hf}$ : $\mathrm{Hf}-\mathrm{P}$ 2.9379(7), Hf-N1 2.085(2), Hf-N2 2.105(2), Hf-C33 2.239(3), Hf-C37 2.208(3), N1-Hf-N2 117.95(9), C33-Hf-P 169.20(8), C37-Hf-P 92.47(8), C37-Hf-C33 94.87(11).
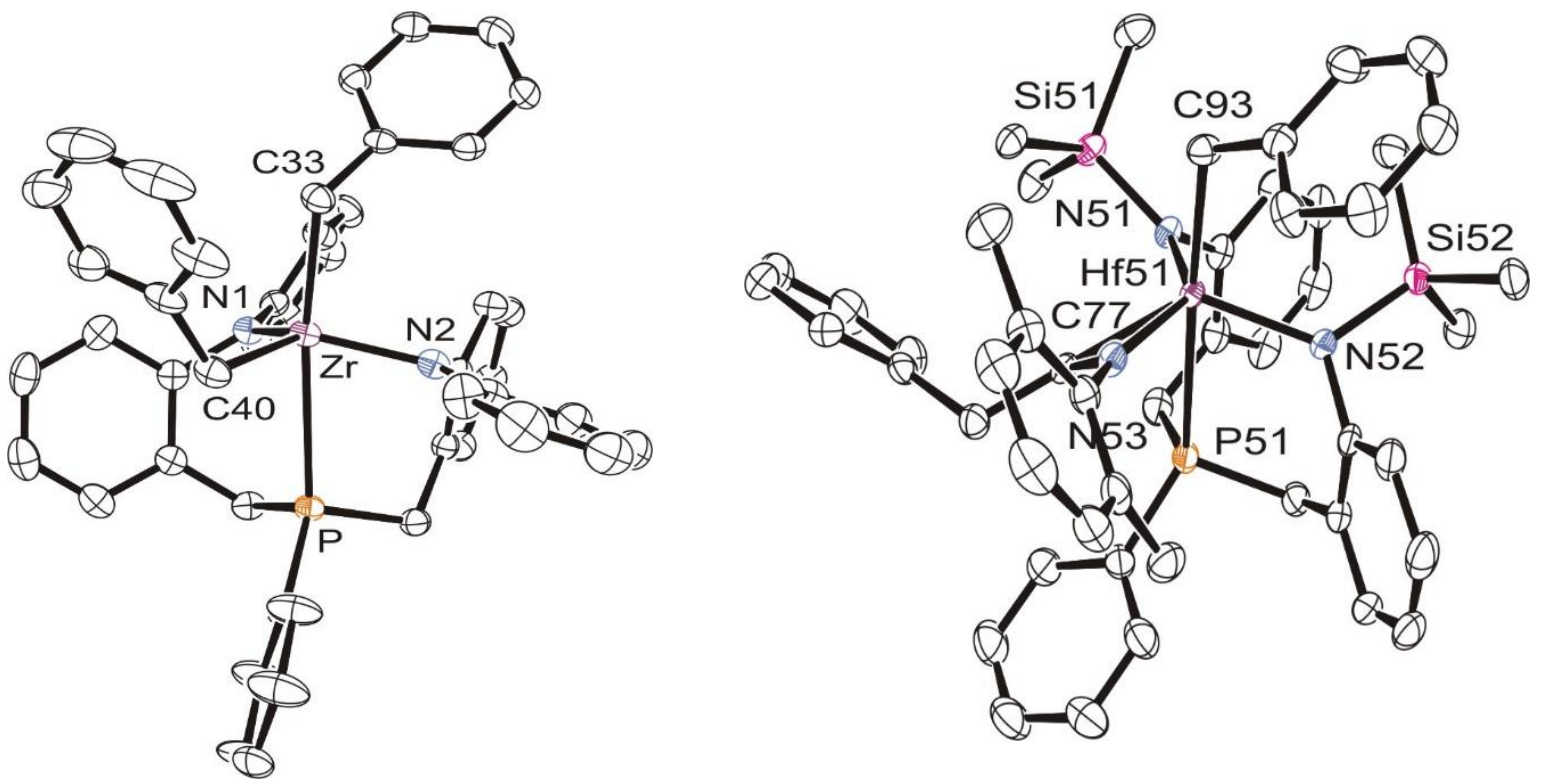

Fig. S6. ORTEP diagrams of $\left[{ }^{\mathrm{Ph}} \mathrm{A}\right]-4-\mathrm{Zr}$ and $\left[{ }^{\mathrm{Si}} \mathrm{A}\right]-6-\mathrm{Hf}$ (second molecule in the unit cell, c.f. Table 4 in the article) (hydrogen atoms and disordered PPh carbons omitted for clarity, displacement ellipsoids set at 50 $\%$ probability). Selected bond lengths ( $(\AA)$ and angles $\left({ }^{\circ}\right)$ of [ $\left.{ }^{\mathrm{Ph}} \mathrm{A}\right]-4-\mathrm{Zr}$ : Zr-P 2.8708(5), Zr-N1 2.0785(14), Zr-N2 2.0870(16), Zr-C33 2.2985(19), Zr-C40 2.2892(18), N1-Zr-N2 118.68(6), C33-Zr-P 168.05(5), C40-Zr-P 85.93(5), C33-Zr-C37 89.98(7). Selected bond lengths $(\AA \AA)$ and angles $\left({ }^{\circ}\right)$ of $\left[{ }^{\mathrm{Si}} \mathrm{A}\right]-6-\mathrm{Hf}$ (second molecule in the unit cell, c.f. Table 4 in the article): Hf51-P51 2.8568(7), Hf51-N51 2.097(2), Hf51-N52 2.081(2), Hf51-N53 2.252(2) Hf51-C77 2.247(3), N53-Hf51-P51 94.88(6), Hf51-C93 2.315(3), N52-Hf51-N51 109.16(8), C77-Hf51-P51 76.13(7), C93-Hf51-P51 177.42(7). 

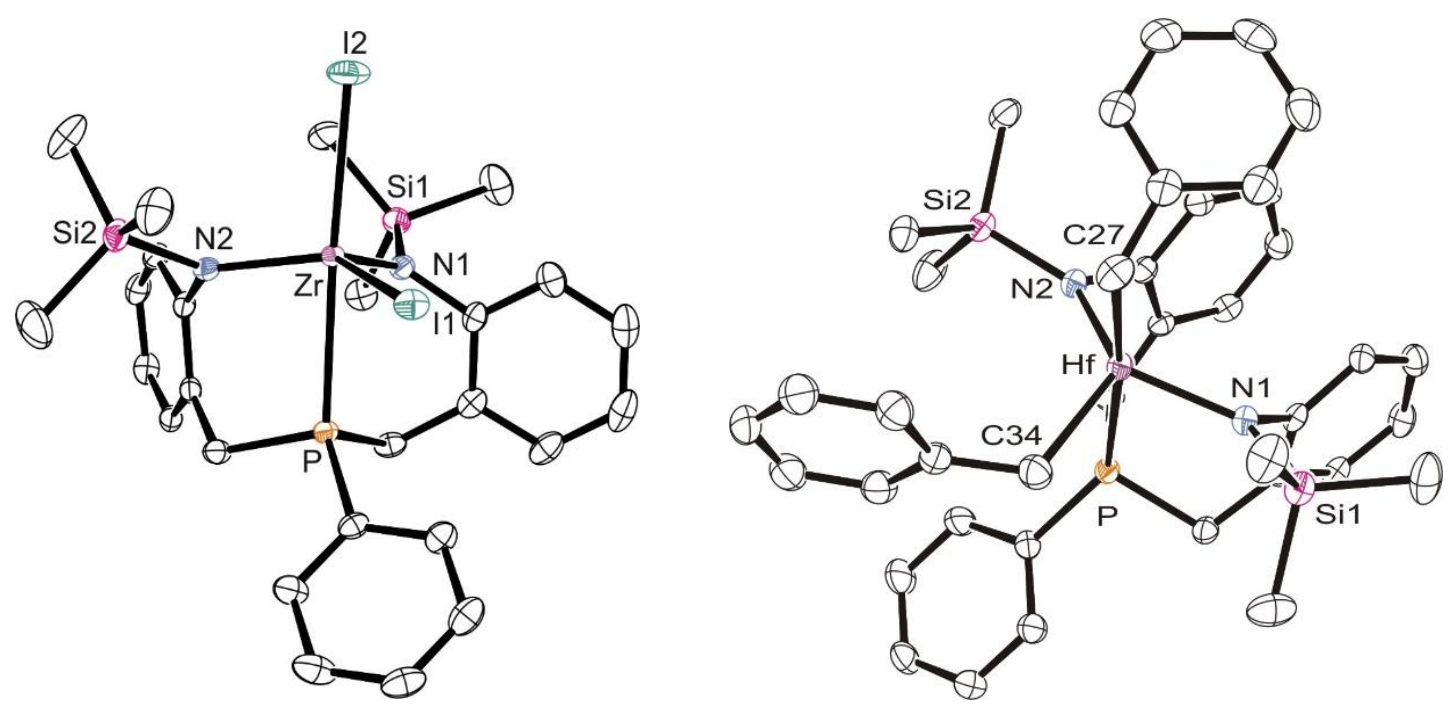

Fig. S7. ORTEP diagrams of $\left[{ }^{\mathrm{Si}} \mathrm{A}\right]-\mathbf{2}-\mathrm{Zr}$ and $\left[{ }^{\mathrm{Si}} \mathrm{A}\right]-\mathbf{4}-\mathrm{Hf}\left(C_{S}\right)$ (hydrogen atoms omitted for clarity, displacement

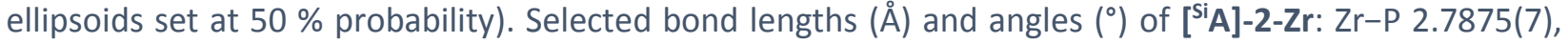
Zr-N1 2.019(2), Zr-N2 2.051(2), Zr-I1 2.8356(3), Zr-I2 2.8595(3), N1-Zr-N2 109.68(9), I1-Zr-P 82.774(15), I2-Zr-P 175.861(17), I2-Zr-I1 93.203(9). Selected bond lengths $(\AA ̊)$ and angles $\left({ }^{\circ}\right)$ of $\left[{ }^{\text {Si }} A\right]-4-H f\left(C_{S}\right): H f-P$ 2.8232(6), Hf-N1 2.073(2), Hf-N2 2.068(2), Hf-C27 2.278(3), Hf-C34 2.264(3), N2-Hf-N1 124.21(8), C27-Hf-P 174.37(8), C34-Hf-P 86.27(8), C34-Hf-C27 90.90(11).
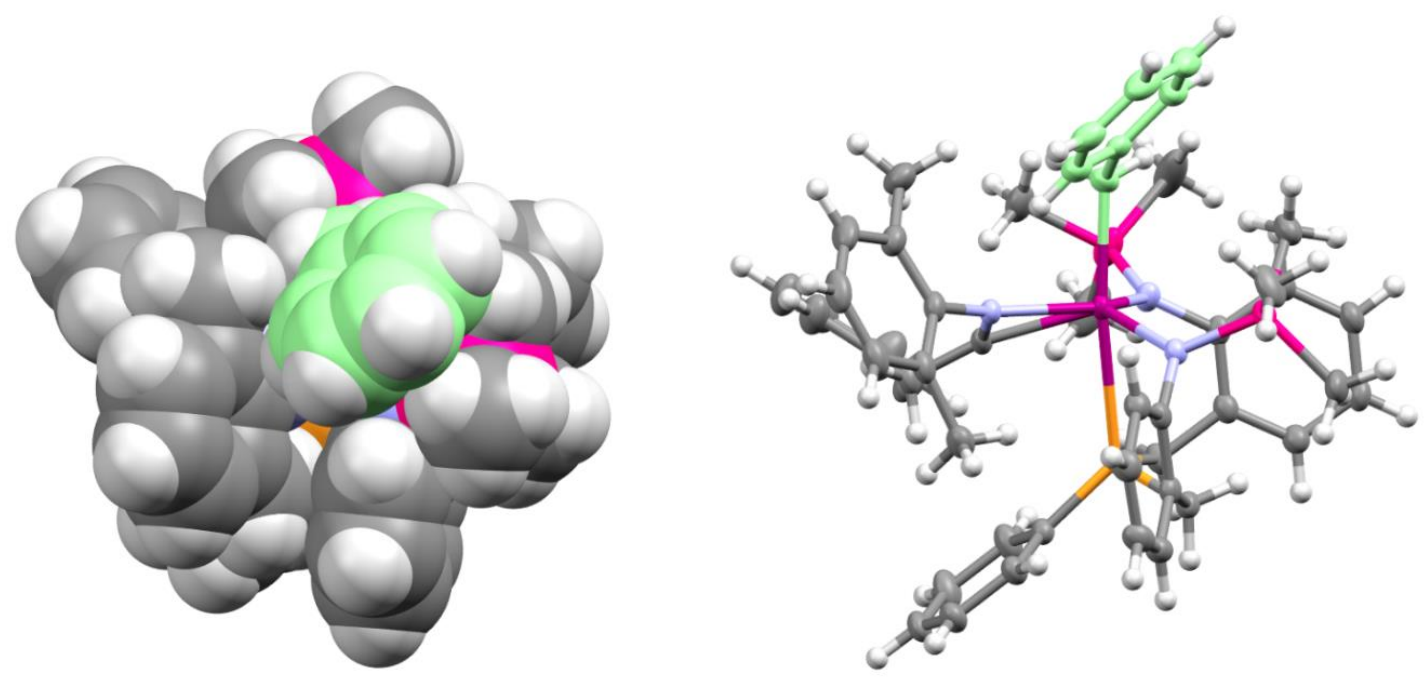

Fig. S8. Space-filling representation of $\left[{ }^{\text {Si } A]-6-H f}\right.$ (top) together with the corresponding ellipsoid representation (bottom) (displacement ellipsoids set at $50 \%$ probability). The trans-P-positioned benzylic $\mathrm{Hf}-\mathrm{CH}_{2}$ group (highlighted in light green) is sterically shielded by the two $\mathrm{N}$-trimethylsilyl substituents of the ligand, which might explain its lack of reactivity in isonitrile insertion reactions.

\section{3) Selected NMR Spectra}

Selected ${ }^{1} \mathrm{H}$ NMR spectra and ${ }^{31} \mathrm{P}\left\{{ }^{1} \mathrm{H}\right\}$ NMR spectra of the protioligands $\left[{ }^{\mathrm{Ph}} \mathrm{A}\right] \mathrm{H}_{2}$ and $[\mathbf{B}] \mathrm{H}_{2}$ and of the complexes [ $\left.{ }^{\mathrm{Ph}} \mathrm{A}\right]-\mathbf{- 1}-\mathrm{M},[\mathrm{B}]-\mathbf{- 1}-\mathrm{M},\left[{ }^{\mathrm{Ph}} \mathrm{A}\right]-\mathbf{- 2}-\mathrm{M},[\mathrm{B}]-\mathbf{2}-\mathrm{M},\left[{ }^{\mathrm{Ph}} \mathrm{A}\right]-\mathbf{3}-\mathrm{M},\left[{ }^{\mathrm{Ph}} \mathrm{A}\right]-\mathbf{4}-\mathrm{M},[\mathrm{B}]-\mathbf{4}-\mathrm{M},\left[{ }^{\mathrm{Si}} \mathrm{A}\right]-\mathbf{4}-\mathrm{Hf},[\mathrm{B}]-\mathbf{5}-\mathrm{Zr}$, $\left[{ }^{\mathrm{Ph}} \mathrm{A}\right]-\mathbf{6}-\mathbf{M},[\mathrm{B}]-\mathbf{6}-\mathbf{H f}$ and $\left[{ }^{\mathrm{Si}} \mathrm{A}\right]-\mathbf{6}-\mathbf{H f}$ (one example for in case of isostructural complexes ) are provided on the following pages. 
<smiles>CC(C)P(c1ccccc1)c1ccccc1Nc1ccccc1</smiles>

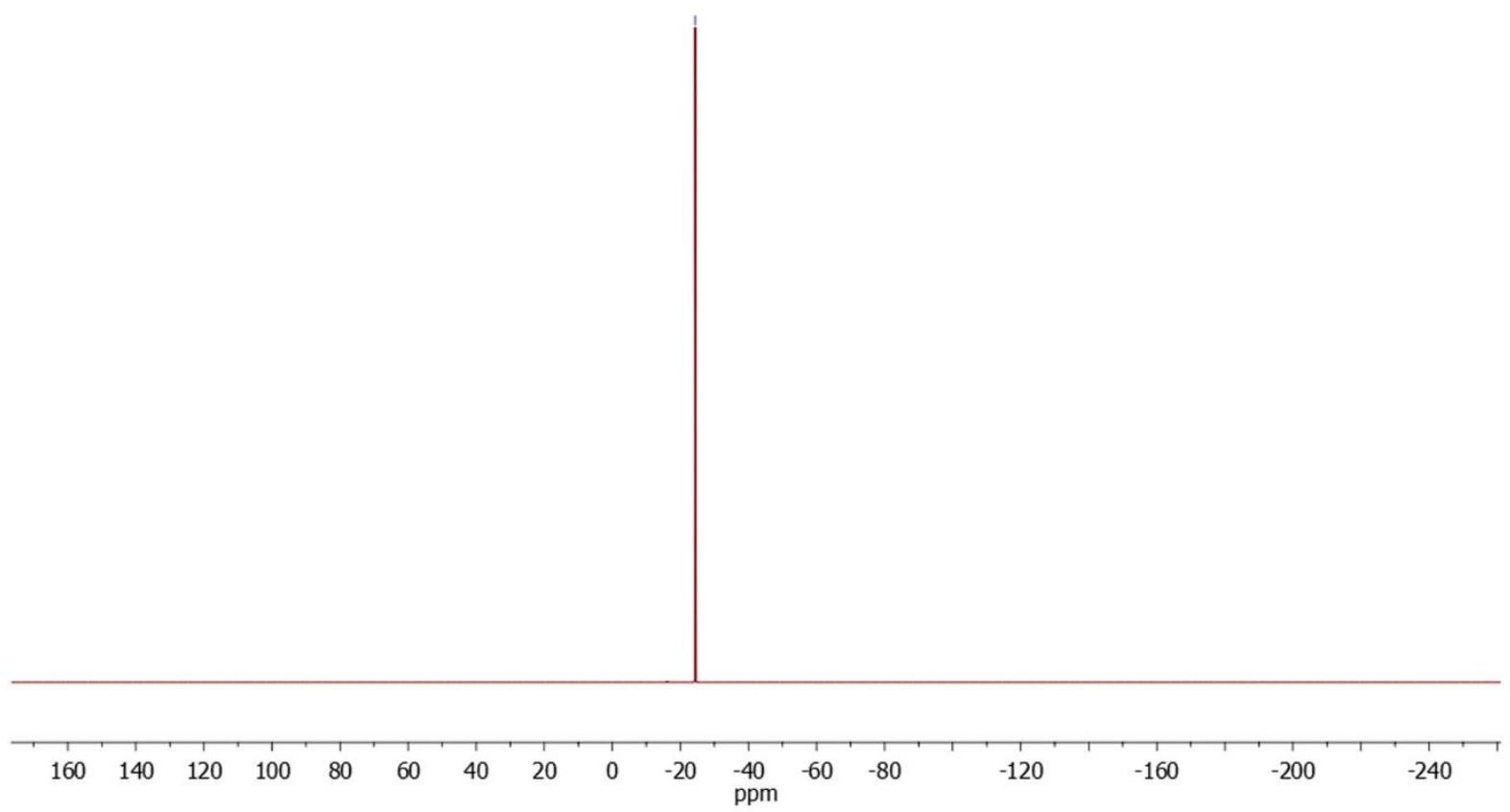

Fig. S9. ${ }^{31} \mathrm{P}\left\{{ }^{1} \mathrm{H}\right\} \mathrm{NMR}\left(243 \mathrm{MHz}, \mathrm{C}_{6} \mathrm{D}_{6}\right)$ of $\left[{ }^{\mathrm{Ph}} \mathrm{A}\right] \mathrm{H}_{2}$.

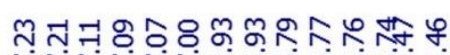

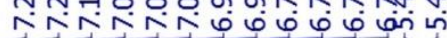<smiles>CC(c1ccccc1)c1ccccc1Nc1ccccc1</smiles>
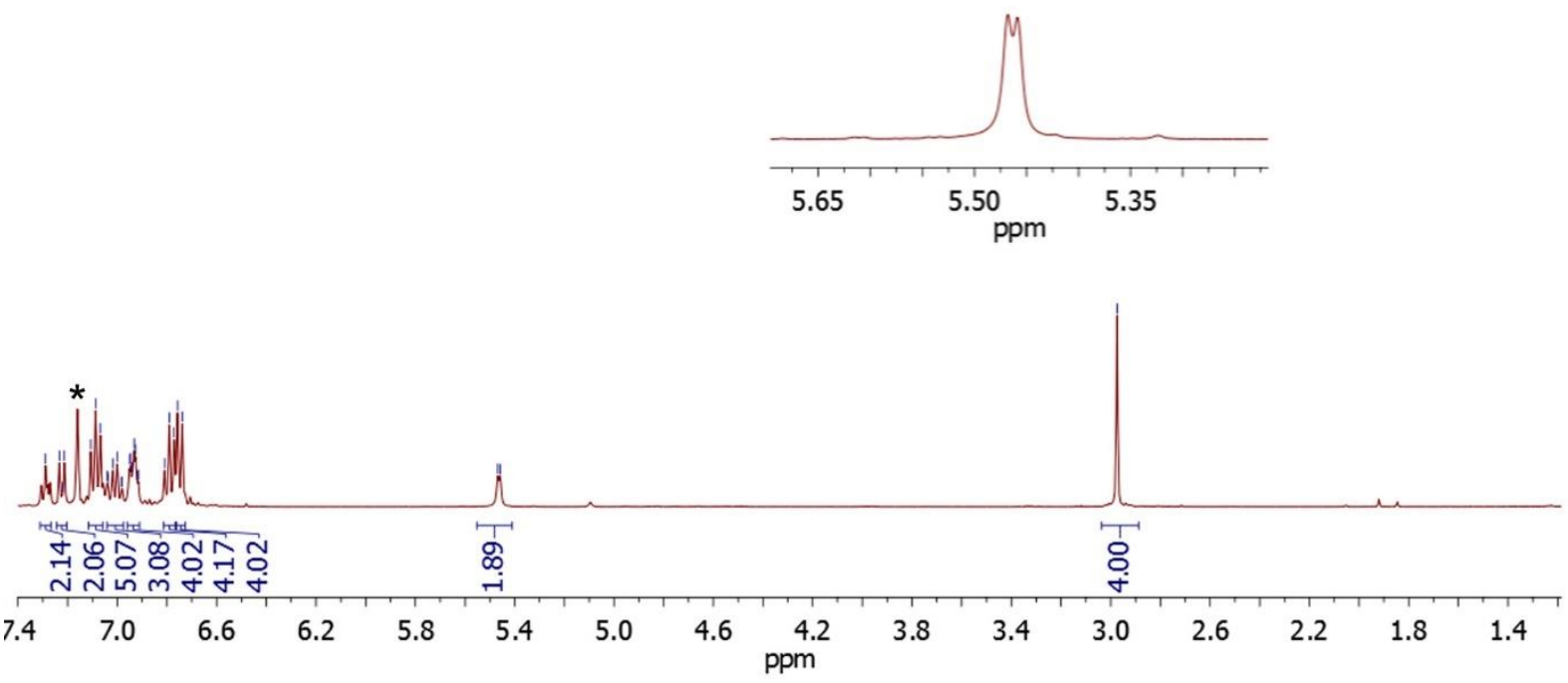

Fig. S10. ${ }^{1} \mathrm{H}$ NMR (600 MHz, $\mathrm{C}_{6} \mathrm{D}_{6}$ ) of $\left[{ }^{\mathrm{Ph}} \mathrm{A}\right] \mathrm{H}_{2}$ (residual proton signal of $\mathrm{C}_{6} \mathrm{D}_{6}$ is labeled with $*$ ). 

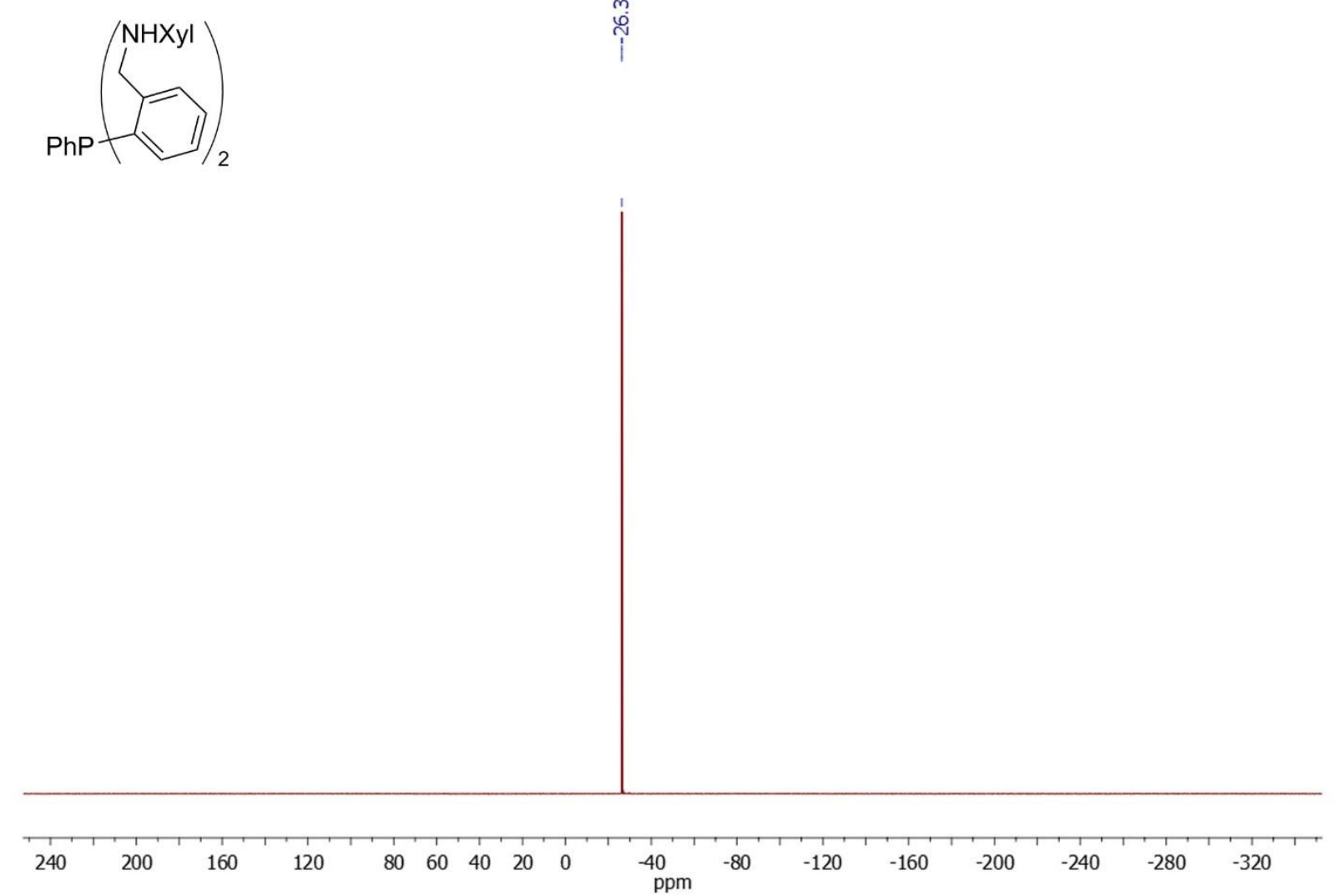

Fig. S11. ${ }^{31} \mathrm{P}\left\{{ }^{1} \mathrm{H}\right\} \mathrm{NMR}\left(243 \mathrm{MHz}, \mathrm{C}_{6} \mathrm{D}_{6}\right)$ of $[\mathrm{B}] \mathrm{H}_{2}$.

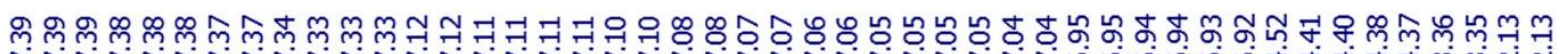

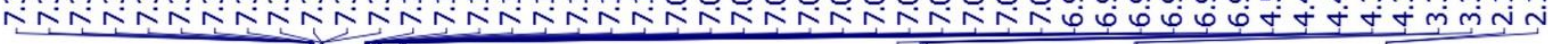<smiles>Cc1ccccc1CNCCc1ccccc1</smiles>
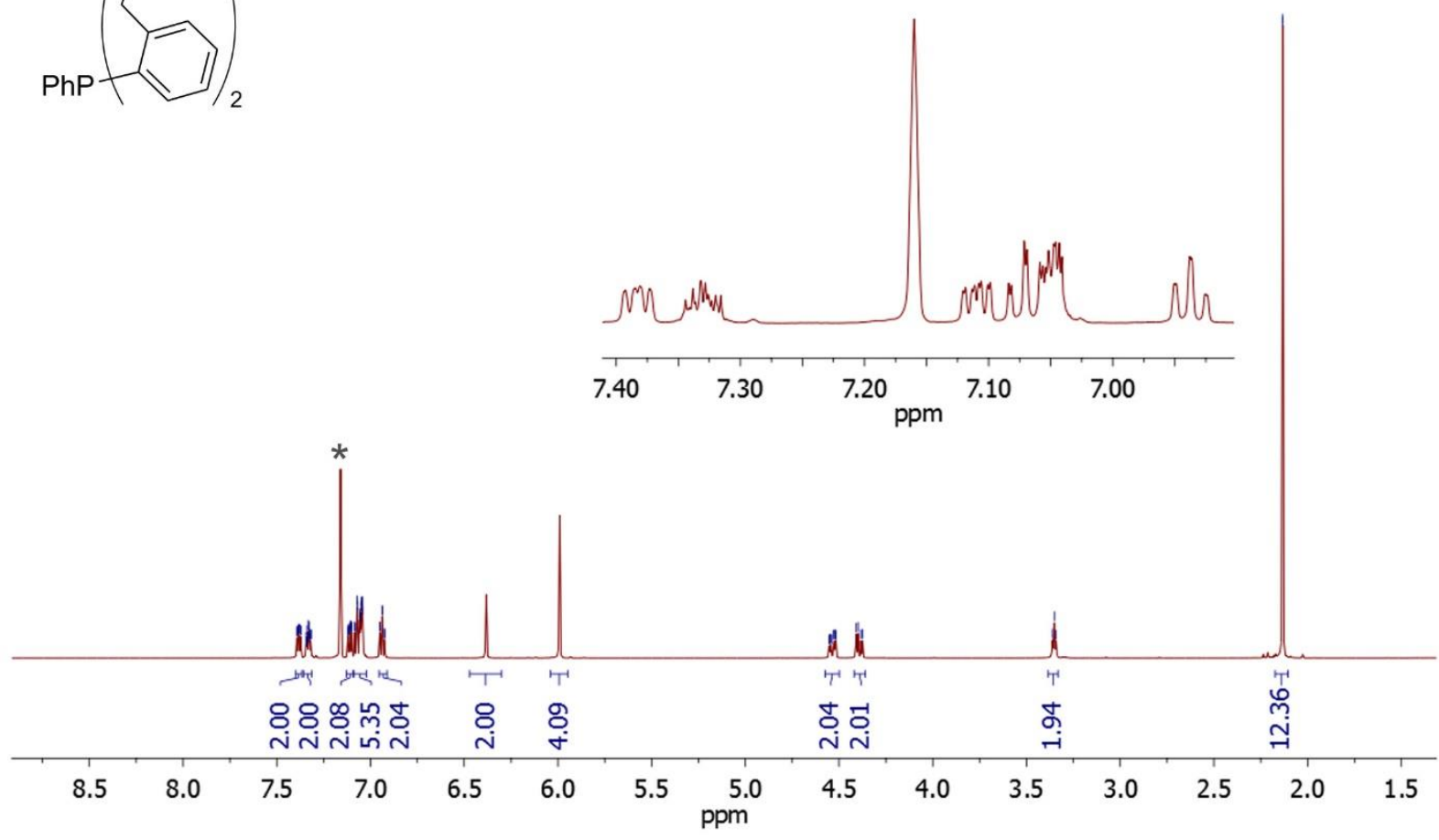

Fig. S12. ${ }^{1} \mathrm{H} N M R\left(600 \mathrm{MHz}, \mathrm{C}_{6} \mathrm{D}_{6}\right)$ of $[\mathrm{B}] \mathrm{H}_{2}$ (residual proton signal of $\mathrm{C}_{6} \mathrm{D}_{6}$ is labeled with $*$ ). 

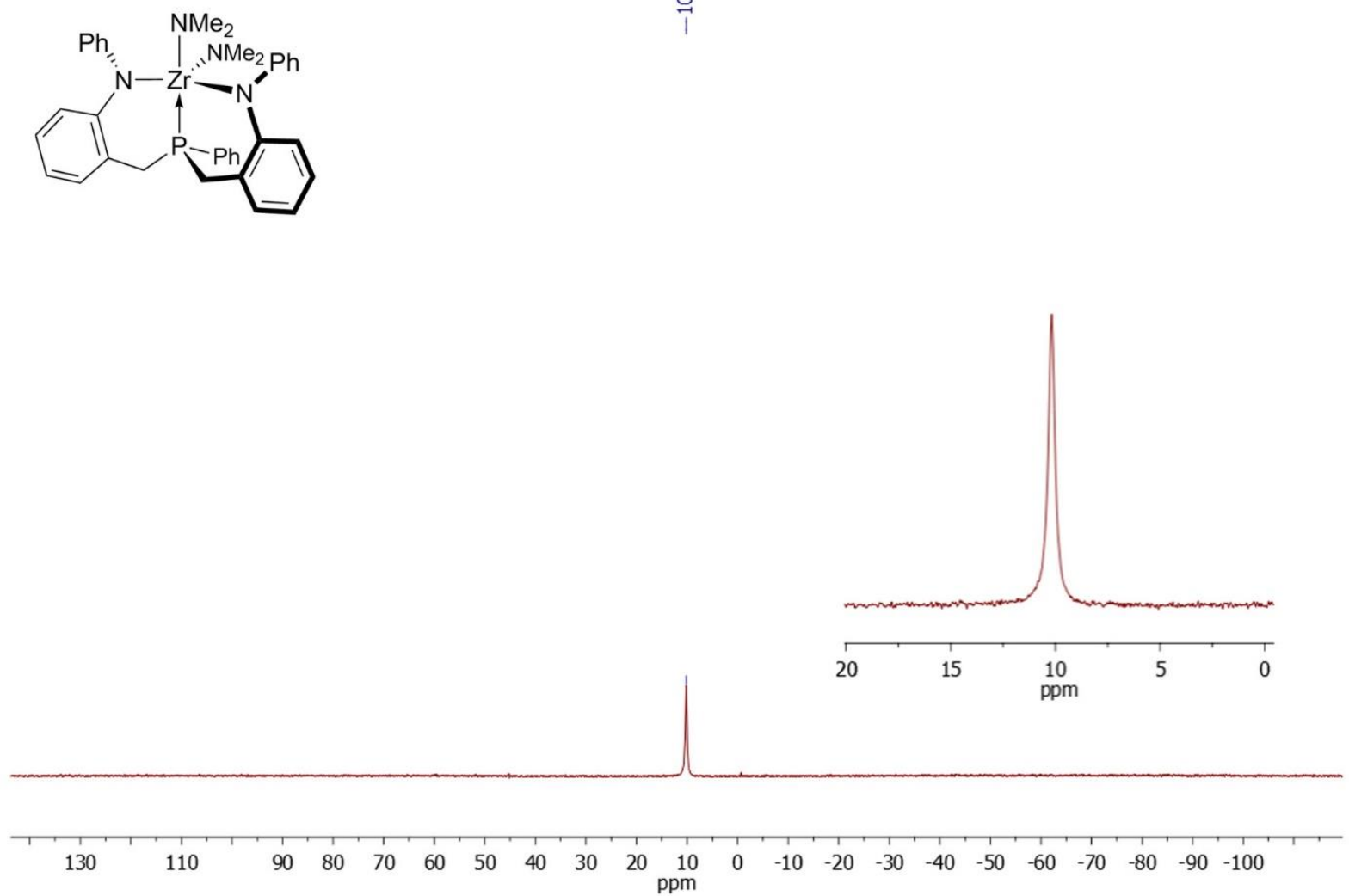

Fig. S13. ${ }^{31} \mathrm{P}\left\{{ }^{1} \mathrm{H}\right\} \mathrm{NMR}\left(243 \mathrm{MHz}, \mathrm{C}_{6} \mathrm{D}_{6}\right)$ of $\left[{ }^{\mathrm{Ph}} \mathrm{A}\right]-1-\mathrm{Zr}$.

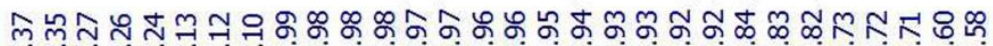

NNNNNNNN

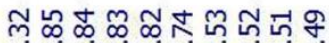
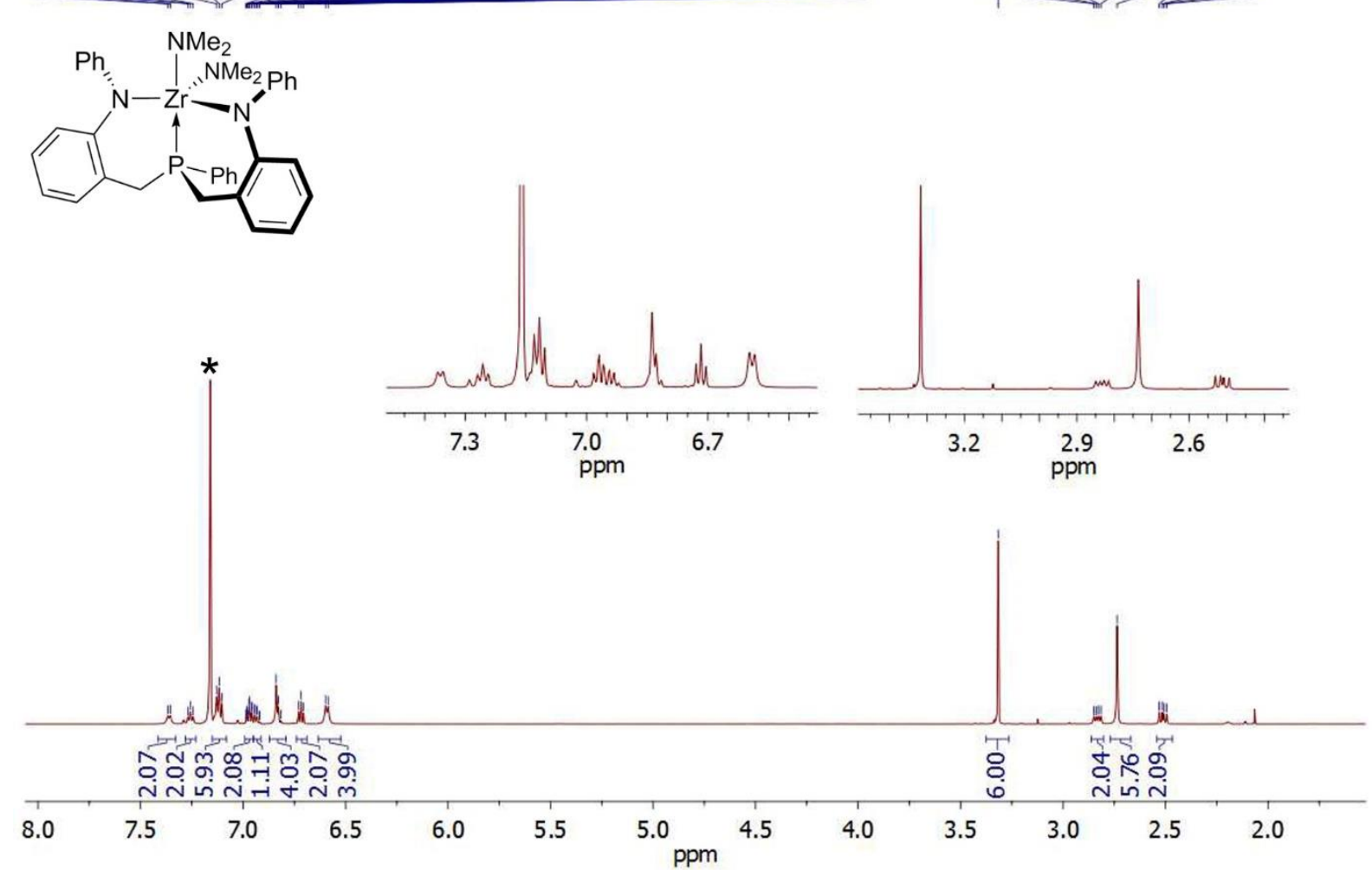

miñ

Fig. S14. ${ }^{1} \mathrm{H}$ NMR $\left(600 \mathrm{MHz}, \mathrm{C}_{6} \mathrm{D}_{6}\right)$ of $\left[{ }^{\mathrm{Ph}} \mathrm{A}\right]-1-\mathrm{Zr}$ (residual proton signal of $\mathrm{C}_{6} \mathrm{D}_{6}$ is labeled with $*$ ). 

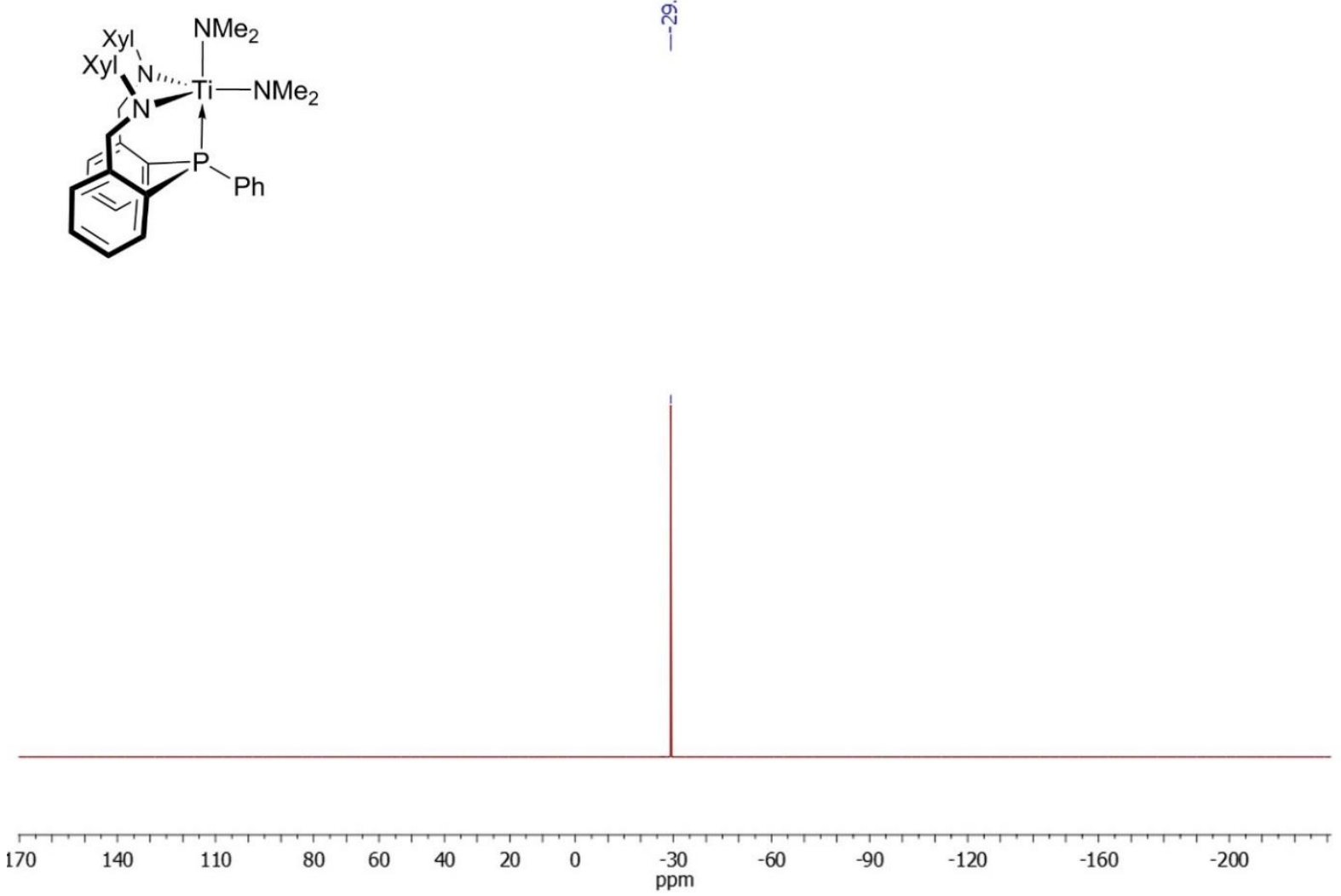

Fig. S15. ${ }^{31} \mathrm{P}\left\{{ }^{1} \mathrm{H}\right\}$ NMR (162 MHz, $\left.\mathrm{C}_{6} \mathrm{D}_{6}\right)$ of [B]-1-Ti.

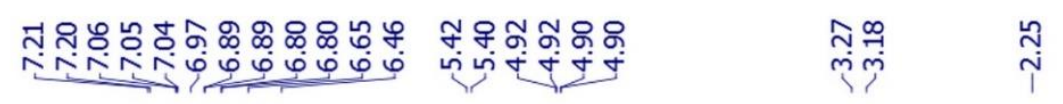

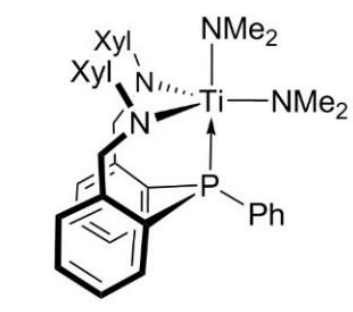

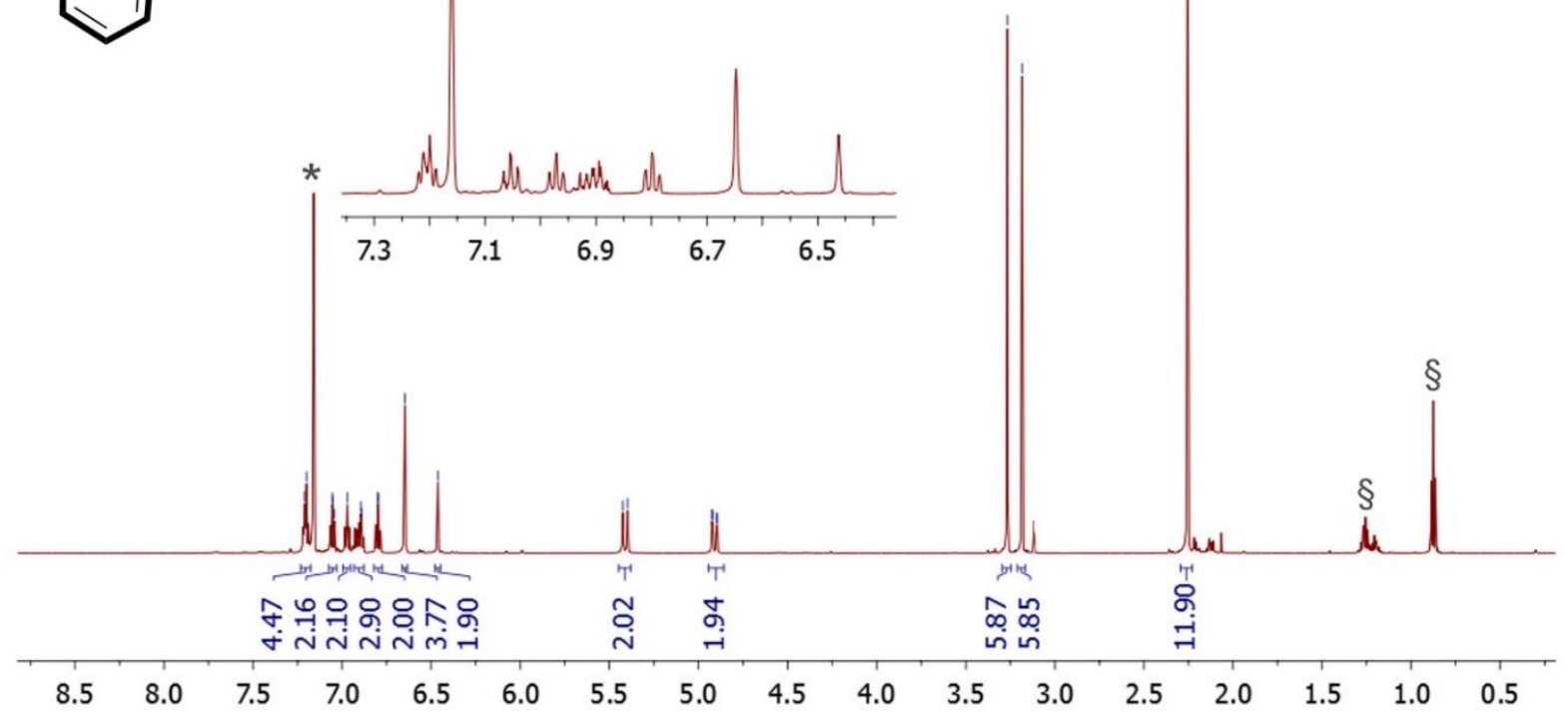

Fig. S16. ${ }^{1} \mathrm{H}$ NMR $\left(600 \mathrm{MHz}, \mathrm{C}_{6} \mathrm{D}_{6}\right)$ of $[\mathrm{B}]-1-\mathrm{Ti}$ (residual proton signal of $\mathrm{C}_{6} \mathrm{D}_{6}$ is labeled with $*$, residual pentane signals are labeled with §). 

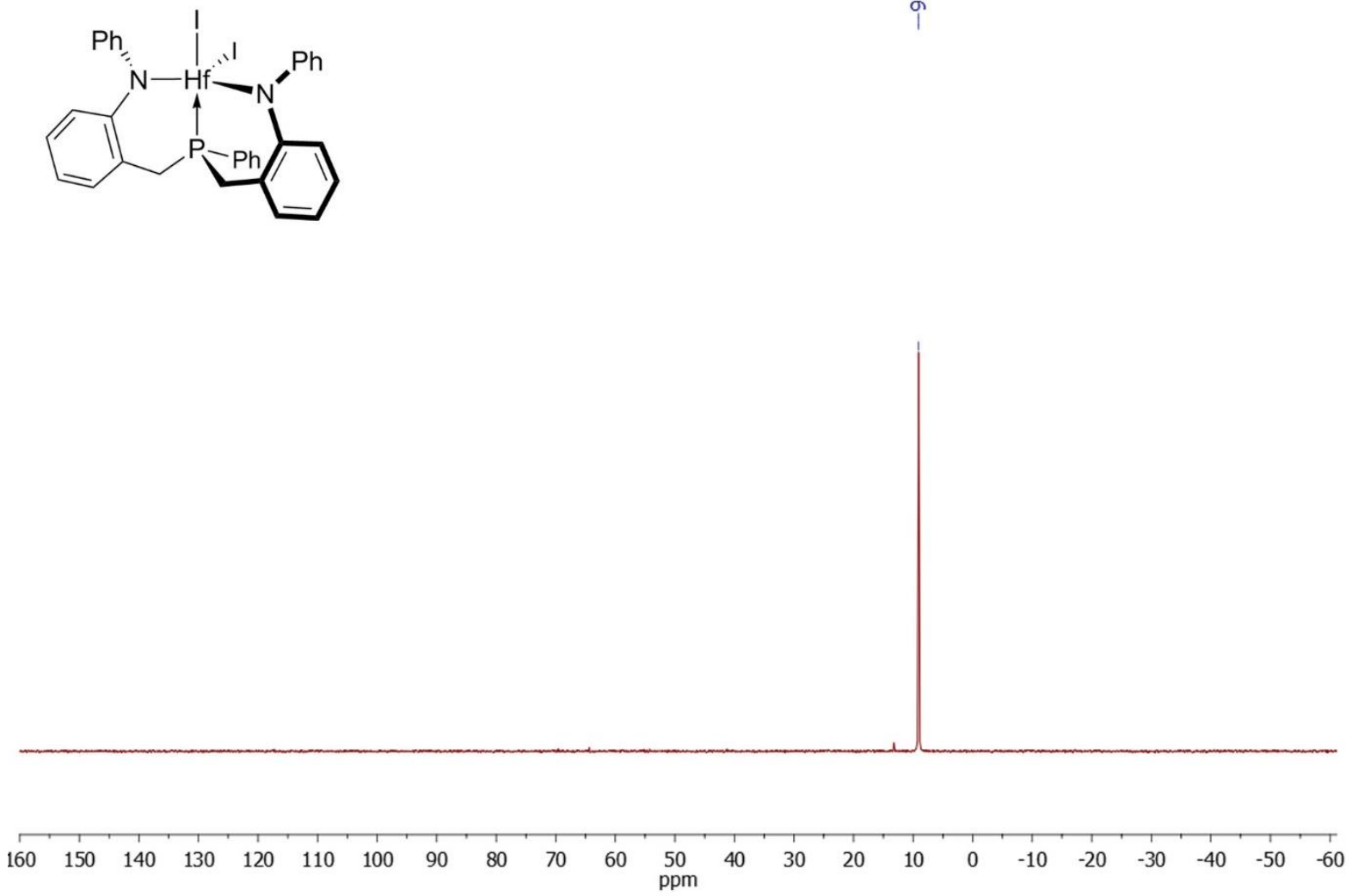

Fig. S17. ${ }^{31} \mathrm{P}\left\{{ }^{1} \mathrm{H}\right\}$ NMR $\left(243 \mathrm{MHz}, \mathrm{CD}_{2} \mathrm{Cl}_{2}\right)$ of $\left[{ }^{\mathrm{Ph}} \mathrm{A}\right]-\mathbf{2}-\mathrm{Hf}$.

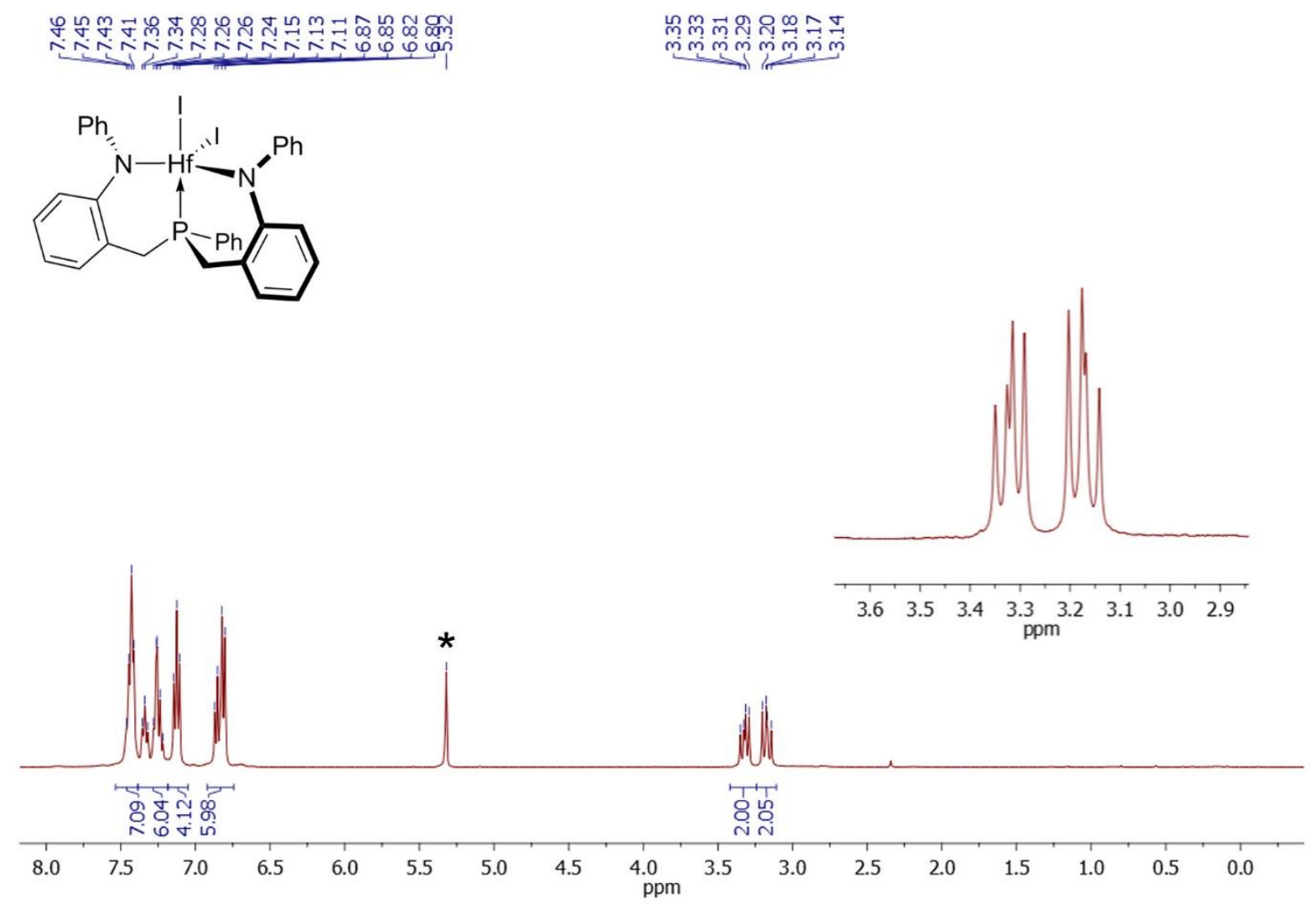

Fig. S18. ${ }^{1} \mathrm{H}$ NMR (400 MHz, $\mathrm{CD}_{2} \mathrm{Cl}_{2}$ ) of $\left[{ }^{\mathrm{Ph}} \mathrm{A}\right]-2-\mathrm{Hf}$ (residual proton signal of $\mathrm{CD}_{2} \mathrm{Cl}_{2}$ is labeled with $*$ ). 


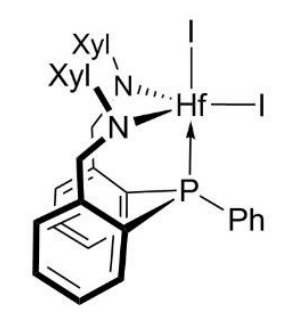

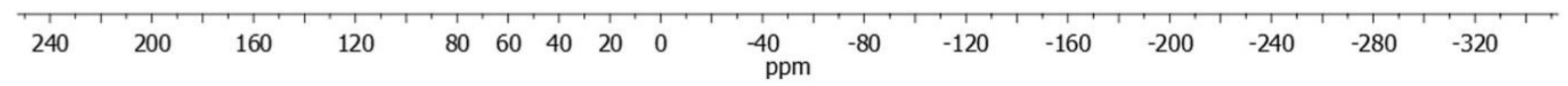

Fig. S19. ${ }^{31} \mathrm{P}\left\{{ }^{1} \mathrm{H}\right\} \mathrm{NMR}\left(243 \mathrm{MHz}, \mathrm{C}_{6} \mathrm{D}_{6}\right)$ of [B]-2-Hf.

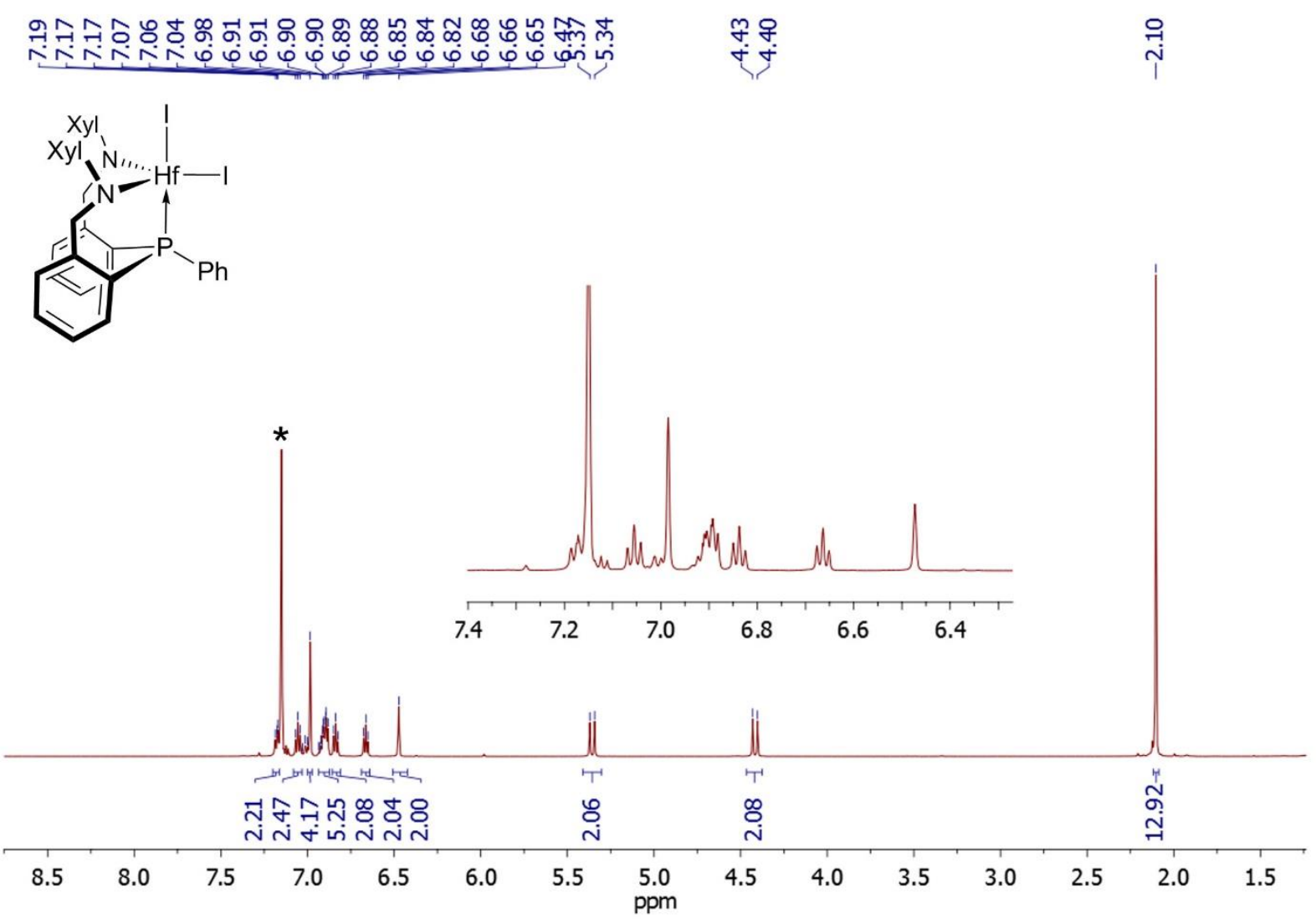

Fig. S20. ${ }^{1} \mathrm{H}$ NMR (600 MHz, $\left.\mathrm{C}_{6} \mathrm{D}_{6}\right)$ of [B]-2-Hf (residual proton signal of $\mathrm{C}_{6} \mathrm{D}_{6}$ is labeled with $*$ ). 

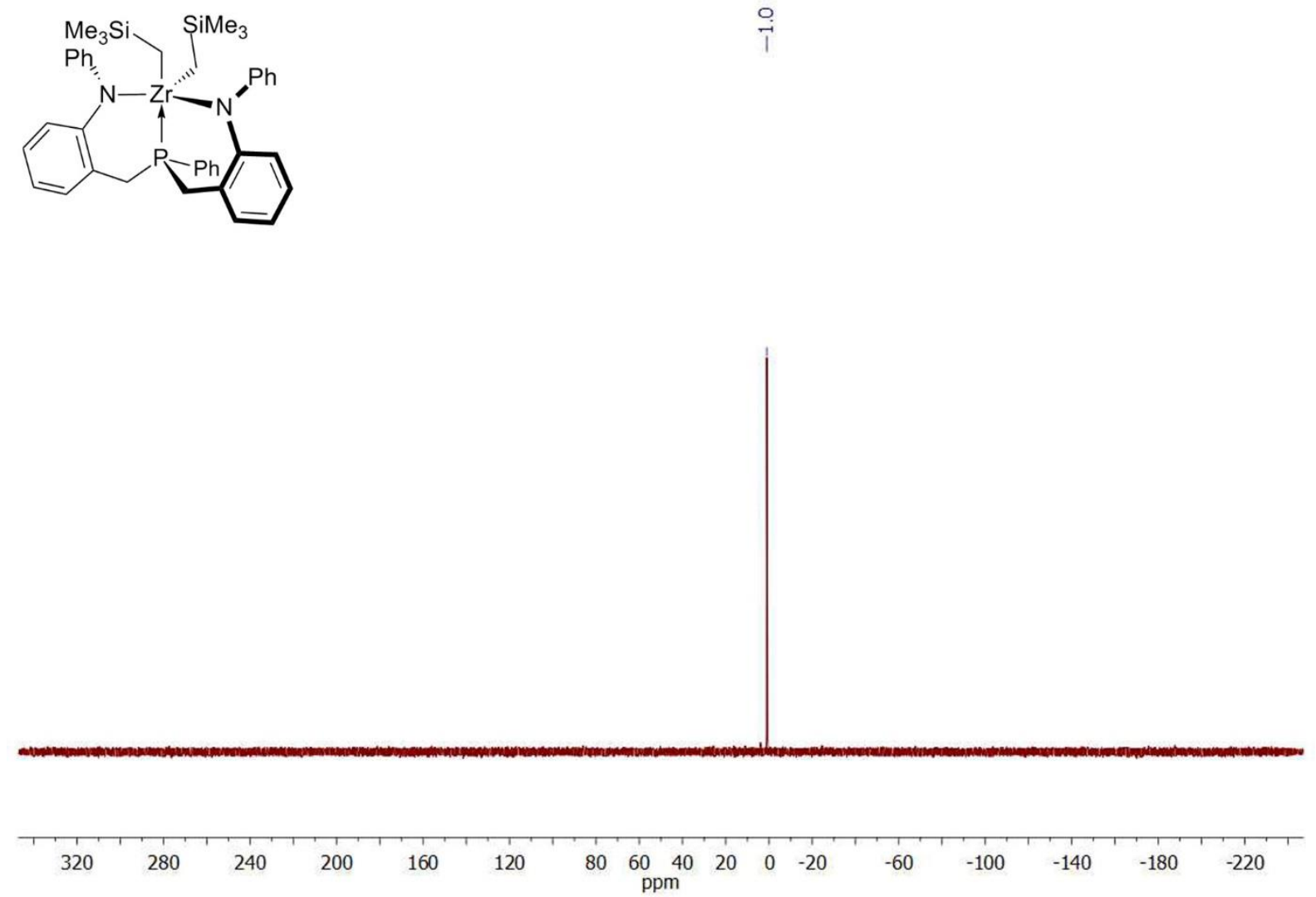

Fig. S21. ${ }^{31} \mathrm{P}\left\{{ }^{1} \mathrm{H}\right\} \mathrm{NMR}\left(243 \mathrm{MHz}, \mathrm{C}_{6} \mathrm{D}_{6}\right)$ of $\left[{ }^{\mathrm{Ph}} \mathrm{A}\right]-3-\mathrm{Zr}$.

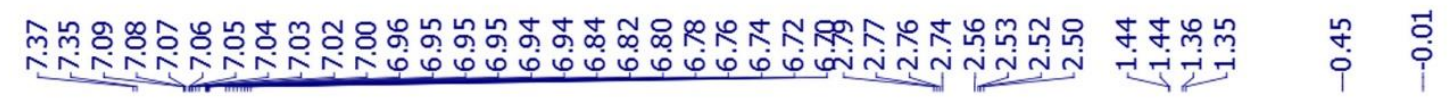

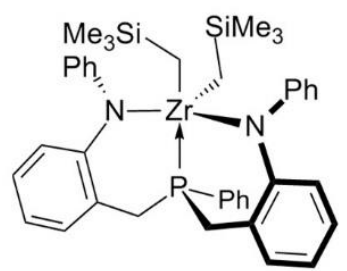

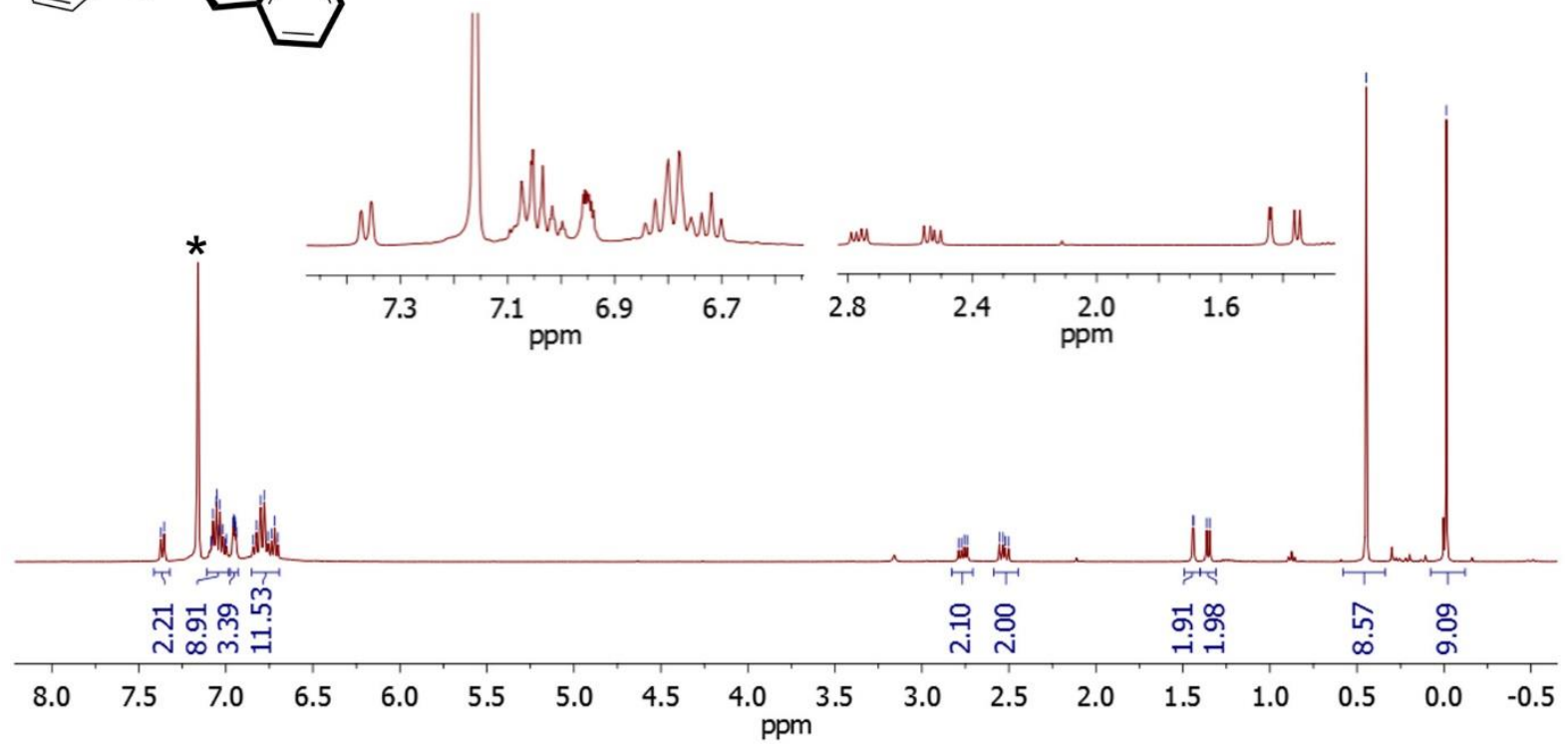

Fig. S22. ${ }^{1} \mathrm{H} N M R\left(600 \mathrm{MHz}, \mathrm{C}_{6} \mathrm{D}_{6}\right.$ ) of [ $\left.{ }^{\mathrm{Ph}} \mathrm{A}\right]-3-\mathrm{Zr}$ (residual proton signal of $\mathrm{C}_{6} \mathrm{D}_{6}$ is labeled with $*$ ). 


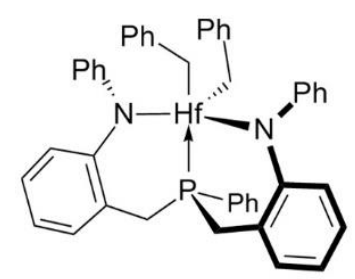

\begin{tabular}{|c|c|c|c|c|c|c|c|c|c|c|c|c|c|c|c|}
\hline 1111 & 11 & 11 & 11 & 11 & 1 & 1 & 111 & 1 & 1 & 11 & 1. & 11 & 19 & 1, & 1 \\
\hline 320 & 280 & 240 & 200 & 160 & 120 & 80 & $\begin{array}{c}60 \quad 40 \\
\mathrm{ppm}\end{array}$ & 20 & 0 & -20 & -60 & -100 & -140 & -180 & -220 \\
\hline
\end{tabular}

Fig. S23. ${ }^{31} \mathrm{P}\left\{{ }^{1} \mathrm{H}\right\} \mathrm{NMR}\left(243 \mathrm{MHz}, \mathrm{C}_{6} \mathrm{D}_{6}\right)$ of $\left[{ }^{\mathrm{Ph}} \mathrm{A}\right]-4-\mathrm{Hf}$.

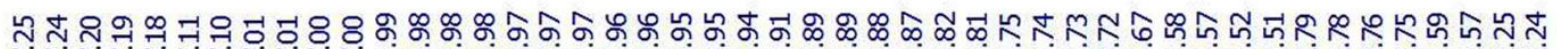

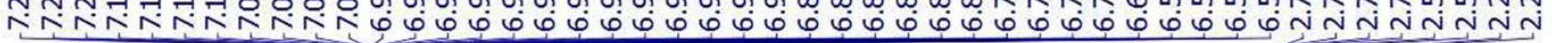

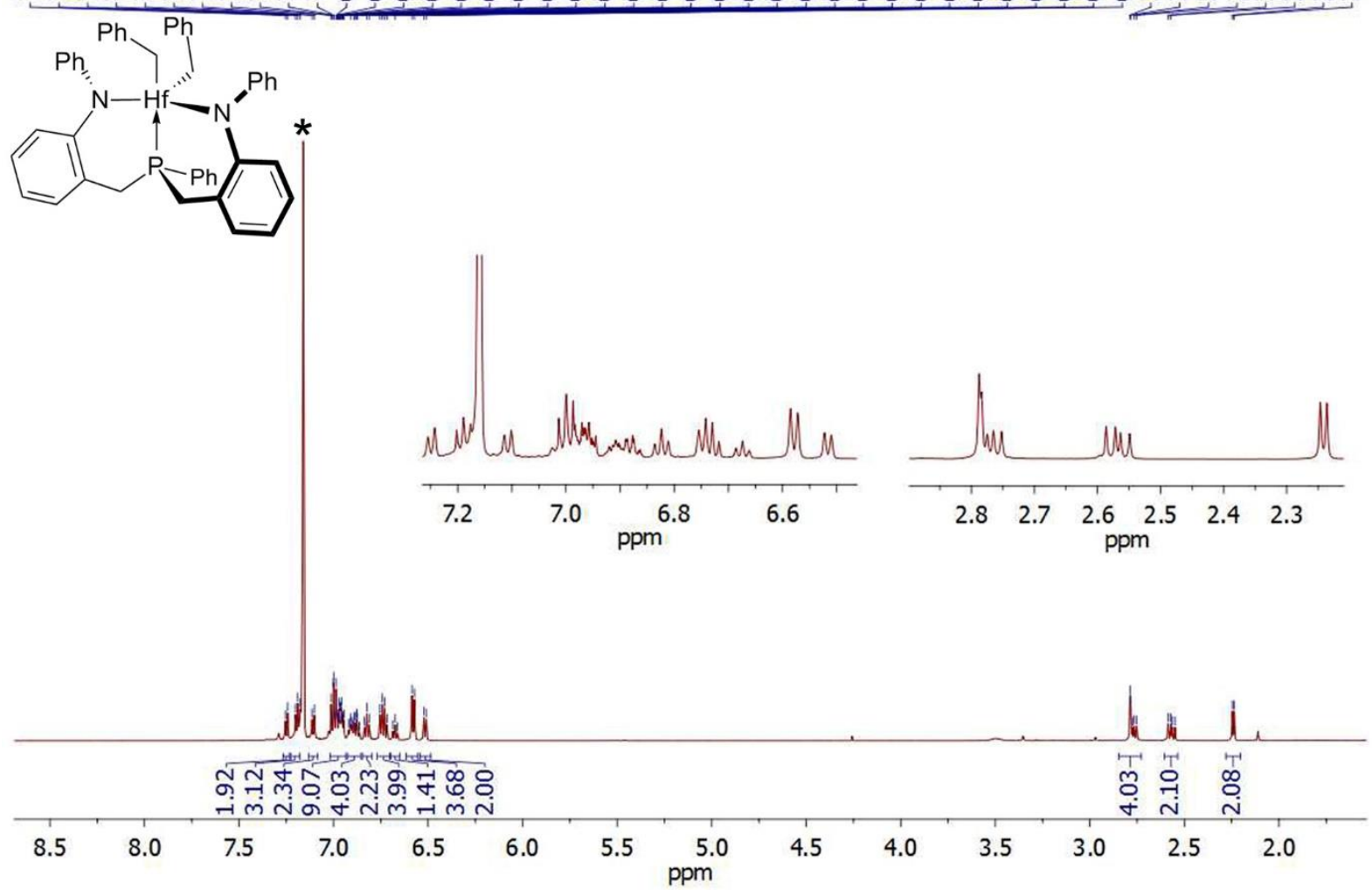

Fig. S24. ${ }^{1} \mathrm{H}$ NMR $\left(600 \mathrm{MHz}, \mathrm{C}_{6} \mathrm{D}_{6}\right)$ of $\left[{ }^{\mathrm{Ph}} \mathrm{A}\right]-4-\mathrm{Hf}$ (residual proton signal of $\mathrm{C}_{6} \mathrm{D}_{6}$ is labeled with $*$ ). 

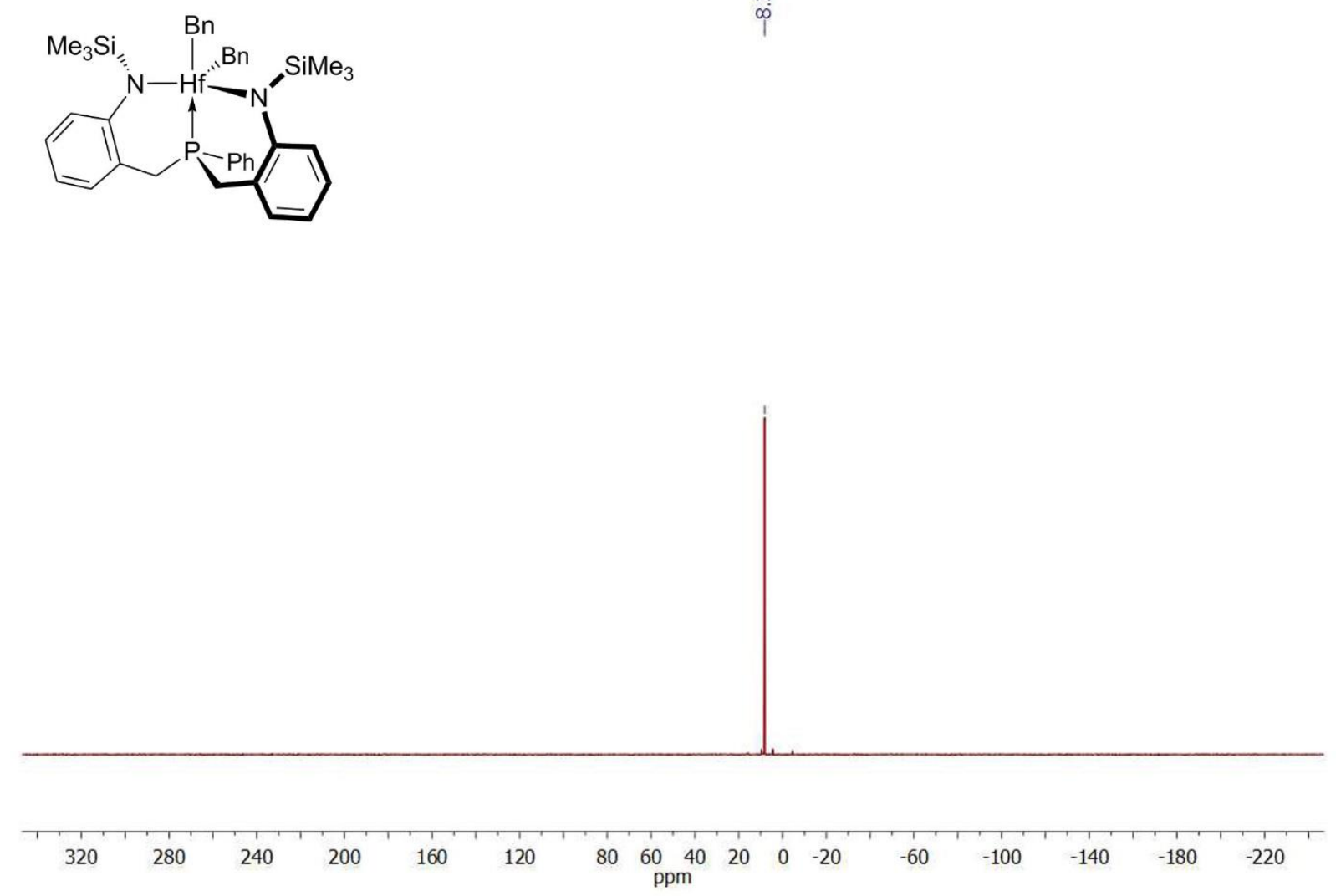

Fig. S25. ${ }^{31} \mathrm{P}\left\{{ }^{1} \mathrm{H}\right\} \mathrm{NMR}\left(243 \mathrm{MHz}, \mathrm{C}_{6} \mathrm{D}_{6}\right)$ of $\left[{ }^{\mathrm{Si}} \mathrm{A}\right]-4-\mathrm{Hf}\left(\mathrm{C}_{1}\right)$.

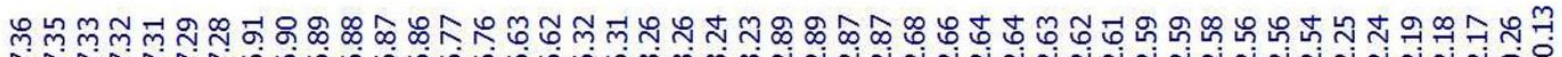

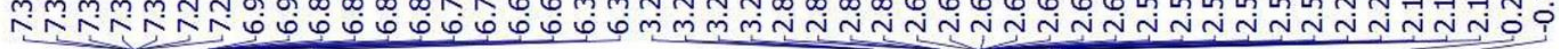

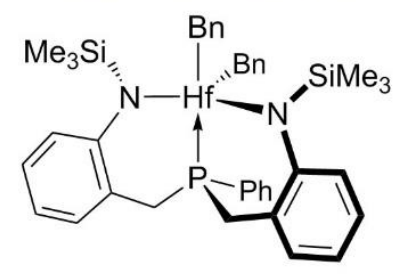
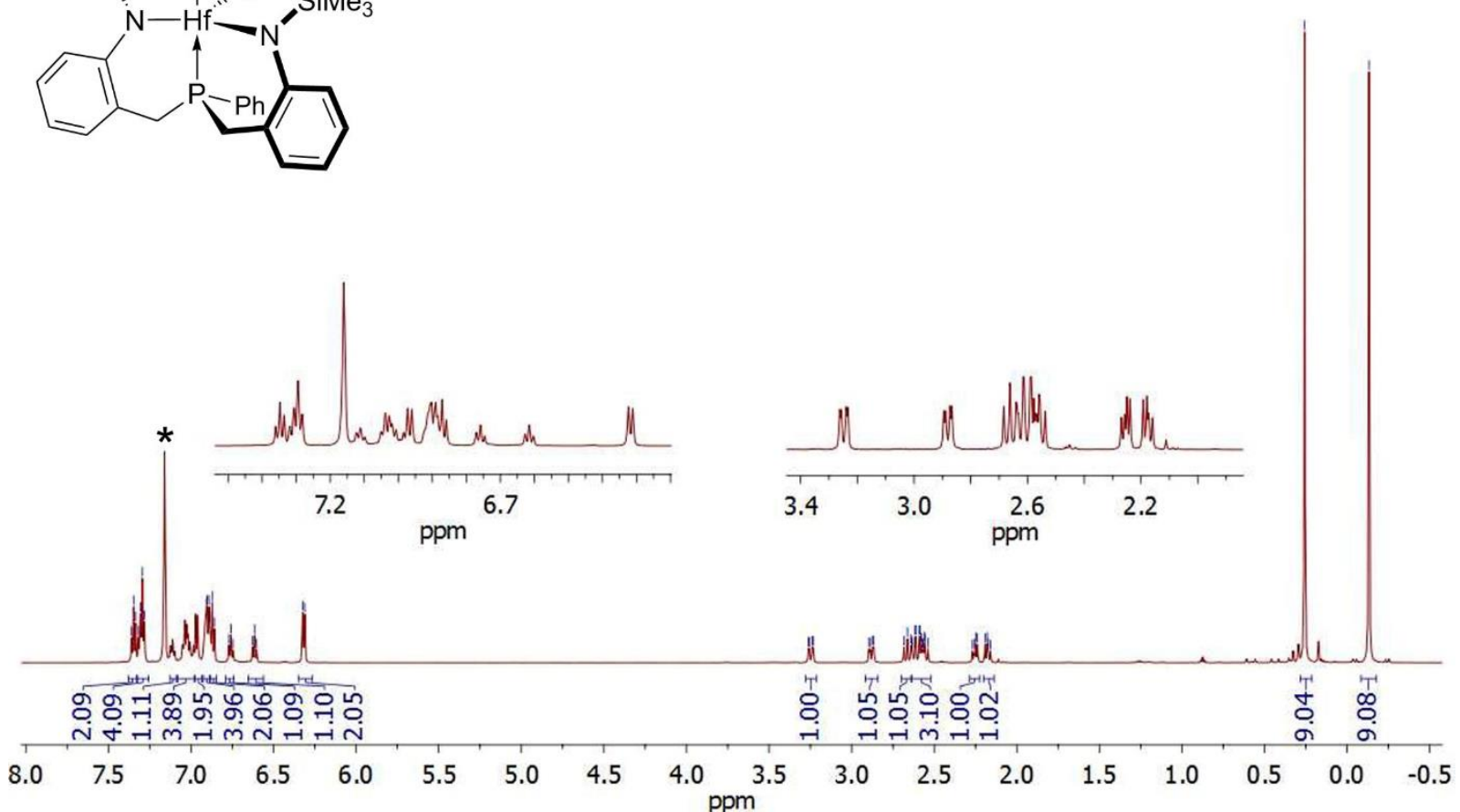

Fig. S26. ${ }^{1} \mathrm{H}$ NMR $\left(600 \mathrm{MHz}, \mathrm{C}_{6} \mathrm{D}_{6}\right)$ of $\left[{ }^{\mathrm{Si}} \mathrm{A}\right]-4-\mathrm{Hf}\left(\mathrm{C}_{1}\right)$ (residual proton signal of $\mathrm{C}_{6} \mathrm{D}_{6}$ is labeled with $*$ ). 

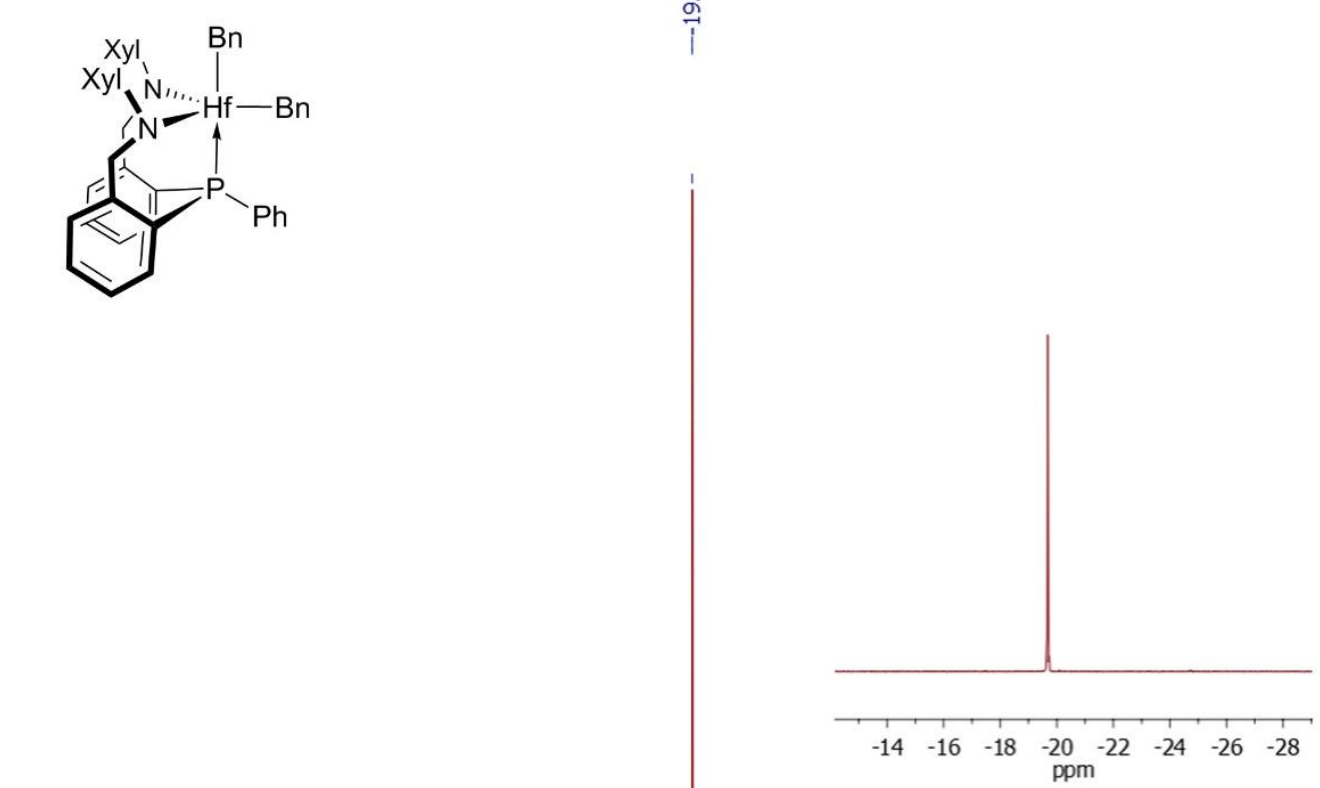

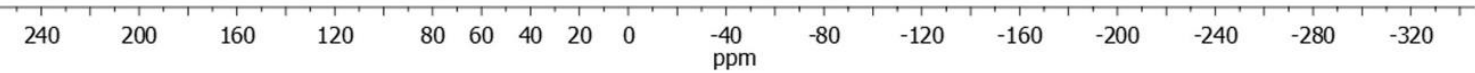

Fig. S27. ${ }^{31} \mathrm{P}\left\{{ }^{1} \mathrm{H}\right\}$ NMR $\left(243 \mathrm{MHz}, \mathrm{C}_{6} \mathrm{D}_{6}\right)$ of [B]-4-Hf.

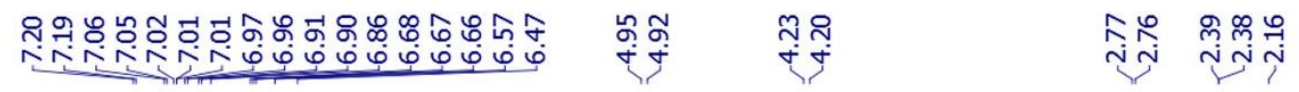
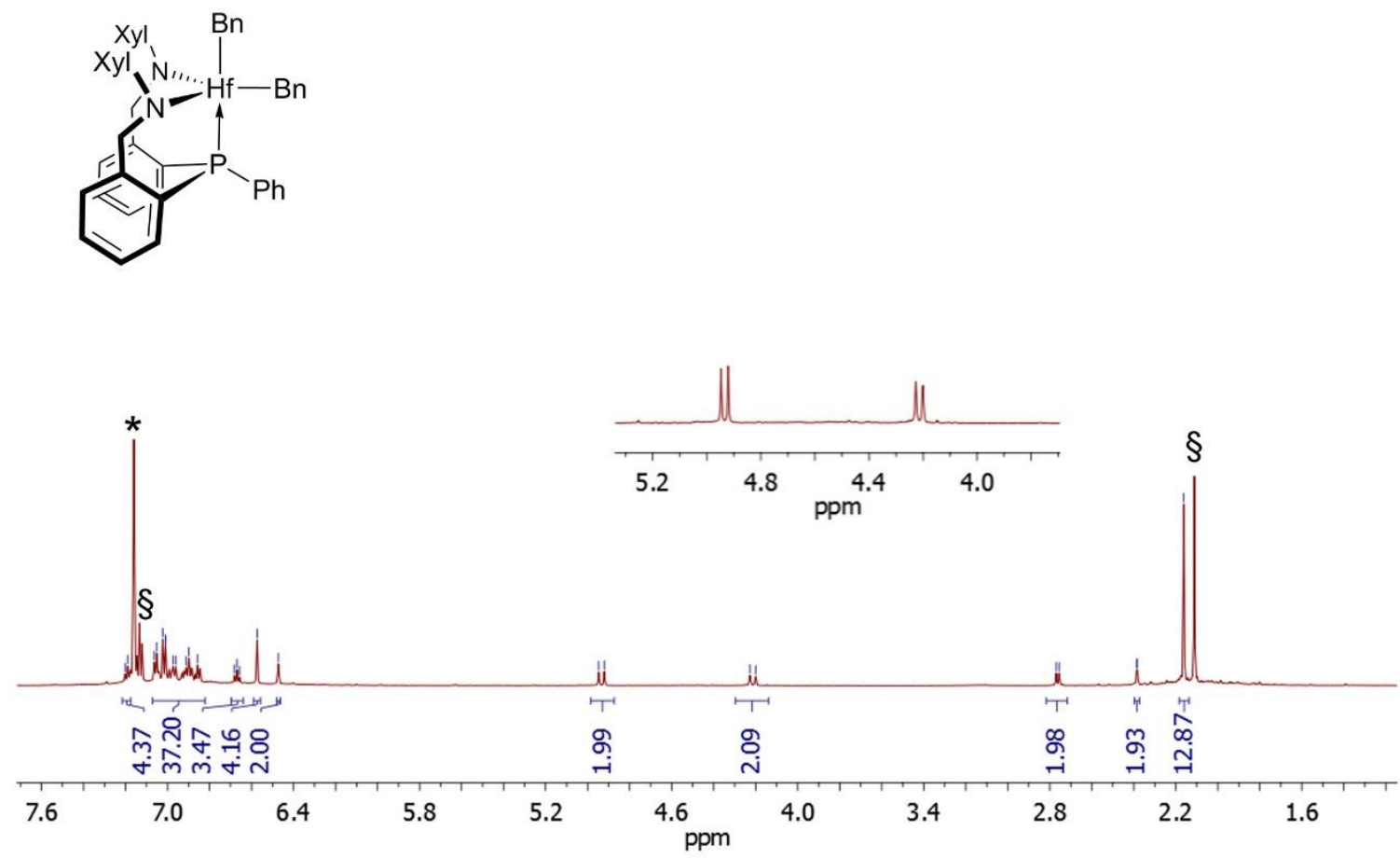

Fig. S28. ${ }^{1} \mathrm{H}$ NMR (600 MHz, $\mathrm{C}_{6} \mathrm{D}_{6}$ ) of [B]-4-Hf (residual proton signal of $\mathrm{C}_{6} \mathrm{D}_{6}$ is labeled with $*$, residual toluene signals are labeled with $\S)$. 


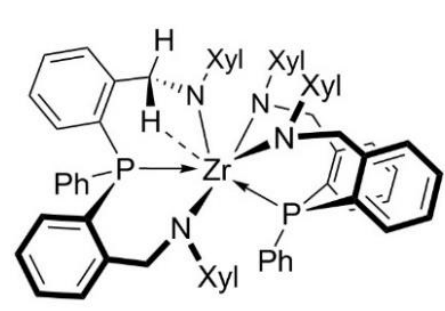

i

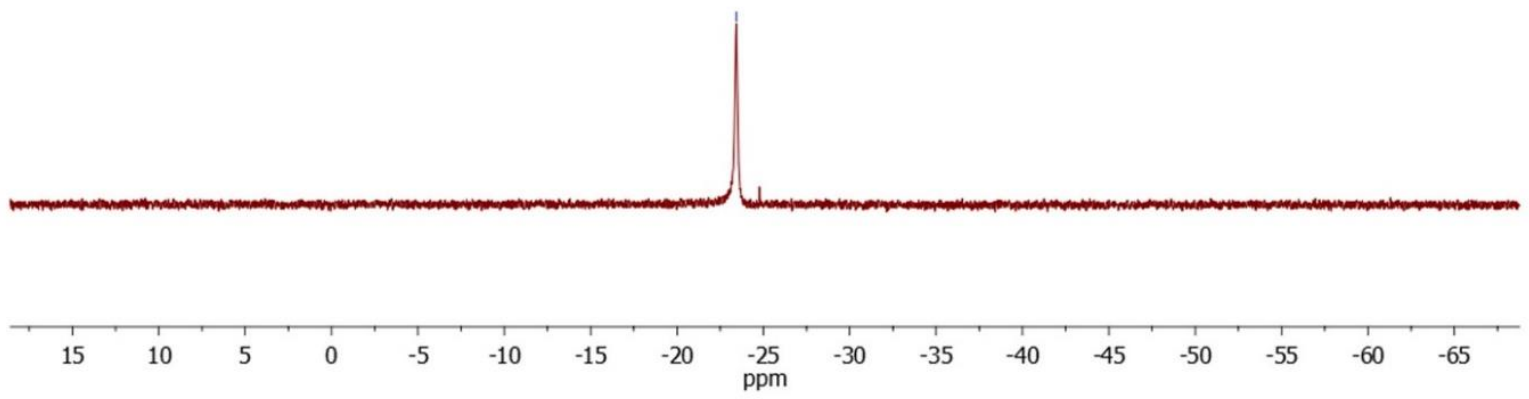

Fig. S29. ${ }^{31} \mathrm{P}\left\{{ }^{1} \mathrm{H}\right\}$ NMR $\left(162 \mathrm{MHz}, 373 \mathrm{~K}, \mathrm{Tol}-\mathrm{d}_{8}\right)$ of [B]-5-Zr.

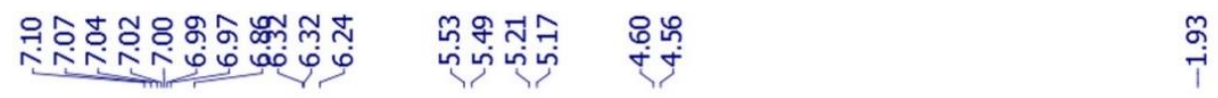

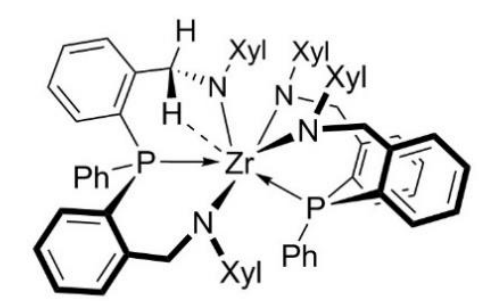

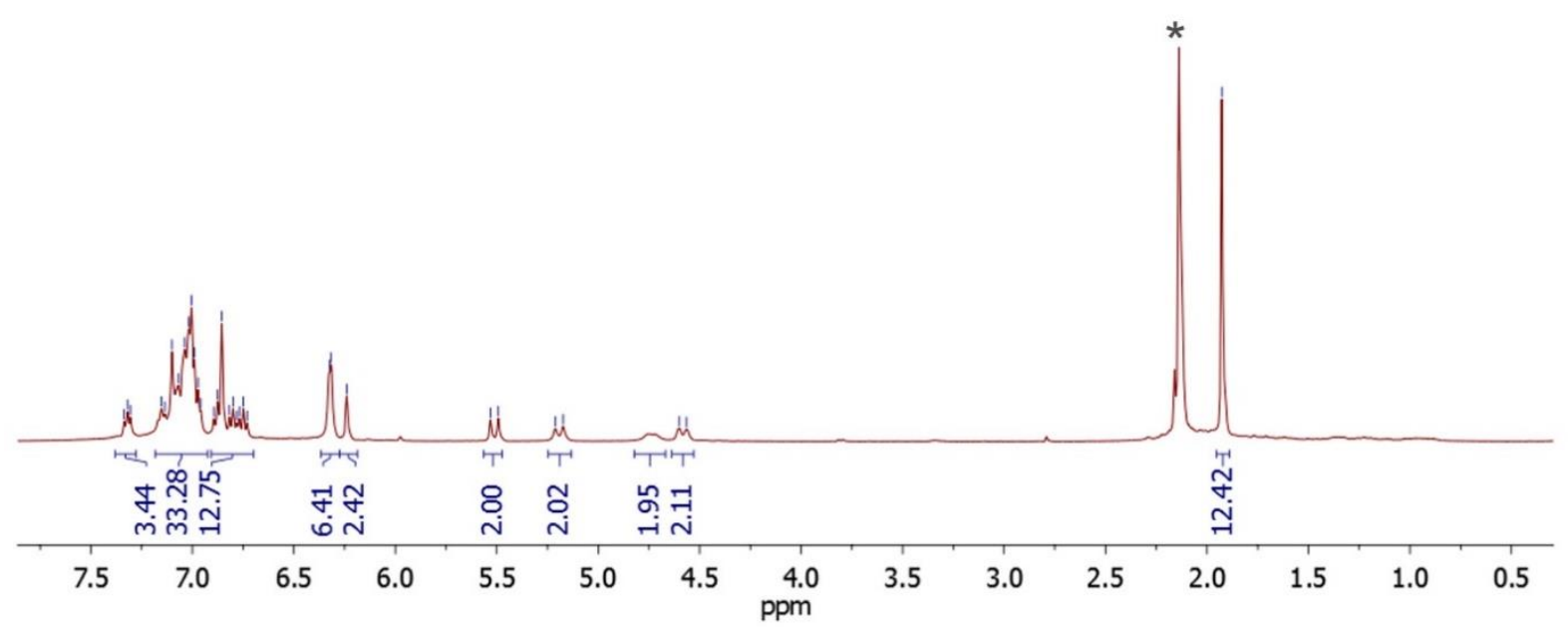

Fig. S30. ${ }^{1} \mathrm{H}$ NMR (400 MHz, $373 \mathrm{~K}$, Tol- $d_{8}$ ) of [B]-5-Zr (residual proton signal of Tol- $d_{8}$ is labeled with $*$ ). 


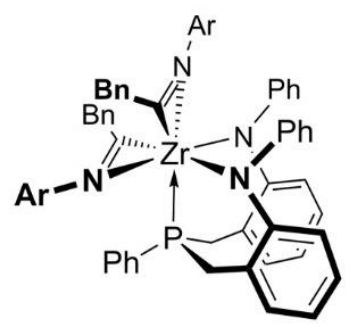

$\stackrel{\text { ก }}{\uparrow}$

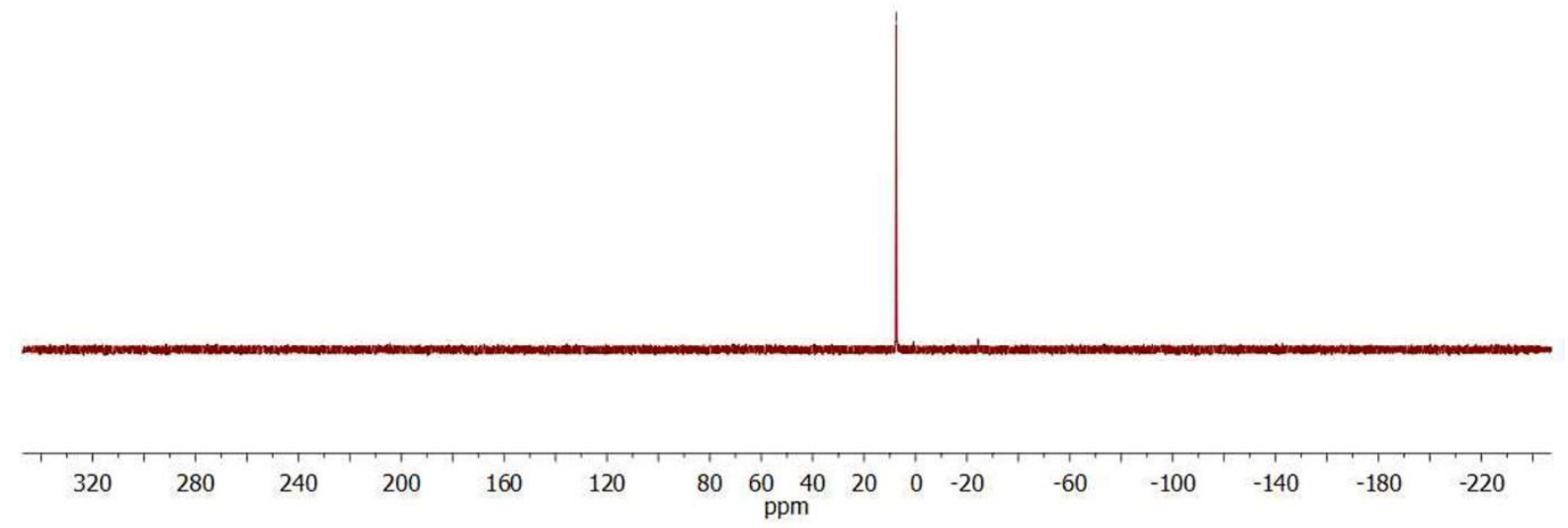

Fig. S31. ${ }^{31} \mathrm{P}\left\{{ }^{1} \mathrm{H}\right\}$ NMR $\left(243 \mathrm{MHz}, \mathrm{C}_{6} \mathrm{D}_{6}\right)$ of $\left[{ }^{\mathrm{Ph}} \mathrm{A}\right]-6-\mathrm{Zr}$.

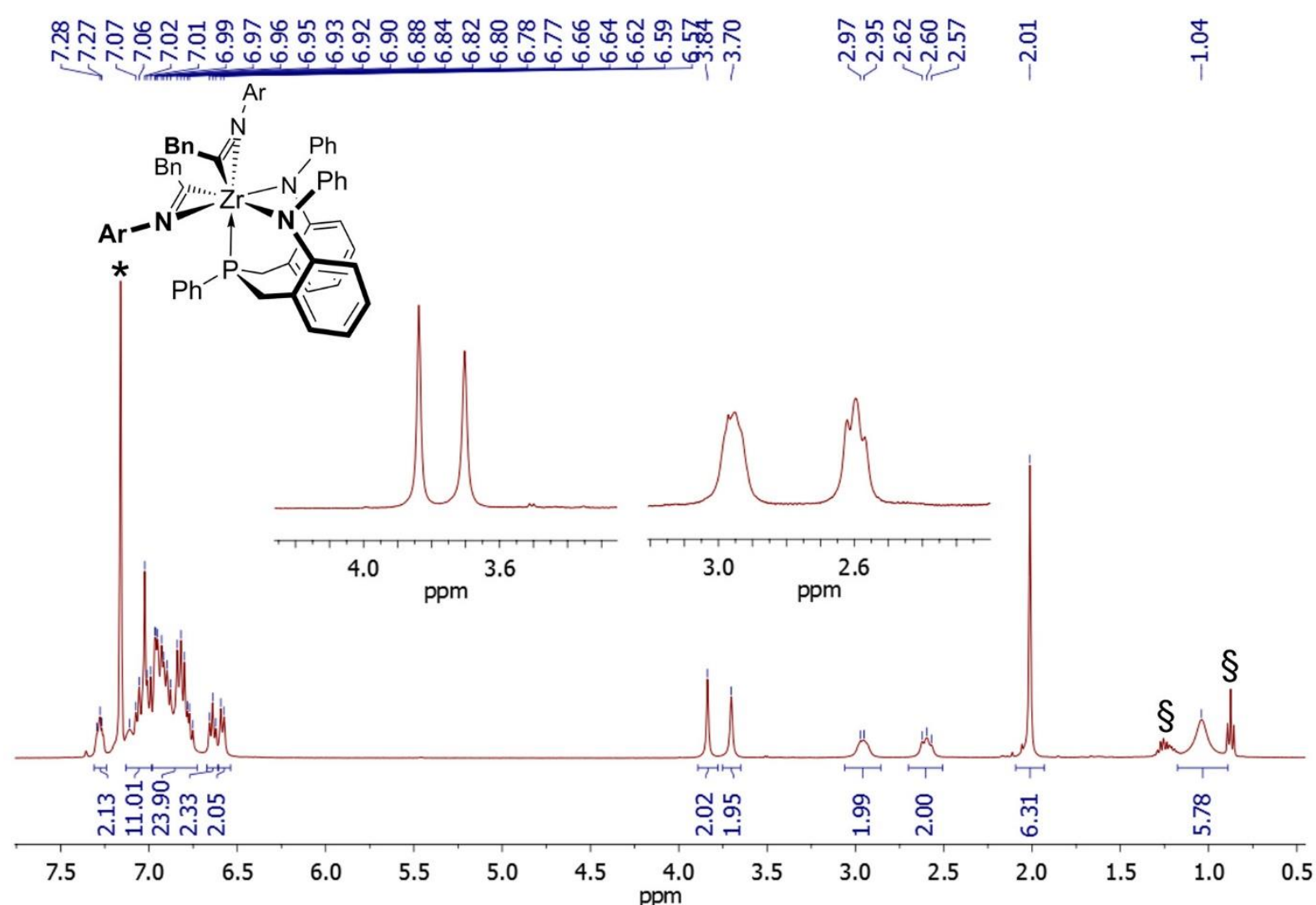

Fig. S32. ${ }^{1} \mathrm{H} N \mathrm{NMR}\left(600 \mathrm{MHz}, \mathrm{C}_{6} \mathrm{D}_{6}\right)$ of $\left[{ }^{\mathrm{Ph}} \mathrm{A}\right]-6-\mathrm{Zr}$ (residual proton signal of $\mathrm{C}_{6} \mathrm{D}_{6}$ is labeled with $*$, residual pentane signals are labeled with §). 


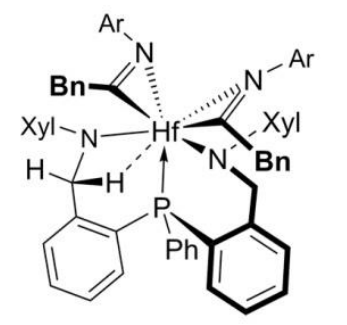

$\stackrel{\wedge}{i}$

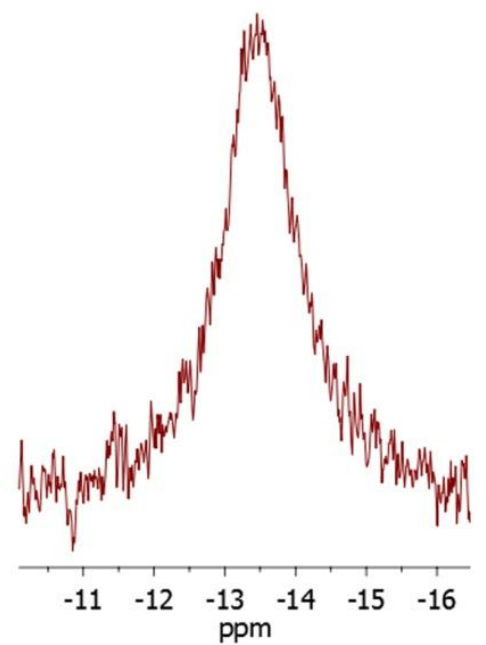

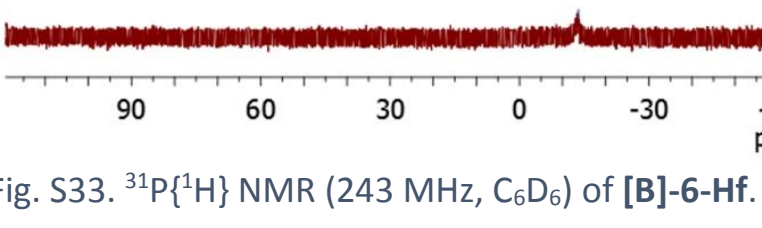
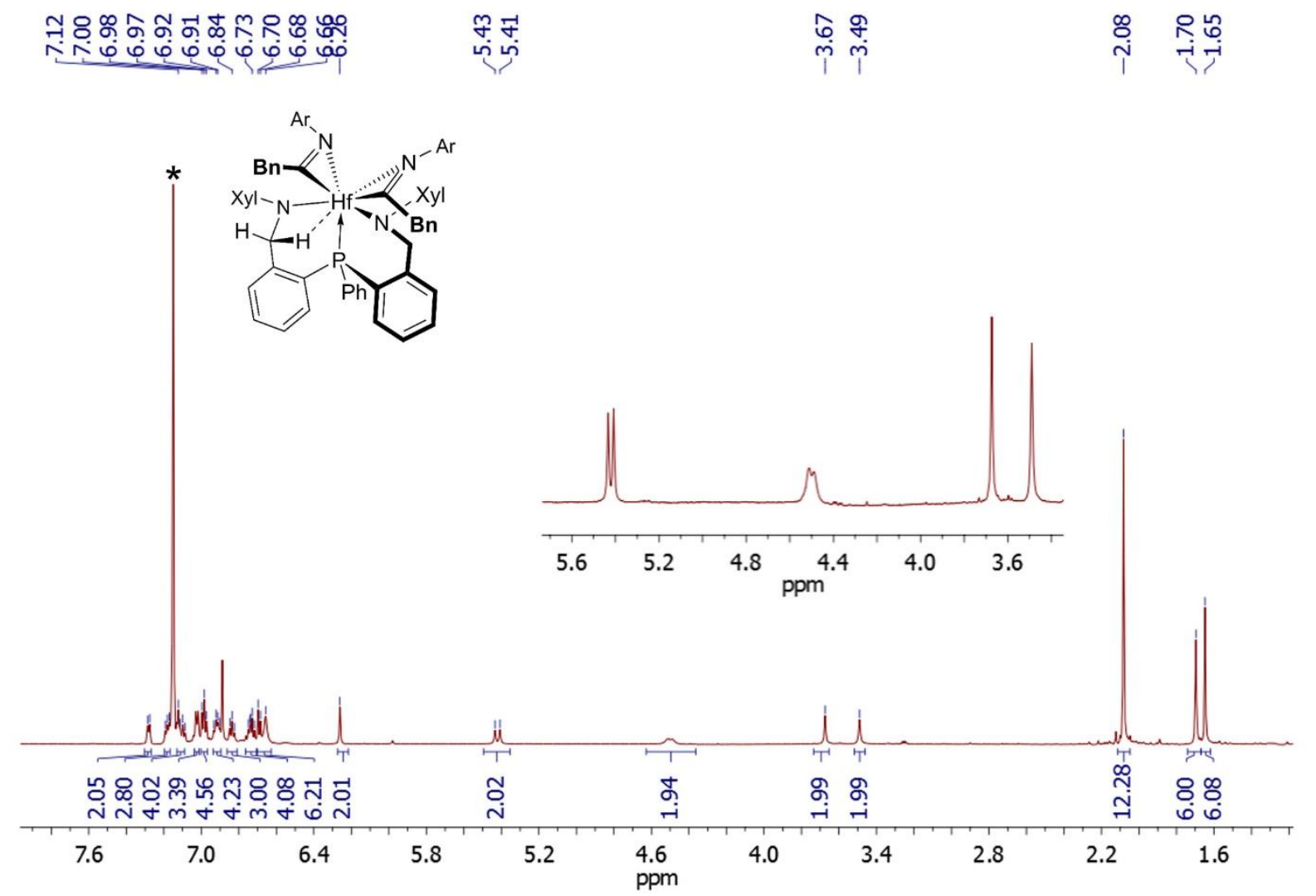

Fig. S34. ${ }^{1} \mathrm{H}$ NMR (600 MHz, $\left.\mathrm{C}_{6} \mathrm{D}_{6}\right)$ of [B]-6-Hf (residual proton signal of $\mathrm{C}_{6} \mathrm{D}_{6}$ is labeled with $*$ ). 


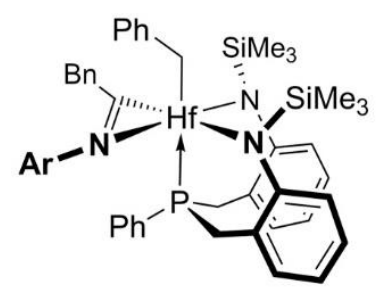

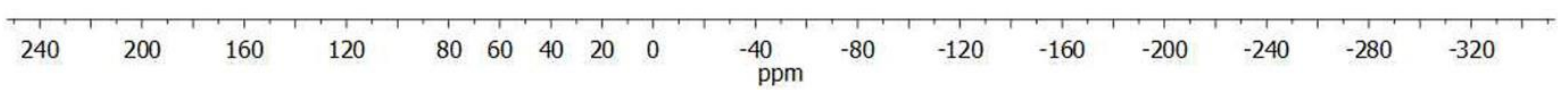

Fig. S35. ${ }^{31} \mathrm{P}\left\{{ }^{1} \mathrm{H}\right\} \mathrm{NMR}\left(243 \mathrm{MHz}, \mathrm{C}_{6} \mathrm{D}_{6}\right)$ of $\left[{ }^{\mathrm{Si}} \mathrm{A}\right]-6-\mathrm{Hf}$.

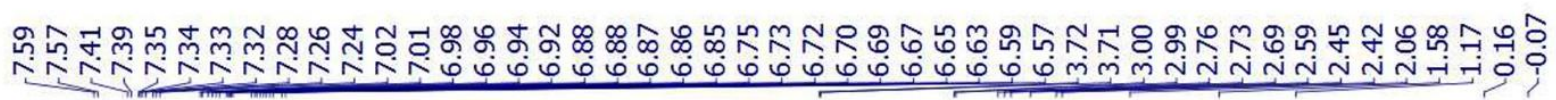<smiles>CN(C)C(Cc1ccccc1)(Cc1ccccc1)N(C)CCc1ccccc1</smiles>

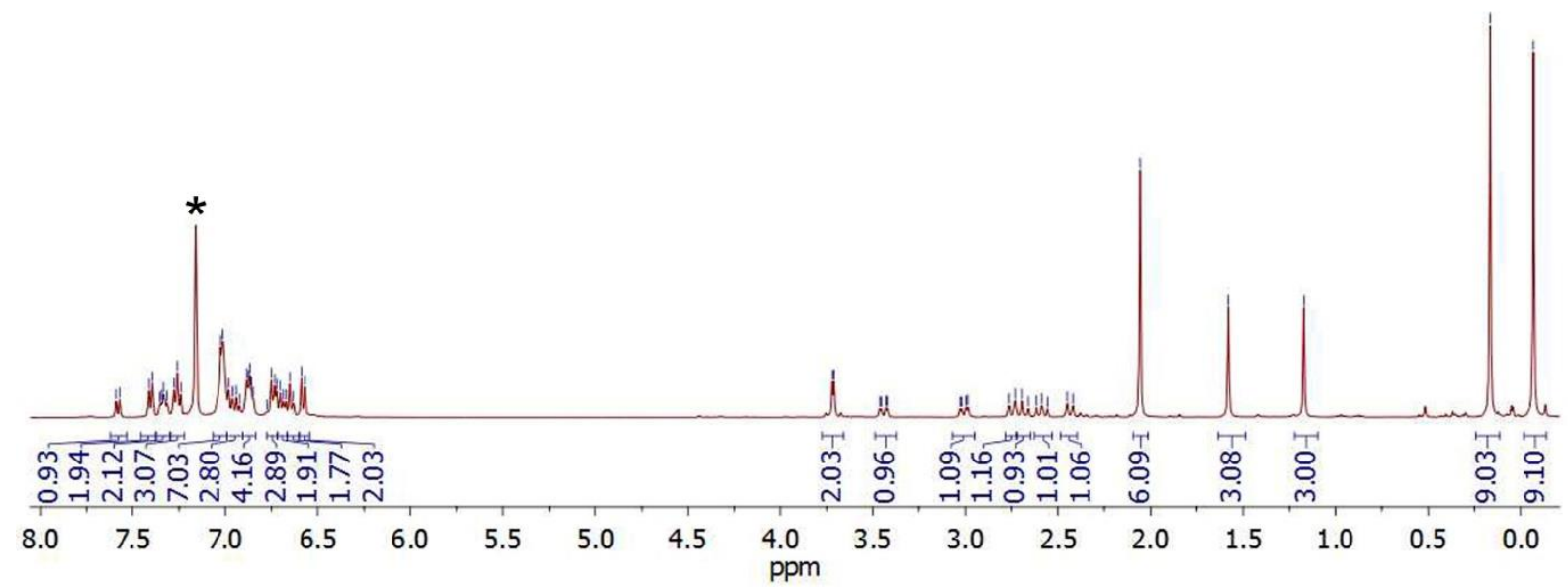

Fig. S36. ${ }^{1} \mathrm{H} N M R\left(600 \mathrm{MHz}, \mathrm{C}_{6} \mathrm{D}_{6}\right)$ of $\left[{ }^{\mathrm{Si}} \mathrm{A}\right]-6-\mathrm{Hf}$ (residual proton signal of $\mathrm{C}_{6} \mathrm{D}_{6}$ is labeled with $*$ ). 


\section{4) Crystallographic Data}

Table S1: Details of the crystal structure determinations of $[\mathbf{B}] \mathrm{H}_{2}$ and $\left[{ }^{\mathrm{Ph}} \mathbf{A}\right]-\mathbf{- 1}-\mathbf{Z r}$.

\begin{tabular}{|c|c|c|}
\hline compound & {$[B] \mathrm{H}_{2}$} & {$\left[{ }^{\mathrm{Ph}} \mathrm{A}\right]-1-\mathrm{Zr}$} \\
\hline formula & $\mathrm{C}_{36} \mathrm{H}_{37} \mathrm{~N}_{2} \mathrm{P}$ & $\mathrm{C}_{36} \mathrm{H}_{39} \mathrm{~N}_{4} \mathrm{PZr}$ \\
\hline$M_{\mathrm{r}}$ & 528.64 & 649.90 \\
\hline crystal system & monoclinic & triclinic \\
\hline space group & $P 2_{1} / n$ & $P-1$ \\
\hline$a / \AA$ & $17.735(11)$ & $8.975(4)$ \\
\hline$b / \AA$ & $12.421(7)$ & $11.713(5)$ \\
\hline$c / \AA$ & 27.719(17) & $16.176(6)$ \\
\hline$\alpha /^{\circ}$ & & $81.466(14)$ \\
\hline$\beta /^{\circ}$ & $106.880(16)$ & $89.451(6)$ \\
\hline$\gamma /{ }^{\circ}$ & & $70.647(8)$ \\
\hline$V / \AA^{3}$ & $5843(6)$ & $1585.2(11)$ \\
\hline$Z$ & 8 & 2 \\
\hline$F_{000}$ & 2256 & 676 \\
\hline$d_{c} / \mathrm{Mg} \cdot \mathrm{m}^{-3}$ & 1.202 & 1.362 \\
\hline X-radiation, $\lambda / \AA$ & Mo- $K_{\alpha}, 0.71073$ & Mo- $K_{\alpha}, 0.71073$ \\
\hline$\mu / \mathrm{mm}^{-1}$ & 0.121 & 0.429 \\
\hline max., min. transmission factors & $0.7453,0.6803^{a}$ & $0.7464,0.7130^{a}$ \\
\hline data collect. temperature $/ \mathrm{K}$ & $100(1)$ & $100(1)$ \\
\hline$\theta$ range $/^{\circ}$ & $1.22-25.81$ & $2.41-32.47$ \\
\hline index ranges (indep. set) $h, k, l$ & $-20 \ldots 21, \pm 15, \pm 33$ & $\pm 13, \pm 17, \pm 24$ \\
\hline reflections measured & 95088 & 40098 \\
\hline unique $\left[R_{\text {int }}\right]$ & $11180[0.0877]$ & $10696[0.0210]$ \\
\hline observed $[I \geq 2 \sigma(I)]$ & 7656 & 9901 \\
\hline parameters refined [restraints] & $723[0]$ & $468[0]$ \\
\hline GooF on $F^{2}$ & 1.005 & 1.040 \\
\hline$R$ indices $[F>4 \sigma(F)] R(F), w R\left(F^{2}\right)$ & $0.0492,0.1088$ & $0.0244,0.0630$ \\
\hline$R$ indices (all data) $R(F), w R\left(F^{2}\right)$ & $0.0863,0.1262$ & $0.0273,0.0653$ \\
\hline difference density: $\max , \min / \mathrm{e} \cdot \AA^{-3}$ & $0.052,-0.271$ & $0.742,-0.212$ \\
\hline
\end{tabular}

a empirical absorption correction 
Table S2: Details of the crystal structure determinations of [B]-1-Zr·0.5 pentane and [B]-1-Hf·0.5 $\mathrm{Et}_{2} \mathrm{O}$.

\begin{tabular}{|c|c|c|}
\hline compound & [B]-1-Zr-0.5 pentane & [B]-1-Hf-0.5 $\mathrm{Et}_{2} \mathrm{O}$ \\
\hline formula & $\mathrm{C}_{42.5} \mathrm{H}_{53} \mathrm{~N}_{4} \mathrm{PZr}$ & $\mathrm{C}_{42.5} \mathrm{H}_{53} \mathrm{HfN}_{4} \mathrm{P}$ \\
\hline$M_{\mathrm{r}}$ & 742.08 & 829.35 \\
\hline crystal system & monoclinic & triclinic \\
\hline space group & $C 2 / c$ & $P-1$ \\
\hline$a / \AA$ & $18.9485(3)$ & $11.336(5)$ \\
\hline$b / \AA$ & $13.7692(2)$ & $13.390(5)$ \\
\hline$c / \AA$ & $30.4136(5)$ & $14.599(5)$ \\
\hline$\alpha /^{\circ}$ & & $102.302(13)$ \\
\hline$\beta /^{\circ}$ & $105.8995(17)$ & $102.584(12)$ \\
\hline$\gamma /{ }^{\circ}$ & & $110.230(10)$ \\
\hline$V / \AA^{3}$ & $7631.5(2)$ & 1926.1(13) \\
\hline$z$ & 8 & 2 \\
\hline$F_{000}$ & 3128 & 846 \\
\hline$d_{c} / \mathrm{Mg} \cdot \mathrm{m}^{-3}$ & 1.292 & 1.430 \\
\hline X-radiation, $\lambda / \AA$ & Mo- $K_{\alpha}, 0.71073$ & Mo- $K_{\alpha}, 0.71073$ \\
\hline$\mu / \mathrm{mm}^{-1}$ & 0.365 & 2.784 \\
\hline max., min. transmission factors & $0.976,0.952^{a}$ & $0.5655,0.5064^{b}$ \\
\hline data collect. temperature $/ \mathrm{K}$ & $120(1)$ & $100(1)$ \\
\hline$\theta$ range $/^{\circ}$ & $3.27-32.86$ & $2.02-32.42$ \\
\hline index ranges (indep. set) $h, k, l$ & $-27 \ldots 28, \pm 20, \pm 45$ & $-17 \ldots 16 \pm 19, \pm 21$ \\
\hline reflections measured & 114232 & 49818 \\
\hline unique $\left[R_{\text {int }}\right]$ & $13469[0.0828]$ & $12933[0.0315]$ \\
\hline observed $[I \geq 2 \sigma(I)]$ & 10690 & 11897 \\
\hline parameters refined [restraints] & $447[0]$ & $423[0]$ \\
\hline GooF on $F^{2}$ & 1.071 & 1.077 \\
\hline$R$ indices $[F>4 \sigma(F)] R(F), w R\left(F^{2}\right)$ & $0.0484,0.1027$ & $0.0217,0.0484$ \\
\hline$R$ indices (all data) $R(F), w R\left(F^{2}\right)$ & $0.0681,0.1103$ & $0.0259,0.0501$ \\
\hline difference density: $\max , \min / e \cdot \AA^{-3}$ & $1.375,-0.810$ & $1.679,-0.767$ \\
\hline
\end{tabular}

a numerical absorption correction. ${ }^{b}$ empirical absorption correction. 
Table S3: Details of the crystal structure determinations of [ $\left.{ }^{\mathrm{Ph}} \mathrm{A}\right]-\mathbf{1 - T i}$ and [B]-1-Ti-solv.

\begin{tabular}{|c|c|c|}
\hline compound & {$\left[{ }^{\mathrm{Ph}} \mathrm{A}\right]-1-\mathrm{Ti}$} & [B]-1-Ti-solv \\
\hline formula & $\mathrm{C}_{36} \mathrm{H}_{39} \mathrm{~N}_{4} \mathrm{PTi}$ & $\mathrm{C}_{42} \mathrm{H}_{52} \mathrm{~N}_{4} \mathrm{O}_{0.5} \mathrm{PTi}$ \\
\hline$M_{\mathrm{r}}$ & 606.58 & 699.74 \\
\hline crystal system & triclinic & triclinic \\
\hline space group & $P-1$ & $P-1$ \\
\hline$a / \AA$ & $9.060(5)$ & $11.32651(18)$ \\
\hline$b / \AA$ & $11.614(5)$ & $13.3144(2)$ \\
\hline$c / \AA$ & $15.921(7)$ & $14.6393(3)$ \\
\hline$\alpha /^{\circ}$ & $81.263(16)$ & $102.6184(16)$ \\
\hline$\beta /^{\circ}$ & $89.363(8)$ & $102.3519(15)$ \\
\hline$\gamma /{ }^{\circ}$ & $70.231(9)$ & $110.2763(15)$ \\
\hline$V / \AA^{3}$ & $1556.7(12)$ & $1917.05(6)$ \\
\hline$Z$ & 2 & 2 \\
\hline$F_{000}$ & 640 & 746 \\
\hline$d_{c} / \mathrm{Mg} \cdot \mathrm{m}^{-3}$ & 1.294 & 1.212 \\
\hline X-radiation, $\lambda / \AA$ & Mo- $K_{\alpha}, 0.71073$ & $\mathrm{Cu}-K_{\alpha}, 1.54184$ \\
\hline$\mu / \mathrm{mm}^{-1}$ & 0.358 & 2.549 \\
\hline max., min. transmission factors & $0.7464,0.7057^{a}$ & $0.846,0.721^{b}$ \\
\hline data collect. temperature $/ \mathrm{K}$ & $100(1)$ & $120(1)$ \\
\hline$\theta$ range $/^{\circ}$ & $1.30-32.51$ & $3.74-70.75$ \\
\hline index ranges (indep. set) $h, k, I$ & $\pm 13, \pm 17, \pm 23$ & $\pm 13,-16 \ldots 15,-17 \ldots 15$ \\
\hline reflections measured & 40455 & 46804 \\
\hline unique $\left[R_{\text {int }}\right]$ & $10480[0.0419]$ & $7290[0.0245]$ \\
\hline observed $[I \geq 2 \sigma(I)]$ & 8253 & 6888 \\
\hline parameters refined [restraints] & $468[0]$ & $423[0]$ \\
\hline GooF on $F^{2}$ & 1.037 & 1.044 \\
\hline$R$ indices $[F>4 \sigma(F)] R(F), w R\left(F^{2}\right)$ & $0.0412,0.0945$ & $0.0291,0.0784$ \\
\hline$R$ indices (all data) $R(F), w R\left(F^{2}\right)$ & $0.0599,0.1034$ & $0.0311,0.0795$ \\
\hline difference density: $\max , \min / \mathrm{e} \cdot \AA^{-3}$ & $0.523,-0.351$ & $0.295,-0.344$ \\
\hline
\end{tabular}

${ }^{a}$ empirical absorption correction. ${ }^{\mathrm{b}}$ numerical absorption correction. 
Table S4: Details of the crystal structure determinations of $\left[{ }^{\mathrm{Ph}} \mathrm{A}\right]-\mathbf{2}-\mathrm{Ti}$ and $[\mathrm{B}]-\mathbf{2}-\mathrm{Ti} \cdot$ toluene.

\begin{tabular}{|c|c|c|}
\hline compound & {$\left[{ }^{\mathrm{Ph}} \mathrm{A}\right]-\mathbf{2}-\mathrm{Ti}$} & [B]-2-Ti-toluene \\
\hline formula & $\mathrm{C}_{32} \mathrm{H}_{27} \mathrm{l}_{2} \mathrm{~N}_{2} \mathrm{PTi}$ & $\mathrm{C}_{43} \mathrm{H}_{43} \mathrm{I}_{2} \mathrm{~N}_{2} \mathrm{PTi}$ \\
\hline$M_{\mathrm{r}}$ & 772.22 & 920.46 \\
\hline crystal system & monoclinic & monoclinic \\
\hline space group & $P 2_{1} / c$ & $P 2_{1} / c$ \\
\hline$a / \AA ̊$ & $11.75732(8)$ & $12.83406(11)$ \\
\hline$b / \AA$ & $14.56140(10)$ & $13.50260(12)$ \\
\hline$c / \AA$ & $17.37463(12)$ & $22.40089(18)$ \\
\hline$\beta /^{\circ}$ & $102.2165(7)$ & $96.3526(8)$ \\
\hline$V / \AA^{3}$ & $2907.23(3)$ & $3858.09(6)$ \\
\hline$Z$ & 4 & 4 \\
\hline$F_{000}$ & 1504 & 1832 \\
\hline$d_{c} / \mathrm{Mg} \cdot \mathrm{m}^{-3}$ & 1.764 & 1.585 \\
\hline X-radiation, $\lambda / \AA$ & Mo- $K_{\alpha}, 0.71073$ & Mo- $K_{\alpha}, 0.71073$ \\
\hline$\mu / \mathrm{mm}^{-1}$ & 2.498 & 1.896 \\
\hline max., min. transmission factors & $0.883,0.769^{a}$ & $0.886,0.768^{a}$ \\
\hline data collect. temperature $/ \mathrm{K}$ & $120(1)$ & $120(1)$ \\
\hline$\theta$ range $/^{\circ}$ & $3.01-32.36$ & $3.38-28.83$ \\
\hline index ranges (indep. set) $h, k, I$ & $\pm 17, \pm 21,-25 \ldots 26$ & $\pm 17, \pm 18, \pm 30$ \\
\hline reflections measured & 87824 & 173153 \\
\hline unique $\left[R_{\text {int }}\right]$ & $10053[0.0297]$ & $9858[0.0578]$ \\
\hline observed $[I \geq 2 \sigma(I)]$ & 9262 & 8561 \\
\hline parameters refined [restraints] & $343[0]$ & $447[0]$ \\
\hline GooF on $F^{2}$ & 1.096 & 1.041 \\
\hline$R$ indices $[F>4 \sigma(F)] R(F), w R\left(F^{2}\right)$ & $0.0228,0.0547$ & $0.0260,0.0508$ \\
\hline$R$ indices (all data) $R(F), w R\left(F^{2}\right)$ & $0.0264,0.0560$ & $0.0347,0.0533$ \\
\hline difference density: $\max , \min / \mathrm{e} \cdot \AA^{-3}$ & $0.977,-0.708$ & $0.525,-0.340$ \\
\hline
\end{tabular}

a numerical absorption correction. 
Table S5: Details of the crystal structure determinations of $\left[{ }^{\mathrm{Ph}} \mathrm{A}\right]-\mathbf{2}-\mathrm{Zr} \# \mathbf{1} \cdot \mathrm{C}_{6} \mathrm{D}_{6}$ and $\left[{ }^{\mathrm{Ph}} \mathrm{A}\right]-\mathbf{2}-\mathrm{Zr} \# \mathbf{2} \cdot$ toluene.

\begin{tabular}{|c|c|c|}
\hline compound & {$\left[{ }^{\mathrm{Ph}} \mathrm{A}\right]-2-\mathrm{Zr} \# 1 \cdot \mathrm{C}_{6} \mathrm{D}_{6}$} & {$\left[{ }^{\mathrm{Ph}} \mathrm{A}\right]-2-\mathrm{Zr} \# 2 \cdot$ toluene } \\
\hline formula & $\mathrm{C}_{38} \mathrm{H}_{27} \mathrm{D}_{6} \mathrm{I}_{2} \mathrm{~N}_{2} \mathrm{PZr}$ & $\mathrm{C}_{39} \mathrm{H}_{35} \mathrm{I}_{2} \mathrm{~N}_{2} \mathrm{PZr}$ \\
\hline$M_{\mathrm{r}}$ & 899.69 & 907.68 \\
\hline crystal system & monoclinic & monoclinic \\
\hline space group & $\mathrm{P} 22_{1} / c$ & $\mathrm{P} 2{ }_{1} / \mathrm{c}$ \\
\hline$a / \AA$ & $16.0851(2)$ & $16.0977(3)$ \\
\hline$b / \AA$ & $10.29017(12)$ & $10.43778(18)$ \\
\hline$c / \AA$ & $21.1915(2)$ & $21.0529(3)$ \\
\hline$\beta /^{\circ}$ & $96.7080(12)$ & $92.9851(13)$ \\
\hline$V / \AA^{3}$ & $3483.58(7)$ & $3532.61(10)$ \\
\hline$Z$ & 4 & 4 \\
\hline$F_{000}$ & 1744 & 1776 \\
\hline$d_{c} / \mathrm{Mg} \cdot \mathrm{m}^{-3}$ & 1.715 & 1.707 \\
\hline X-radiation, $\lambda / \AA$ & Mo- $K_{\alpha}, 0.71073$ & Mo- $K_{\alpha}, 0.71073$ \\
\hline$\mu / \mathrm{mm}^{-1}$ & 2.163 & 2.134 \\
\hline max., min. transmission factors & $0.973,0.899^{a}$ & $0.853,0.749^{a}$ \\
\hline data collect. temperature $/ \mathrm{K}$ & $120(1)$ & $120(1)$ \\
\hline$\theta$ range $/^{\circ}$ & $3.28-30.56$ & $3.30-32.33$ \\
\hline index ranges (indep. set) $h, k, l$ & $\pm 22, \pm 14, \pm 30$ & $-22 \ldots .24, \pm 15, \pm 31$ \\
\hline reflections measured & 104054 & 41109 \\
\hline unique $\left[R_{\text {int }}\right]$ & $10640[0.0705]$ & $11547[0.0426]$ \\
\hline observed $[I \geq 2 \sigma(I)]$ & 8761 & 9353 \\
\hline parameters refined [restraints] & $397[0]$ & $407[0]$ \\
\hline GooF on $F^{2}$ & 1.129 & 1.055 \\
\hline$R$ indices $[F>4 \sigma(F)] R(F), w R\left(F^{2}\right)$ & $0.0385,0.0668$ & $0.0359,0.0662$ \\
\hline$R$ indices (all data) $R(F), w R\left(F^{2}\right)$ & $0.0532,0.0705$ & $0.0503,0.0723$ \\
\hline difference density: $\max , \min / e \cdot \AA^{-3}$ & $1.224,-0.535$ & $0.835,-0.656$ \\
\hline
\end{tabular}

a numerical absorption correction. 
Table S6: Details of the crystal structure determinations of [B]-2-Zr and $\left[{ }^{\mathrm{Si}} \mathrm{A}\right]-\mathbf{2}-\mathrm{Zr}$.

\begin{tabular}{|c|c|c|}
\hline compound & {$[B]-2-Z r$} & {$\left[{ }^{\mathrm{Si}} \mathrm{A}\right]-2-\mathrm{Zr}$} \\
\hline formula & $\mathrm{C}_{36} \mathrm{H}_{35} \mathrm{I}_{2} \mathrm{~N}_{2} \mathrm{PZr}$ & $\mathrm{C}_{26} \mathrm{H}_{35} \mathrm{I}_{2} \mathrm{~N}_{2} \mathrm{PSi}_{2} \mathrm{Zr}$ \\
\hline$M_{\mathrm{r}}$ & 871.65 & 807.73 \\
\hline crystal system & monoclinic & monoclinic \\
\hline space group & $C 2 / c$ & $P 2_{1} / n$ \\
\hline$a / \AA$ & $21.784(11)$ & $11.15467(11)$ \\
\hline$b / \AA$ & $11.603(5)$ & $19.96395(19)$ \\
\hline$c / \AA$ & $27.779(13)$ & $14.66400(15)$ \\
\hline$\beta /^{\circ}$ & $90.962(7)$ & $102.0437(10)$ \\
\hline$V / \AA^{3}$ & $7020(6)$ & $3193.66(6)$ \\
\hline$Z$ & 8 & 4 \\
\hline$F_{000}$ & 3408 & 1576 \\
\hline$d_{c} / \mathrm{Mg} \cdot \mathrm{m}^{-3}$ & 1.649 & 1.680 \\
\hline X-radiation, $\lambda / \AA$ & Mo- $K_{\alpha}, 0.71073$ & Mo- $K_{\alpha}, 0.71073$ \\
\hline$\mu / \mathrm{mm}^{-1}$ & 2.144 & 2.420 \\
\hline max., min. transmission factors & $0.5655,0.5169^{a}$ & $0.761,0.634 \mathrm{~b}$ \\
\hline data collect. temperature $/ \mathrm{K}$ & $100(1)$ & $120(1)$ \\
\hline$\theta$ range $/^{\circ}$ & $1.99-30.51$ & $3.22-28.99$ \\
\hline index ranges (indep. set) $h, k, l$ & $\pm 31, \pm 16, \pm 39$ & $\pm 14, \pm 27, \pm 19$ \\
\hline reflections measured & 84962 & 132240 \\
\hline unique $\left[R_{\text {int }}\right]$ & $10735[0.0482]$ & $8204[0.0533]$ \\
\hline observed $[I \geq 2 \sigma(I)]$ & 8947 & 7583 \\
\hline parameters refined [restraints] & $383[0]$ & $313[0]$ \\
\hline GooF on $F^{2}$ & 1.050 & 1.211 \\
\hline$R$ indices $[F>4 \sigma(F)] R(F), w R\left(F^{2}\right)$ & $0.0300,0.0624$ & $0.0329,0.0625$ \\
\hline$R$ indices (all data) $R(F), w R\left(F^{2}\right)$ & $0.0417,0.0671$ & $0.0384,0.0642$ \\
\hline difference density: $\max , \min / \mathrm{e} \cdot \AA^{-3}$ & $2.235,-0.605$ & $0.808,-0.702$ \\
\hline
\end{tabular}

a empirical absorption correction. ${ }^{\mathrm{b}}$ numerical absorption correction. 
Table S7: Details of the crystal structure determinations of [B]-2-Hf·0.5 $\mathrm{Et}_{2} \mathrm{O}$ and $\left[{ }^{\mathrm{Ph}} \mathrm{A}\right]-\mathbf{3}-\mathrm{Zr}$.

\begin{tabular}{|c|c|c|}
\hline compound & {$[B]-2-H f \cdot 0.5 \mathrm{Et}_{2} \mathrm{O}$} & {$\left[{ }^{\mathrm{Ph}} \mathrm{A}\right]-3-\mathrm{Zr}$} \\
\hline formula & $\mathrm{C}_{38} \mathrm{H}_{40} \mathrm{Hfl}_{2} \mathrm{~N}_{2} \mathrm{O}_{0.5} \mathrm{P}$ & $\mathrm{C}_{40} \mathrm{H}_{49} \mathrm{~N}_{2} \mathrm{PSi}_{2} \mathrm{Zr}$ \\
\hline$M_{\mathrm{r}}$ & 995.98 & 736.18 \\
\hline crystal system & monoclinic & triclinic \\
\hline space group & $C 2 / c$ & $P-1$ \\
\hline$a / \AA$ & $22.0703(2)$ & $10.3850(2)$ \\
\hline$b / \AA$ & $11.52011(9)$ & $10.8151(3)$ \\
\hline$c / \AA$ & $29.7547(2)$ & $18.4097(5)$ \\
\hline$\alpha /^{\circ}$ & & $73.997(2)$ \\
\hline$\beta /{ }^{\circ}$ & $99.6396(9)$ & $89.0580(18)$ \\
\hline$\gamma /{ }^{\circ}$ & & $74.023(2)$ \\
\hline$V / \AA^{3}$ & $7458.36(11)$ & $1906.82(8)$ \\
\hline$Z$ & 8 & 2 \\
\hline$F_{000}$ & 3832 & 772 \\
\hline$d_{c} / \mathrm{Mg} \cdot \mathrm{m}^{-3}$ & 1.774 & 1.282 \\
\hline X-radiation, $\lambda / \AA$ & $\mathrm{Cu}-K_{\alpha}, 1.5418$ & $\mathrm{Cu}-K_{\alpha}, 1.54184$ \\
\hline$\mu / \mathrm{mm}^{-1}$ & 18.795 & 3.576 \\
\hline max., min. transmission factors & $0.0885,0.0151^{a}$ & $0.877,0.681^{b}$ \\
\hline data collect. temperature $/ \mathrm{K}$ & $120(1)$ & $120(1)$ \\
\hline$\theta$ range $/^{\circ}$ & $4.06-70.78$ & $4.43-70.86$ \\
\hline index ranges (indep. set) $h, k, l$ & $-26 \ldots 25, \pm 14, \pm 36$ & $\pm 12,-12 \ldots 13, \pm 22$ \\
\hline reflections measured & 105872 & 52674 \\
\hline unique $\left[R_{\text {int }}\right]$ & $7144[0.0355]$ & $7252[0.0617]$ \\
\hline observed $[I \geq 2 \sigma(I)]$ & 6926 & 6064 \\
\hline parameters refined [restraints] & $383[0]$ & $421[0]$ \\
\hline GooF on $F^{2}$ & 1.293 & 1.022 \\
\hline$R$ indices $[F>4 \sigma(F)] R(F), w R\left(F^{2}\right)$ & $0.0446,0.1093$ & $0.0326,0.0700$ \\
\hline$R$ indices (all data) $R(F), w R\left(F^{2}\right)$ & $0.0454,0.1101$ & $0.0456,0.0749$ \\
\hline difference density: $\max , \min / e \cdot \AA^{-3}$ & $2.165,-0.367$ & $0.674,-0.513$ \\
\hline
\end{tabular}

a empirical absorption correction. ${ }^{\mathrm{b}}$ numerical absorption correction. 
Table S8: Details of the crystal structure determinations of $\left[{ }^{\mathrm{Ph}} \mathrm{A}\right]-\mathbf{3}-\mathrm{Hf}$ and $\left[{ }^{\mathrm{Si}} \mathrm{A}\right]-\mathbf{4}-\mathrm{Hf}\left(\mathrm{C}_{1}\right) \cdot \mathrm{Et}_{2} \mathrm{O}$.

\begin{tabular}{|c|c|c|}
\hline compound & {$\left[{ }^{\mathrm{Ph}} \mathrm{A}\right]-3-\mathrm{Hf}$} & {$\left[{ }^{\mathrm{Si}} \mathrm{A}\right]-4-\mathrm{Hf}\left(C_{1}\right) \cdot \mathrm{Et}_{2} \mathrm{O}$} \\
\hline formula & $\mathrm{C}_{40} \mathrm{H}_{49} \mathrm{HfN}_{2} \mathrm{PSi}_{2}$ & $\mathrm{C}_{44} \mathrm{H}_{59} \mathrm{HfN}_{2} \mathrm{OPSi}_{2}$ \\
\hline$M_{\mathrm{r}}$ & 823.45 & 897.57 \\
\hline crystal system & triclinic & triclinic \\
\hline space group & $P-1$ & $P-1$ \\
\hline$a / \AA$ & $10.4057(3)$ & $14.1757(2)$ \\
\hline$b / \AA$ & $10.8811(4)$ & $16.0067(3)$ \\
\hline$c / \AA$ & $18.4072(5)$ & $20.6655(5)$ \\
\hline$\alpha /^{\circ}$ & $74.352(3)$ & $69.808(2)$ \\
\hline$\beta /{ }^{\circ}$ & $89.204(3)$ & $84.7664(16)$ \\
\hline$\gamma /{ }^{\circ}$ & $73.940(3)$ & $85.5702(15)$ \\
\hline$V / \AA^{3}$ & $1924.45(11)$ & $4377.34(16)$ \\
\hline$Z$ & 2 & 4 \\
\hline$F_{000}$ & 836 & 1840 \\
\hline$d_{c} / \mathrm{Mg} \cdot \mathrm{m}^{-3}$ & 1.421 & 1.362 \\
\hline $\mathrm{X}$-radiation, $\lambda / \AA$ & Mo- $K_{\alpha}, 0.71073$ & Mo- $K_{\alpha}, 0.71073$ \\
\hline$\mu / \mathrm{mm}^{-1}$ & 2.843 & 2.508 \\
\hline max., min. transmission factors & $0.844,0.734^{a}$ & $0.785,0.482^{a}$ \\
\hline data collect. temperature / $\mathrm{K}$ & $120(1)$ & $120(1)$ \\
\hline$\theta$ range $/^{\circ}$ & $3.26-26.37$ & $2.98-32.38$ \\
\hline index ranges (indep. set) $h, k, I$ & $\pm 13 \pm 13, \pm 22$ & $\pm 21,-23 \ldots 24, \pm 31$ \\
\hline reflections measured & 41030 & 194948 \\
\hline unique $\left[R_{\text {int }}\right]$ & $7837[0.0464]$ & $43447[0.055]$ \\
\hline observed $[I \geq 2 \sigma(I)]$ & 7427 & 31629 \\
\hline parameters refined [restraints] & $421[0]$ & $936[33]$ \\
\hline GooF on $F^{2}$ & 1.069 & 0.920 \\
\hline$R$ indices $[F>4 \sigma(F)] R(F), w R\left(F^{2}\right)$ & $0.0256,0.0612$ & $0.0345,0.0666$ \\
\hline$R$ indices (all data) $R(F), w R\left(F^{2}\right)$ & $0.0275,0.0624$ & $0.0542,0.0686$ \\
\hline difference density: $\max , \min / e \cdot \AA^{-3}$ & $2.788,-0.791$ & $2.986,-1.724$ \\
\hline
\end{tabular}

a numerical absorption correction. 
Table S9: Details of the crystal structure determinations of $\left[{ }^{\mathrm{Si}} \mathrm{A}\right]-4-\mathrm{Hf}\left(\boldsymbol{C}_{\mathrm{s}}\right)\left[{ }^{\mathrm{Ph}} \mathrm{A}\right]-\mathbf{- 4}-\mathrm{Zr} \cdot$ toluene.

\begin{tabular}{|c|c|c|}
\hline compound & {$\left[{ }^{S i} A\right]-4-H f\left(C_{s}\right)$} & {$\left[{ }^{\mathrm{Ph}} \mathrm{A}\right]-4-\mathrm{Zr}$-toluene } \\
\hline formula & $\mathrm{C}_{40} \mathrm{H}_{49} \mathrm{HfN}_{2} \mathrm{PSi}_{2}$ & $\mathrm{C}_{53} \mathrm{H}_{49} \mathrm{~N}_{2} \mathrm{PZr}$ \\
\hline$M_{\mathrm{r}}$ & 823.45 & 836.13 \\
\hline crystal system & monoclinic & triclinic \\
\hline space group & $P 2_{1} / n$ & $P-1$ \\
\hline$a / \AA$ & $10.25124(15)$ & $10.2300(3)$ \\
\hline$b / \AA$ & $39.7877(5)$ & $13.3848(3)$ \\
\hline$c / \AA$ & $10.27791(15)$ & $16.6135(3)$ \\
\hline$\alpha /^{\circ}$ & & $67.695(2)$ \\
\hline$\beta /{ }^{\circ}$ & $117.9006(19)$ & $86.916(2)$ \\
\hline$\gamma /{ }^{\circ}$ & & $86.495(2)$ \\
\hline$V / \AA^{3}$ & $3704.80(11)$ & 2099.53(9) \\
\hline$Z$ & 4 & 2 \\
\hline$F_{000}$ & 1672 & 872 \\
\hline$d_{c} / \mathrm{Mg} \cdot \mathrm{m}^{-3}$ & 1.476 & 1.323 \\
\hline $\mathrm{X}$-radiation, $\lambda / \AA$ & $\mathrm{Cu}-K_{\alpha}, 1.54184$ & Mo- $K_{\alpha}, 0.71073$ \\
\hline$\mu / \mathrm{mm}^{-1}$ & 6.456 & 0.339 \\
\hline max., min. transmission factors & $0.757,0.579^{a}$ & $0.974,0.948^{a}$ \\
\hline data collect. temperature / $\mathrm{K}$ & $120(1)$ & $120(1)$ \\
\hline$\theta$ range $/^{\circ}$ & $4.45-70.83$ & $3.05-30.54$ \\
\hline index ranges (indep. set) $h, k, I$ & $\pm 12,-44 \ldots 48, \pm 12$ & $\pm 14, \pm 19, \pm 23$ \\
\hline reflections measured & 94255 & 50443 \\
\hline unique $\left[R_{\text {int }}\right]$ & $7106[0.0311]$ & $12819[0.0591]$ \\
\hline observed $[I \geq 2 \sigma(I)]$ & 6989 & 10056 \\
\hline parameters refined [restraints] & $433[0]$ & $488[72]$ \\
\hline GooF on $F^{2}$ & 1.315 & 1.028 \\
\hline$R$ indices $[F>4 \sigma(F)] R(F), w R\left(F^{2}\right)$ & $0.0258,0.0487$ & $0.0419,0.0942$ \\
\hline$R$ indices (all data) $R(F), w R\left(F^{2}\right)$ & $0.0265,0.0489$ & $0.0603,0.1027$ \\
\hline difference density: $\max , \min / e \cdot \AA^{-3}$ & $0.545,-1.102$ & $0.748,-0.827$ \\
\hline
\end{tabular}

a numerical absorption correction. 
Table S10: Details of the crystal structure determinations of $\left[{ }^{\mathrm{Ph}} \mathrm{A}\right]-\mathbf{4}-\mathrm{Hf}$ and $[\mathrm{B}]-\mathbf{- 4}-\mathrm{Hf} \cdot$ solv.

\begin{tabular}{|c|c|c|}
\hline compound & {$\left[{ }^{\mathrm{Ph}} \mathrm{A}\right]-4-\mathrm{Hf}$} & [B]-4-Hf·solv \\
\hline formula & $\mathrm{C}_{46} \mathrm{H}_{41} \mathrm{HfN}_{2} \mathrm{P}$ & $\mathrm{C}_{50} \mathrm{H}_{49} \mathrm{~N}_{2} \mathrm{PHf}$ \\
\hline$M_{\mathrm{r}}$ & 831.27 & 887.37 \\
\hline crystal system & triclinic & monoclinic \\
\hline space group & $P-1$ & $\mathrm{C} 2 / \mathrm{c}$ \\
\hline$a / \AA ̊$ & $9.69030(19)$ & $24.105(13)$ \\
\hline$b / \AA$ & $13.2661(3)$ & $17.206(9)$ \\
\hline$c / \AA$ & $15.1478(4)$ & $22.372(11)$ \\
\hline$\alpha /^{\circ}$ & $100.832(2)$ & \\
\hline$\beta /^{\circ}$ & 92.9449(19) & $99.948(8)$ \\
\hline$\gamma /{ }^{\circ}$ & $101.4852(17)$ & \\
\hline$V / \AA^{3}$ & $1866.30(7)$ & $9140(8)$ \\
\hline$Z$ & 2 & 8 \\
\hline$F_{000}$ & 836 & 3600 \\
\hline$d_{c} / \mathrm{Mg} \cdot \mathrm{m}^{-3}$ & 1.479 & 1.290 \\
\hline $\mathrm{X}$-radiation, $\lambda / \AA$ & Mo- $K_{\alpha}, 0.71073$ & Mo- $K_{\alpha}, 0.71073$ \\
\hline$\mu / \mathrm{mm}^{-1}$ & 2.872 & 2.351 \\
\hline max., min. transmission factors & $0.899,0.747^{a}$ & $0.6478,0.5692^{b}$ \\
\hline data collect. temperature $/ \mathrm{K}$ & $120(1)$ & $100(1)$ \\
\hline$\theta$ range $/^{\circ}$ & $3.33-26.37$ & $1.65-29.18$ \\
\hline index ranges (indep. set) $h, k, l$ & $\pm 12, \pm 16, \pm 18$ & $\pm 32, \pm 23, \pm 30$ \\
\hline reflections measured & 41675 & 101635 \\
\hline unique $\left[R_{\text {int }}\right]$ & $7614[0.0520]$ & $12334[0.0838]$ \\
\hline observed $[I \geq 2 \sigma(I)]$ & 6883 & 9096 \\
\hline parameters refined [restraints] & $451[0]$ & $592[473]$ \\
\hline GooF on $F^{2}$ & 1.117 & 1.016 \\
\hline$R$ indices $[F>4 \sigma(F)] R(F), w R\left(F^{2}\right)$ & $0.0324,0.0843$ & $0.0410,0.0800$ \\
\hline$R$ indices (all data) $R(F), w R\left(F^{2}\right)$ & $0.0386,0.0868$ & $0.0658,0.0883$ \\
\hline difference density: $\max , \min / \mathrm{e} \cdot \AA^{-3}$ & $2.657,-0.857$ & $2.661,-2.409$ \\
\hline
\end{tabular}

${ }^{a}$ numerical absorption correction. ${ }^{\mathrm{b}}$ empirical absorption correction. 
Table S11: Details of the crystal structure determinations of [B]-5-Zr.pentane and [ $\left.{ }^{\mathrm{Ph}} \mathrm{A}\right]-\mathbf{6}-\mathrm{Zr}$.

\begin{tabular}{|c|c|c|}
\hline compound & [B]-5-Zr-pentane & {$\left[{ }^{\mathrm{Ph}} \mathrm{A}\right]-6-\mathrm{Zr}$} \\
\hline formula & $\mathrm{C}_{77} \mathrm{H}_{82} \mathrm{~N}_{4} \mathrm{P}_{2} \mathrm{Zr}$ & $\mathrm{C}_{64} \mathrm{H}_{59} \mathrm{~N}_{4} \mathrm{PZr}$ \\
\hline$M r$ & 1216.62 & 1006.34 \\
\hline crystal system & monoclinic & monoclinic \\
\hline space group & $P 2_{1} / n$ & $P 22_{1} / c$ \\
\hline$a / \AA$ & $20.2073(7)$ & $15.3382(3)$ \\
\hline$b / \AA$ & $14.3395(4)$ & $13.3600(3)$ \\
\hline$c / \AA$ & $23.1834(7)$ & $25.6716(5)$ \\
\hline$\beta /{ }^{\circ}$ & $107.080(4)$ & $104.950(2)$ \\
\hline$V / \AA^{3}$ & $6421.4(4)$ & $5082.51(19)$ \\
\hline$Z$ & 4 & 4 \\
\hline$F_{000}$ & 2568 & 2104 \\
\hline$d_{c} / \mathrm{Mg} \cdot \mathrm{m}^{-3}$ & 1.258 & 1.315 \\
\hline X-radiation, $\lambda / \AA$ & Mo- $K_{\alpha}, 0.71073$ & Mo- $K_{\alpha}, 0.71073$ \\
\hline$\mu / \mathrm{mm}^{-1}$ & 0.268 & 0.294 \\
\hline max., min. transmission factors & $0.986,0.954^{a}$ & $0.974,0.955^{a}$ \\
\hline data collect. temperature $/ \mathrm{K}$ & $120(1)$ & $120(1)$ \\
\hline$\theta$ range $/^{\circ}$ & $3.26-26.40$ & $3.35-27.53$ \\
\hline index ranges (indep. set) $h, k, I$ & $\pm 25, \pm 17, \pm 28$ & $\pm 19, \pm 17, \pm 33$ \\
\hline reflections measured & 141979 & 115679 \\
\hline unique $\left[R_{\text {int }}\right]$ & $13116[0.1074]$ & $11640[0.0920]$ \\
\hline observed $[I \geq 2 \sigma(I)]$ & 12267 & 9231 \\
\hline parameters refined [restraints] & $773[21]$ & $635[0]$ \\
\hline GooF on $F^{2}$ & 1.298 & 1.177 \\
\hline$R$ indices $[F>4 \sigma(F)] R(F), w R\left(F^{2}\right)$ & $0.0881,0.1478$ & $0.0673,0.1371$ \\
\hline$R$ indices (all data) $R(F), w R\left(F^{2}\right)$ & $0.0965,0.1514$ & $0.0877,0.1449$ \\
\hline difference density: $\max , \min / \mathrm{e} \cdot \AA^{-3}$ & $1.219,-0.990$ & $1.050,-0.916$ \\
\hline
\end{tabular}

a numerical absorption correction. 
Table S12: Details of the crystal structure determinations of [ $\left.{ }^{\mathrm{Si}} \mathrm{A}\right]-\mathbf{6}-\mathrm{Hf}$ and [B]-6-Hf.

\begin{tabular}{|c|c|c|}
\hline compound & {$\left[{ }^{\mathrm{Si}} \mathrm{A}\right]-6-\mathrm{Hf}$} & [B]-6-Hf \\
\hline formula & $\mathrm{C}_{49} \mathrm{H}_{58} \mathrm{HfN}_{3} \mathrm{PSi}_{2}$ & $\mathrm{C}_{70} \mathrm{H}_{72} \mathrm{~N}_{4} \mathrm{PO}_{0.5} \mathrm{Hf}$ \\
\hline$M_{\mathrm{r}}$ & 954.62 & 1186.77 \\
\hline crystal system & triclinic & triclinic \\
\hline space group & $P-1$ & $P-1$ \\
\hline$a / \AA$ & $11.21305(9)$ & $11.84756(13)$ \\
\hline$b / \AA$ & $17.74642(13)$ & $12.4209(2)$ \\
\hline$c / \AA$ & $22.97468(19)$ & $20.4206(3)$ \\
\hline$\alpha /^{\circ}$ & $98.1474(6)$ & $107.3504(15)$ \\
\hline$\beta /{ }^{\circ}$ & $91.2024(7)$ & $93.0867(11)$ \\
\hline$\gamma /{ }^{\circ}$ & $98.6327(6)$ & $95.0282(11)$ \\
\hline$V / \AA^{3}$ & $4470.18(6)$ & $2847.13(8)$ \\
\hline$Z$ & 4 & 2 \\
\hline$F_{000}$ & 1952 & 1222 \\
\hline$d_{c} / \mathrm{Mg} \cdot \mathrm{m}^{-3}$ & 1.418 & 1.384 \\
\hline X-radiation, $\lambda / \AA$ & $\mathrm{Cu}-K_{\alpha}, 1.54184$ & Mo- $K_{\alpha}, 0.71073$ \\
\hline$\mu / \mathrm{mm}^{-1}$ & 5.439 & 1.908 \\
\hline max., min. transmission factors & $0.766,0.555^{\mathrm{a}}$ & $0.949,0.788^{a}$ \\
\hline data collect. temperature $/ \mathrm{K}$ & $120(1)$ & $120(1)$ \\
\hline$\theta$ range $/^{\circ}$ & $3.42-70.86$ & $3.29-32.39$ \\
\hline index ranges (indep. set) $h, k, l$ & $\pm 13, \pm 21,-27 \ldots 28$ & $\pm 18, \pm 18, \pm 30$ \\
\hline reflections measured & 243784 & 84075 \\
\hline unique $\left[R_{\text {int }}\right]$ & $17078[0.0298]$ & $19278[0.0391]$ \\
\hline observed $[I \geq 2 \sigma(I)]$ & 16751 & 17967 \\
\hline parameters refined [restraints] & $1025[0]$ & $738[23]$ \\
\hline GooF on $F^{2}$ & 1.137 & 1.086 \\
\hline$R$ indices $[F>4 \sigma(F)] R(F), w R\left(F^{2}\right)$ & $0.0261,0.0565$ & $0.0280,0.0575$ \\
\hline$R$ indices (all data) $R(F), w R\left(F^{2}\right)$ & $0.0267,0.0568$ & $0.0319,0.0589$ \\
\hline difference density: $\max , \min / e \cdot \AA^{-3}$ & $1.119,-1.498$ & $1.017,-1.091$ \\
\hline
\end{tabular}

a numerical absorption correction. 


\section{X-Ray Crystal Structure Determinations}

Crystal data and details of the structure determinations are compiled in Tables S1 - S12. Full shells of intensity data were collected at low temperature with a Bruker AXS Smart 1000 CCD diffractometer (Mo$K_{\alpha}$ radiation, sealed $X$-ray tube, graphite monochromator; compounds $[\mathrm{B}] \mathrm{H}_{2},\left[{ }^{\mathrm{Ph}} \mathrm{A}\right]-\mathbf{1}-\mathbf{M}(\mathrm{M}=\mathrm{Ti}, \mathrm{Zr}),[\mathrm{B}]-$ 1-Hf, [B]-2-Zr and [B]-4-Hf) or an Agilent Technologies Supernova-E CCD diffractometer (Mo- or Cu- $K_{\alpha}$ radiation, microfocus $\mathrm{X}$-ray tube, multilayer mirror optics; all other compounds). Detector frames (typically $\omega$-, occasionally $\varphi$-scans, of $0.4 \ldots 1^{\circ}$ ) were integrated by profile fitting. ${ }^{4}$ Data were corrected for air and detector absorption, Lorentz and polarization effects ${ }^{5,6}$ and scaled essentially by application of appropriate spherical harmonic functions. ${ }^{7,8}$ Absorption by the crystal was treated with a semiempirical multiscan method (as part of the scaling process) and augmented by a spherical correction, ${ }^{7,8,9,}$, numerically (Gaussian grid). ${ }^{6,10}$ For datasets collected with the microfocus tube(s) an illumination correction was performed. ${ }^{8}$

The structures were solved by intrinsic phasing (complexes $\left[{ }^{\mathrm{Si}} \mathrm{A}\right]-\mathbf{4}-\mathbf{H f}$ and $[\mathrm{B}]-\mathbf{5}-\mathbf{Z} \mathbf{r}^{11}$ ) or by the charge flip procedure (all other structures) ${ }^{12}$ and refined by full-matrix least squares methods based on $F^{2}$ against all unique reflections. ${ }^{13}$ All non-hydrogen atoms were given anisotropic displacement parameters. Hydrogen atoms were generally input at calculated positions and refined with a riding model. When justified by the quality of the data the positions of some hydrogen atoms were taken from difference Fourier syntheses and refined. When found necessary, disordered groups and/or solvent molecules were subjected to suitable geometry and adp restraints or constraints. Due to severe disorder and/or fractional occupancy, electron density attributed to solvent of crystallization was removed from the structures of [B]-1-Hf (diethyl ether), [B]-1-Ti (diethyl ether or pentane), [B]-2-Hf (diethyl ether), [B]-4-Hf (diethyl ether or pentane) and [ $\left.{ }^{\mathrm{Ph}} \mathbf{A}\right]-\mathbf{4}-\mathbf{Z r}$ (toluene) with the BYPASS procedure, ${ }^{14}$ as implemented in PLATON (SQUEEZE). ${ }^{15}$ Partial structure factors from the solvent masks were included in the refinement as separate contributions to $F_{\text {calc. }}$ Crystals of $\left[{ }^{\mathrm{Si}} \mathrm{A}\right]-4-\mathrm{Hf}$, mounted at around $-50^{\circ} \mathrm{C}$, underwent a phase transition on the diffractometer at $120 \mathrm{~K}$ within minutes to give the twinned structure described in this work. This structure was refined against all singles and composites involving both domains (refined twin fractions 0.4913(3):0.5087) 


\section{5) References}

(1) Suzuki, T.; Imai, K.; Nakagawa, H.; Miyata, N., ChemMedChem 2006, 1, 1059.

(2) Lehmann, J.; Kraft, G., Arch. Pharm. 1982, 315, 967.

(3) Batke, S.; Sietzen, M.; Wadepohl, H.; Ballmann, J., Inorg. Chem. 2014, 53, 4144.

(4) Kabsch, K. in: Rossmann, M. G., Arnold, E. (eds.), "International Tables for Crystallography" Vol. F, Ch. 11.3, Kluwer Academic Publishers, Dordrecht, 2001.

(5) SAINT, Bruker AXS GmbH, Karlsruhe, Germany 1997-2013.

(6) CrysAlisPro, Agilent Technologies UK Ltd., Oxford, UK 2011-2014 and Rigaku Oxford Diffraction, Rigaku Polska Sp.z o.o., Wrocław, Poland 2015-2016.

(7) (a) Sheldrick, G. M., SADABS, Bruker AXS GmbH, Karlsruhe, Germany 2004-2014; (b) Krause, L; Herbst-Irmer, R.; Sheldrick, G. M.; Stalke, D., J. Appl. Cryst. 2015, 48, 3; (c) Sheldrick, G. M., TWINABS, Bruker AXS GmbH, Karlsruhe, Germany 2004-2012.

(8) SCALE3 ABSPACK, CrysAlisPro, Agilent Technologies UK Ltd., Oxford, UK 2011-2014 and Rigaku Oxford Diffraction, Rigaku Polska Sp.z o.o., Wrocław, Poland 2015-2016.

(9) Blessing, R. H., Acta Cryst. 1995, A51, 33.

(10) Busing, W. R.; Levy, H. A., Acta Cryst. 1957, 10, 180.

(11) (a) Sheldrick, G. M., SHELXT, University of Göttingen and Bruker AXS GmbH, Karlsruhe, Germany, 2012-2014; (b) Ruf, M; Noll, B. C., Application Note SC-XRD 503, Bruker AXS GmbH Karlsruhe, Germany 2014; (c) Sheldrick, G. M., Acta Cryst. 2015, A71, 3.

(12) (a) Palatinus, L., SUPERFLIP, EPF Lausanne, Switzerland and Fyzikální ústav AV ČR, v. v. i., Prague, Czech Republic, 2007-2014 (b) Palatinus, L.; Chapuis, G., J. Appl. Cryst. 2007, 40, 786.

(13) (a) Sheldrick, G. M., SHELXL-20xx, University of Göttingen and Bruker AXS GmbH, Karlsruhe, Germany 2012-2014; (b) Sheldrick, G. M., Acta Cryst. 2008, A64, 112; (c) Sheldrick, G. M., Acta Cryst. 2015, C71, 3.

(14) (a) v. d. Sluis, P.; Spek, A. L. Acta Cryst. 1990, A46, 194; (b) Spek, A. L. Acta Cryst. 2015, C71, 9.

(15) (a) Spek, A. L. PLATON, Utrecht University, The Netherlands; http://www.platonsoft.nl; (b) Spek, A. L. J. Appl. Cryst. 2003, 36, 7. 